\title{
ANISOTROPY OF THIN-WALLED TUBES BY A NEW METHOD OF COMBINED TENSION AND SHEAR LOADING
}

\author{
by \\ Christopher P. Dick and Yannis P. Korkolis* \\ *Corresponding author. Tel:+1-603-862-2772, e-mail: yannis.korkolis@unh.edu
}

Submitted - September 2014

Revised - February 2015

$2^{\text {nd }}$ Revision - April 2015

Department of Mechanical Engineering

University of New Hampshire

33 Academic Way, Durham, NH, 03824, USA 


\section{Abstract:}

We propose an experimental method for generating combined tension and shear stressstates in thin-walled tubes, with the purpose of calibrating anisotropic yield functions. The method involves extracting wide rings from the parent tubes and machining two notches that form the test-section between them. The rings are then fitted over two semicircular, or D-shaped, mandrels that are parted in a universal testing machine. The test-section remains on the top mandrel and, by virtue of its geometry, it experiences plane-strain deformation during this stretching. Hence we term the specimens as Ring Plane-Strain Tension specimens, or RPST. By controlling the orientation of the notches and thus the test-section, infinite combinations of tension and shear on the test-section can be created. We begin by describing the design of a family of RPST specimens using numerical simulation and comparing them to existing similar designs for sheets. We then proceed to describe RPST experiments that we performed on extruded Al6061-T4 tubes. We use Digital Image Correlation to probe the strain fields in the testsection and establish the validity of the plane-strain conditions. Subsequently, we describe the post-processing method that we propose for the RPST specimens. Finally, we calibrate two non-quadratic anisotropic yield functions to the data. We demonstrate the value of the proposed method and highlight that due to its simplicity, it can be used in conjunction with existing experimental methods to generate a more complete picture of the material anisotropy.

Keywords: Yield condition; anisotropic material; constitutive behavior; mechanical testing. 


\section{INTRODUCTION}

The accuracy of predictions of plastic flow and failure during material forming processes depends on the anisotropic yield function that is used for representing the material behavior (e.g., Kuwabara, 2007, Kuwabara et al., 2011, Korkolis and Kyriakides, 2011a, 2011b). This is because the local normals to the yield surface determine the direction of plastic flow and hence the strains induced for a given increment in the load. Without accurate predictions of the strain paths, predictions of failure can be expected to suffer, as well. For these reasons, a large number of anisotropic yield functions have been proposed to-date (Soare et al., 2008, Banabic, 2009, Aretz and Barlat, 2013, Yoshida et al., 2013, among others). The trend has been towards functions that are flexible enough to capture the shape of the yield locus, usually at the expense of having many parameters that need to be calibrated.

In the case of thin-walled tubes, the methods that have essentially evolved for calibrating anisotropic yield functions are suited to the tubular geometry of the starting material, and thus are distinct from the more established methods for sheets. These oftentimes involve testing of the tubes under axial load and torsion, or axial load and internal pressure. While these methods are flexible in prescribing the loading path, they require specialized equipment and are oftentimes cumbersome to perform. Here we propose a method that can generate multiaxial data from tube testing, using minimal experimental equipment. In addition, the method can generate experimental data that are complementary to, rather than competing with some of the existing techniques.

In our previous work (Dick \& Korkolis, 2014a, b), we investigated the mechanics of the Ring Hoop Tension Test, shown in Fig. 1a. In this experiment, a test-section that 
resembles a standard tensile specimen is machined on a ring extracted from the extruded Al-6061-T4 tubes under investigation. This ring is placed over two closelyfitting, semicircular or D-shaped mandrels, which are then parted with the help of a universal testing machine. Since the test-section of the ring specimen remains in contact with the top mandrel throughout the test, its curvature remains constant and hence it experiences stretching only. With the benefit of full-field Digital Image Correlation (DIC) and the use of Finite Element Analysis (FEA), we quantified and accounted for the effects of contact-induced stresses, specimen-mandrel friction and wall-thickness eccentricity of the starting specimen on the recorded responses. Ultimately, by decoupling these effects we determined the material response in the hoop direction of the tube. By comparing it to the axial response, the anisotropy of the Al-6061-T4 tube between these two directions was established (see Fig. 1b). In addition, the use of DIC allowed for the determination of the plastic strain ratios (or Rvalues) throughout the experiment. These ratios established the anisotropy between the axial and hoop responses very clearly and where in accord with the variation in the material responses shown in Fig. 1b (see Dick \& Korkolis, 2014a for details). However, to be able to calibrate a suitable anisotropic yield function, the material response along more directions is needed. This motivated the present study, in which we propose and investigate a family of specimen geometries that generate multiple loading paths under combined tension and shear. This is accomplished by replacing the tensile-like specimen shown in Fig. 1a with specimens that generate plane-strain stress-states (as proposed already by Link et al., 1998) and then rotating the test-section with respect to 
the tube generatrix to varying degrees, thus inducing in it a combination of tensile and shear stresses.

We begin this paper by describing the design of this specimen family, which we will be calling Ring Plane-Strain Tension, or RPST, specimens. We use FEA to assess the performance of the proposed geometries, and compare them to an established planestrain tension specimen design for sheets. We then describe the RPST experiments that we performed on extruded Al-6061-T4 tubes, including the use of 3D DIC. We describe in detail a particular experiment as representative of the entire population. In addition, with the aid of the DIC we verify the plane-strain conditions that the specimens are designed to produce. We then propose a post-processing procedure to account for the effects of the net force acting on the oddly oriented test-section and of the nonuniformity of the stress fields in the test-section. Finally, we present our results on the anisotropy of the Al-6061-T4 tubes and calibrate two popular anisotropic material models (one plane-stress and one fully 3D) that can be used in numerical simulations of the tube response during forming.

\section{SPECIMEN DESIGN FOR RING PLANE-STRAIN TENSION}

\section{a. Overview}

The Ring Plane-Strain Tension (RPST) specimen that we propose is shown in Fig. 2. The design is directly influenced by the variety of plane-strain tension specimen designs in the literature that are extracted from sheets, as for example shown in Fig. 3 from Tardif and Kyriakides, (2012). In the sheet designs, the test-section (the speckled region in the center of the specimen in Fig. 3) is formed by cutting two notches of 
suitable geometry in the sheet. Hence the test-section is confined between two wide shoulders (or ears) that are designed to remain elastic during loading. In this way, the strain that is lateral to the direction of the load (termed, "strain parallel to the notches" in this work) is minimal in the ears, as it corresponds to the elastic Poisson's strain. This stiff material acts as a constraint to the plastically deforming test-section, with the result that the parallel strain inside the test-section is minimal, as well. Hence, conditions that are close to plane-strain prevail inside the test-section. Naturally, the two free sides of the test-section are in a state of uniaxial tension, which then transitions to plane-strain as it moves to the center of the test-section. The determination of the stress-state inside the test-section is a major component of the present work and is described in Sections 4 and 5.

A note to clarify the terminology of the paper: all directions that are discussed are "in-plane", i.e., they lie on the surface of the tube. No reference to the radial, or "out-ofplane", direction is implied unless explicitly stated. The terms "perpendicular" and "parallel" refer to cross-sectional planes with a normal vector that is in-plane and is oriented in one of these two directions with respect to the two notches. The terms "normal" and "shear" are used for traction vectors and stress components, as usual. Hence the terms "perpendicular normal stress" and "perpendicular shear stress", where the first word identifies the plane and the second the direction of the traction vector.

\section{b. Detailed specimen design}

An important ratio to maximize when designing an effective plane-strain specimen is the width-to-height ratio of the test-section (i.e., width across and height of the test- 
section shown as the speckled area in Fig. 3). Ideally, of course, the width should be infinite, for plane-strain conditions to prevail. At the same time, in our work the width could not be greater than the $25.4 \mathrm{~mm}$ opening of the clevis grips used to load the specimen, as the plastic deformation would then occur outside of the test-section. Then, for a finite width, the height of the test-section should be as small as possible. On the other hand, this height cannot be reduced below a minimum that can be accurately measured with confidence by the DIC system. Furthermore, as the test-section height is reduced, the stress concentration at the root of the notches is intensified, leading to less favorable conditions for plane-strain deformation to occur over a sizeable portion of the test-section.

The shape of the two notches was arrived at after suitable parametric studies, as well as comparison to other, competing geometries (Dick, 2014). The notch opening was $3.175 \mathrm{~mm}$. We opted for a straight edge of the test-section that meets the flanks of each notch with a $0.794 \mathrm{~mm}$ (or $1 / 32$ inch.) radius. This presented the best compromise between a perfectly sharp corner, which could increase the useful height of the testsection for DIC purposes but introduce a high stress concentration and potentially lead to an early failure, and the machining convenience of using a radius equal to half of the notch opening. The final dimensions of the test-section were $20 \mathrm{~mm}$ (width) by 3.175 $\mathrm{mm}$ (height). The width of the RPST specimen at the region of the ears was set to 50 $\mathrm{mm}$. An engineering drawing of the proposed specimen is presented in the Appendix. 


\section{c. Performance assessment of the specimen}

The performance of the proposed RPST specimen was determined by comparing the uniformity of the stress and strain fields in the test-section to those of the sheet plane-strain tension specimen proposed by Tardif and Kyriakides (2013).

A finite element model of the RPST specimen was created in the non-linear implicit code Abaqus/Standard. Taking advantage of the symmetries present, only half of the specimen was simulated (see Fig. 4a). The model was meshed with five solid, linear, reduced integration elements (C3D8R) through the thickness. This mesh arrangement was shown to be sufficient in our earlier work (Dick, 2014, Dick and Korkolis, 2014a). The use of linear elements was necessary due to the presence of contact. The Dshaped mandrels (or D-blocks) were represented by analytical rigid shells, and surfaceto-surface contact with a no-penetration normal behavior ("hard" contact) was used. A low Coulomb friction value of $\mu=0.01$ was chosen, to keep the frictional effects negligible. The material model adopted for this study was the rate-independent $\mathrm{J}_{2}$ flow theory of plasticity with isotropic hardening and an associated flow rule. The hardening curve of the Al-6061-T4 material adopted was determined experimentally in (Dick and Korkolis, 2014a, b). (The engineering stress-strain version of this curve is identified in Fig. 1b as "Axial sts-stn").

The simulation was run in two steps. In the first step, the small initial gap between the D-shaped mandrels and the ring was closed by displacing both mandrels vertically. In the second step, the top mandrel was held in place while the bottom mandrel was displaced vertically downwards, replicating the operation of the testing machine used for the experiments. Note that the test-section rests on the top mandrel throughout the 
loading, avoiding the expanding gap between the separating mandrels and the associated bending effects.

The true strain parallel to the notches $\left(\varepsilon_{\square}\right)$ and true stress perpendicular to them $\left(\sigma_{\perp}\right)$ were output from the integration points of the surface layer of elements across the center of the test-section and compared to the corresponding distributions from the sheet plane-strain tension specimen of Fig. 3. The results are shown in Figs. 4b and c. For both metrics, the two specimens behave in a very similar manner. The parallel true strain $\left(\mathcal{E}_{\square}\right)$ is almost zero in the central $60 \%$ of the test-section width, verifying the planestrain conditions. Interestingly, the perpendicular true stress $\left(\sigma_{\perp}\right)$ is seen to continuously vary along the width of the test-section, i.e., it does not exhibit a plateau in the same sense as $\varepsilon_{\square}$ does.

\section{d. Additional specimen geometries}

The results of the previous section indicate that a plane-strain tension specimen can be produced for a ring geometry, by a straightforward adaptation of existing designs for sheets. However, such a specimen would yield only one additional stress-state useful for calibrating an anisotropic yield function. To increase the number of attainable stressstates, additional RPST specimens were designed by rotating the test-section with respect to the tube generatrix. Therefore, the test-section is now extending along a helical arc that wraps on the circular-cylindrical surface of the top D-shaped mandrel. Three additional RPST specimen designs were created, with notch angles of $15^{\circ}, 30^{\circ}$ and $45^{\circ}$, as shown in Fig. 5. The same notch and test-section dimensions from the $0^{\circ}$ RPST specimen were used. 
It can be expected that as these specimens are placed on the two D-shaped mandrels and tested, they will develop not only stretching but shearing, as well. Moreover, the ratio between the two modes will be controlled by the rotation of the testsection away from the generatrix, allowing us to generate multiple, well-controlled stress-states.

The performance of these specimens was assessed in the same fashion as described above in Section $2 \mathrm{c}$ for the $0^{\circ}$ RPST specimen. The results for the parallel true strain $\left(\varepsilon_{\square}\right)$ along each test-section width are plotted for the four RPST specimens $\left(0^{\circ}, 15^{\circ}, 30^{\circ}\right.$ and $\left.45^{\circ}\right)$ in Fig. 6. (As shown in Fig. 5, each specimen has its own coordinate system that is parallel and perpendicular to the test-section between the notches. In the case of the $15^{\circ}, 30^{\circ}$ and $45^{\circ}$ specimens, this coordinate system follows the helix that the test-section is forming.) It is interesting to note that increasing the rotation away from the generatrix does not negatively affect the performance of the specimen with regards to the plane-strain assumption in the central $60 \%$ of the testsection width. In addition, the magnitude of the strain outside of that central region is lessened, as the rotation angle increases.

The wrapping of the test-section along a helical arc introduces the possibility of the results being affected by the specimen-mandrel friction, as was the case with the Ring Hoop Tension Test examined in our previous work (Dick and Korkolis, 2014a, b). This was examined by selecting the $30^{\circ}$ RPST specimen and re-running the simulations with higher coefficients of friction. As shown in Fig. 7, Coulomb friction coefficients up to 0.1 (which is what we determined experimentally to be the actual friction coefficient between the specimen and the mandrel in our previous work, Dick and Korkolis 2014a) 
have no noticeable effect on the results. This is perhaps expected because the structural confinement that is offered by the ears has greater impact on the stress-state inside the test-section than does the variation due to friction within this narrow zone.

\section{RING PLANE STRAIN TENSION EXPERIMENTS}

\section{a. Experimental set-up}

The four Ring Plane Strain Tension specimens $\left(0^{\circ}, 15^{\circ}, 30^{\circ}, 45^{\circ}\right)$ designed through the use of FEA were prepared from extruded Al-6061-T4 tubes. The tubes had an outside diameter of $60 \mathrm{~mm}$ and nominal wall-thickness of $3 \mathrm{~mm}$. The tube material was characterized extensively in our previous work (see Dick and Korkolis, 2014a, b). As described there, the tubes were extruded through a porthole die and thus had 3 cold welds running the length of them. In the present work, care was taken such that all testsections were located in the base material and came from the same circumferential location along the tubes.

To prepare the specimens, rings of $50 \mathrm{~mm}$ width were parted from the Al-6061-T4 tubes using a lathe. No attempt was made to correct the wall-thickness eccentricity of the parent tubes by turning them to a uniform wall-thickness, as this was found to affect to some degree the material response (Dick and Korkolis, 2014a). The notches were then machined using a conventional vertical milling machine. Finally, the reduced sections at the top and bottom of the specimen for clevis grip clearance (see Fig. 1a and 2) were machined in the same machine.

The specimens were tested on a $250 \mathrm{kN}$ servohydraulic testing machine (MTS Landmark 370 with a Flextest 40 controller and the MTS 793 data acquisition and 
control software), equipped with hydraulic wedge grips (model MTS 647). The same Dshaped mandrels and clevis grips as in our previous work were used. The experiments were performed under displacement control, with the velocity of the actuator set to 25.4 $\times 10^{-3} \mathrm{~mm} / \mathrm{s}$ to correspond to quasistatic testing. The three-dimensional Digital Image Correlation system VIC-3D from Correlated Solutions, Inc., was used to capture the full strain fields during deformation and verify that the specimens achieve a plane-strain condition. The use of 3D DIC was necessary due to the curvature of the specimens. Two 2.0 Megapixel digital cameras (Point Grey Research, Inc.) with $35 \mathrm{~mm}$ Schneider lenses and the VIC-Snap software were used for acquiring the images during the tests.

\section{b. Description of a typical experiment}

A specimen mounted on the testing machine is shown in Fig. 8. This figure also shows how the test-section between the notches rests on the top D-shaped mandrel to avoid the expanding gap between the two mandrels and the associated bending effects. Also, the speckle pattern extends beyond the test-section to allow probing of the strainstate in the ears, as needed.

The load-displacement data collected by the testing machine's load cell and displacement transducer for each RPST specimen is shown in Fig. 9. The $0^{\circ}$ and $15^{\circ}$ specimens (brown and red curves) experienced a somewhat sudden failure during testing, with rapid growth of the strain localization and rupture. This is indicated in Fig. 9 by the sharp drop in their load-displacement curves. In contrast, the $30^{\circ}$ and $45^{\circ}$ specimens (blue and green curves) experienced a more gradual failure. In these specimens, the rupture initiated at one of the two radii of the top notch and propagated 
somewhat slowly across the test-section to the bottom notch. This event is indicated by the jagged tails in the corresponding load-displacement curves shown in Fig. 9.

To further investigate the onset and gradual propagation of the rupture noticed in the $30^{\circ}$ and $45^{\circ}$ specimens, a closer look at the behavior of the $30^{\circ}$ specimen is shown in Figs. 10 and 11. Six points along the load-displacement curve, labelled ' $a$ '- $f$ ' in Fig. 10, were chosen to investigate the specimen deformation. These points correspond to, ' $a$ ' small straining, 'b' - plane-strain deformation, 'c' - deformation beyond the realm of plane-strain, 'd' - onset of rupture, 'e' - propagation of rupture, 'f' - final separation. The images of this progression as captured by one of the two DIC cameras are shown in Fig. 11. In Fig. 11a, the deformation of the test-section is very small, so the specimen looks very similar to an untested one. In Fig. 11b, the test-section is beginning to experience more deformation; however the specimen test-section is still in a state of plane-strain, as will be shown subsequently from inspection of the DIC strain fields. Note the slight distortion in the top notch between instances ' $a$ ' and 'b'. In Fig. 11c, the specimen has deformed beyond the realm of plane-strain (i.e., the DIC strain fields that are discussed subsequently reveal that the strain parallel to the notch, $\mathcal{E}_{\square}$, is no longer close enough to zero for a plane-strain condition to exist). Again note the increasing distortion of the top notch between instances 'b' and 'c'. In Fig. 11d, a crack has formed at the top notch and the rupture has begun to propagate along the test-section of the specimen. The onset and propagation of the rupture reduces the load-bearing capacity of the specimen, indicated by the dropping load in Fig. 10. This rupture has propagated half-way across the test-section in Fig. 11e, and it has caused the test-section to fully separate in Fig. $11 \mathrm{f}$. 


\section{c. Full strain field investigation using DIC}

The true strain tensor at the center of the specimen test-section was monitored throughout the simulation. Once the true stresses are determined as discussed in Section 4, the incremental plastic work can be found as:

$$
d W^{p}=\sigma_{i j} d \varepsilon^{p}{ }_{i j}
$$

which in the present case can be expanded to read:

$$
d W^{p}=\sigma_{\perp} d \epsilon_{\perp}^{p}+\sigma_{\|} d \epsilon_{\|}^{p}+2 \tau d \epsilon_{\perp \|}^{p}
$$

(Note that with this term we are essentially referring to "plastic work density".) Recall that each specimen has its own coordinate system aligned with its notch angle, as shown in Fig. 5, so that for every specimen the symbols " $\perp$ " and " $\square$ " indicate quantities in the perpendicular and parallel to each test-section directions, respectively.

The parallel engineering strain output $\left(\varepsilon_{\square}\right)$ from the 3D DIC of each specimen was probed and is shown in Fig. 12 at an instance of equal plastic work (3 MPa) in the center of each specimen. The DIC contours in Fig. 12 show that at the center of each specimen the parallel strain $\mathcal{E}_{\square}$ is very low, verifying the FEA predictions of a planestrain condition in that location.

To further substantiate that the plane-strain condition exists, the parallel and perpendicular true strains across the notch were systematically probed at increasing levels of plastic work. Figures 13 and 14 a show the results of this probing for a $0^{\circ}$ and a $45^{\circ}$ RPST specimen (the two extreme cases), respectively, while Fig. 14b shows the shear strain distribution for the $45^{\circ}$ specimen. Each colored line corresponds to an 
instance of deformation when the output was extracted. In these figures, the x-axis is the normalized distance across the test-section. In Figs. 13 and 14a, the positive $y$-axis is the perpendicular true strain $\left(\varepsilon_{\perp}\right)$ and the negative $y$-axis is the parallel true strain $\left(\mathcal{E}_{\square}\right)$. Comparing these two strains indicates that the material in the central region of the test-section is deforming perpendicularly to the cross-section between the notches much faster than it does in the parallel direction. In addition, it can be seen that the parallel true strain remains approximately zero for a significant portion of the loading, confirming the plane-strain condition. Also, by comparing Figs. 13 and 14a, it is noticed that the $45^{\circ}$ specimen's parallel strain grows much slower than that of the $0^{\circ}$ specimen, indicating that the $45^{\circ}$ specimen holds the plane-strain condition to a higher level of plastic work. Moving on to Fig. 14b, the $y$-axis is the shear strain of the $45^{\circ}$ RPST specimen at the same levels of plastic work as in Figs. 13 and 14a. As shown in Fig. $14 \mathrm{~b}$, the shear strain $\left(\varepsilon_{\perp \square}\right)$ is greater than the perpendicular strain $\left(\varepsilon_{\perp}\right)$ for the $45^{\circ}$ specimen (Fig. 14a) at each level of plastic work. It is apparent that the presence of shear does not affect the plane-strain conditions inside the test-section.

\section{d. Closing remarks on observations of failure}

The purpose of the RPST specimens designed is to aid in the identification of anisotropy of a material, and not to probe failure. This is indicated from the immediately preceding discussion of Section 3c, where the DIC data show that the strain-state in every specimen deteriorates uncontrollably at the advanced stages of deformation before failure. A failed $0^{\circ}$ specimen is shown in Fig. 15. The DIC paint has been removed from around the test-section, to visualize the rupture more easily. There is 
significant deformation-induced roughening around the test-section. The final rupture is at an angle to the free surface of the test-section. As described in Section 3b, failure of that specimen was sudden and its evolution was not captured by the DIC cameras due to an incompatible sampling rate. However, judging from the response of the $30^{\circ}$ and $45^{\circ}$ specimens, the rupture must have initiated from one of the fillets shown in Fig. 15 and propagated across the test-section. Instead, with the anisotropy of the material established with the proposed RPST specimens a different specimen design, tailored to probing the ductile fracture could be used, as in the approach by (Mohr et al., 2010, Stoughton and Yoon, 2011, Dunand et al., 2012, Luo et al., 2012).

\section{POST-PROCESSING OF THE RING PLANE STRAIN TENSION EXPERIMENTS}

\section{a. Overview}

The RPST specimens present two unique post-processing challenges, in comparison to our earlier work on the Ring Hoop Tension Test (or RHTT, Dick and Korkolis, 2014a). First, due to the more complex geometry, lack of symmetry and spatial orientation, the force that is transmitted through the test-section is not simply half of the load cell recording, as was the case in RHTT. Second, as discussed earlier in Section $2 \mathrm{c}$, the true stress profile across the test-section of the RPST specimens is non-uniform (see Fig. 4c), due to the transition from uniaxial tension at the free edges of the testsection to plane-strain tension at the central region. This non-uniformity causes an error in calculating the perpendicular normal stress $\left(\sigma_{\perp}\right)$ and shear stress $(\tau)$ in the testsection using a simple "force/area" approach. Since the strain information is extracted from the central region of the test-section, where plane-strain conditions prevail (see 
Figs. 13 and 14), this inconsistency between stress and strain creates an error in calculating the incremental plastic work $\left(d W^{p}\right)$. The magnitude of this error can be expected to be different among the different specimen geometries, which will artificially distort the experimental yield locus at any given level of plastic work.

To account for these two errors, the appropriate correlation between the load cell reading and the load on the test-section, as well as a correction of the average stress (i.e., Force/Area) to account for the stress non-uniformity are discussed in this Section. The entire post-processing procedure is presented schematically as a flowchart in Fig. 16.

\section{b. Post-processing approach}

\section{b1. Correction for the force orientation}

The first step in determining the correct stress-state in the test-section is to determine the total load that the test-section carries. The component of this load that is perpendicular to the test-section (referred to as the perpendicular force $F_{\perp}$ ) is determined with the help of the FEA model described in Section 2c. (Recall that this model uses the von Mises yield function and the hardening curve of Al-6061-T4 that was determined in Dick and Korkolis, 2014a.) This is done by selecting a cross-section inside the test-section and multiplying at each increment of the simulation the perpendicular true stress $\left(\sigma_{\perp}\right)$ by the current cross-sectional area, both available in the FEA model. The reaction force on the top D-block in the FEA model is also monitored during the simulation and corresponds to the load cell reading, $F_{L C}$, in the experiment. The ratio between the total load cell reading $F_{L C}$ and the perpendicular force $F_{\perp}$, termed 
the perpendicular force coefficient $\alpha_{\perp}$, can be then calculated for each increment of the simulation. Notice that as shown in Fig. 17, the two forces are not co-linear, and furthermore, they continuously rotate relatively to each other during the loading (see the image sequence of Fig. 11). However, the perpendicular force coefficient $\alpha_{\perp}$ determined above includes these effects by construction. This coefficient remains relatively constant during the simulation, with the variation found to be less than $5 \%$ over the portion of the simulation where plane-strain conditions prevail. A plot of the evolution of the coefficient $\alpha_{\perp}$ is given in Fig. 18a.

It is reasonable to assume that $\alpha_{\perp}$ depends more strongly on the geometry and boundary conditions of the problem (e.g., the location of the test-section on the Dshaped mandrel, the geometry of the front and back sides of the specimen, etc.) and the specimen-mandrel friction, rather than the specifics of the material anisotropy. To assess the accuracy of this assumption, the simulations were repeated with the Hill 1948 material model (e.g., Banabic, 2009). This is not proposed as a suitable yield surface for aluminum alloys, but rather as a straightforward way to introduce anisotropy in this numerical exercise. The anisotropic parameters selected $(F=H=0.7813, G=$ 0.2188 , and $L=M=N=1$ ) represented a visibly distorted yield surface, to accentuate the effect of anisotropy (see Dick, 2014). The results are included in Fig. 18a and confirm that there is practically no effect of the material anisotropy on $\alpha_{\perp}$. Hence the results of the FEA model using the von Mises material model can be applied to the experiment of a material with unknown anisotropy. This coefficient is then applied to the 
experimental load cell reading to determine the perpendicular force component passing through the specimen's test-section during the experiment.

The same methodology and material models are used in the $15^{\circ}, 30^{\circ}$, and $45^{\circ}$ RPST FEA models to determine a parallel force coefficient $\alpha_{\| l}$. The parallel force is the component of net force through the test-section that is aligned parallel to the crosssection. A plot of $\alpha_{\|}$is given in Fig. 18b, showing a limited variation throughout the meaningful part of the simulation (i.e., when plane-strain conditions prevail) and practically no effect from the material anisotropy. With these two force corrections determined, the average normal and shear true stresses on the test-section during the RPST experiment can be simply determined as follows:

$$
\begin{gathered}
\sigma_{\perp}=\frac{F_{\perp}}{A}=\alpha_{\perp} \frac{F_{\mathrm{LC}}}{A} \\
\tau=\frac{F_{\|}}{A}=\alpha_{\|} \frac{F_{\mathrm{LC}}}{A}
\end{gathered}
$$

In these equations, the cross-sectional area ' $A$ ' is determined by monitoring the in-plane strains in the test-section through use of the 3D DIC and invoking incompressibility to find the through-thickness plastic strain (Dick, 2014).

\section{b2. Correction for the stress non-uniformity}

For the normal and shear stresses on the test-section, the procedure described above establishes a single value for the entire test-section. However, as shown in Fig. $4 \mathrm{c}$, the stress across the test-section is non-uniform. Several methods were attempted to correct the stress non-uniformity and will be briefly outlined here (for more details, see Dick, 2014). 
The first method attempted was to consider the test-section as being made up of 'strands' of material and then determine the load partitioning between strands with the aid of the full-field strain data from the DIC. A shortcoming of this method is that the DIC fields do not extend across the entire test-section, since image correlation close to the free edges in problematic. Hence there is an unknown amount of error in this approach. Furthermore, correlating the strain fields to stresses requires the adoption of a yield function, which is the unknown rather than the input in this work.

Another attempt at correcting the stress non-uniformity was to approximate the entire test-section to be at an equal level of plastic work (recall that we are working with "work density", rather than "work" itself), determining the plastic work at the edge of the test-section where the material is in uniaxial tension, and then using this plastic work and the known strain fields from the DIC to estimate the stresses in the remainder of the test-section. This approach suffers from both drawbacks mentioned above. In addition, the assumption of equal plastic work density everywhere in the test-section proved to be quite inaccurate.

In the end, the method that was adopted to correct for the stress non-uniformity was the use of an inverse finite element analysis. This method still requires the assumption of a yield function for the material, but is free of the further uncertainties and assumptions of the two previous approaches. Furthermore, it is anticipated that the plane-strain condition in the central region of the test-section is mainly arising due to structural confinement; in other words, it depends more on the geometry and less on the material anisotropy. The error involved in this assumption is also assessed in this section. It should be noted here that the method of (An et al., 2004), whereby the 
responses of specimens with different widths of the test-section are compared to each other and allow an assessment the effect of the two edges was not pursued here. It appears that this method could be used in thinner tubes as an alternative to what is described here.

Using the FEA model and comparing the actual true stress variation across the testsection (i.e., from element integration points) to the average stress as it would be determined experimentally (Eqns. 3a and 3b), establishes the correction factor for the stress non-uniformity. Figure 18 shows the perpendicular true stress correction identified as the double arrows for the $0^{\circ}$ and $45^{\circ}$ RPST specimens. The correction factor is determined at the center of the test-section as this is the location of plane-strain tension, as well as the location where the work-conjugate DIC data will be reported from. It was determined that the correction factor changes slightly with deformation, hence it must be determined at various levels of plastic deformation using the FEA model (Fig. 19 is for a plastic work level of $5 \mathrm{MPa}$ ). Furthermore, as shown in Fig. 19, each test specimen requires a different set of correction factors.

The normal true stress perpendicular to the test-section is determined in this way. The normal true stress parallel to the test-section, which is induced to materialize the plane-strain constraint, is then assumed to be half of the perpendicular normal stress, per the von Mises prediction. This is a known source of error in this analysis, as the material is anisotropic and hence the two stresses are not necessarily at a 2:1 ratio.

The same procedure was used to determine the correction for the non-uniform true shear stresses, shown in Fig. 20. Interestingly, as the rotation of the test-section increases, the shear stress distribution along the test-section becomes more uniform, so 
that the corresponding correction factor is reduced. The final set of correction factors is listed in Table 1.

The correction for the stress non-uniformity as described is based on a finite element model using a von Mises material model. To assess the accuracy of this assumption, the simulations were repeated with the same Hill 1948 material model as in Section b1. Recall that the yield surface that this model predicts, as calibrated, is visibly distorted. The difference between the von Mises and Hill results is shown in Fig. 19 and the new set of corrections is included in Table 1. In most cases, the numerical values are similar for the two material models, deteriorating as the test-section angle with the tube generatrix increases. It can be concluded that for the real material, which is expected to be somewhere in-between the two, correction factors that are bounded by the values of Table 1 should be used. However, since we prefer to be systematic and avoid bias towards any material model, we opted to use the correction factors from the von Mises material model in the remainder of this work. In principle, once a suitable yield function has been calibrated for Al-6061-T4, these assumptions could be revisited with the benefit of this yield function and refined in a $2^{\text {nd }}$ round of RPST data post-processing. Furthermore, it is interesting to note that this procedure for correcting the average true stress for the non-uniformity of the stress field can be applied not only to RPST but also to any similar specimen geometry.

The force coefficients and stress correction factors are applied to the experimental data to evaluate the true stress-state in the center of the specimen's test-section. The stress-state on each specimen's parallel-perpendicular coordinate system is rotated to 
the tube's axial-hoop coordinate system using the standard 2D stress transformation equations. This process is depicted in Fig. 21.

\section{ANISOTROPY OF AI-6061-T4 TUBES}

\section{a. Overview}

The true stress-state of each RPST specimen is determined as described in the previous section. The result can be then plotted in the axial-hoop plane-stress space of the tube's coordinate system $\left(\sigma_{\mathrm{a}}-\sigma_{\mathrm{h}}-\tau_{\mathrm{ah}}\right)$ as shown in Fig. 21 . Since the $15^{\circ}, 30^{\circ}$ and $45^{\circ}$ RPST specimens result in stress-states that have shear, they lie in 3D in the $\left(\sigma_{\mathrm{a}^{-}}\right.$ $\left.\sigma_{\mathrm{h}}-\tau_{\mathrm{ah}}\right)$ space. The two uniaxial tests performed previously, one in the axial direction of the tube (performed on a strip) and the other in the hoop direction (performed using the Ring Hoop Tension Test, Dick and Korkolis, 2014a) are also plotted in this stress space. The post-processing procedure described in Section 4 above ensures that the plastic work level for each specimen is properly and consistently determined, so that at any given level of plastic work, the results from the 4 RPST specimens lie on the same plastic work contour and can be directly compared to each other. Recall that under the assumption that the center of each subsequent yield surface does not move, the plastic work contours are identical to the subsequent yield surfaces of the deforming material (Hill \& Hutchinson, 1992, Hill et al., 1994).

\section{b. Material models and fitting procedure}

As is well-accepted in the literature, quadratic yield functions such as the von Mises and Hill 1948 are not suitable models for aluminum alloys. Instead, non-quadratic yield 
functions offer a much closer match to experimental results (e.g., Dunand et al., 2012, Giagmouris et al., 2010, Korkolis and Kyriakides, 2008, 2011b, Kuwabara et al., 2005, among many others). Two established anisotropic material models were fitted to the RPST data, which are presented in Table 2 at 7 levels of constant plastic work. The first model is the 8-parameter anisotropic Yld2000-2D yield function (Barlat et al., 2003), which represents plane-stress states. This yield function is based on the non-quadratic, isotropic Hosford yield function (Hosford, 1972) written in terms of the principal values of the stress deviator. As is customary for FCC alloys, the exponent of this yield function was set to 8 (Barlat et al., 2003, Korkolis and Kyriakides, 2008, 2011). Anisotropy is introduced by two linear, convexity-preserving transformations on the stress tensor. The two transformations involve 8 anisotropy parameters, which can be adjusted to match the experimental data. As has been shown before (e.g., Korkolis and Kyriakides, 2008, $2009,2011 b)$ this yield function is quite flexible and can represent the anisotropy of aluminum alloys quite accurately. In the present work, the anisotropy parameters were determined using an optimization algorithm. An objective function that, following the recommendations of (Barlat et al., 2003) represented the sum of the squared distances of the experiments from their closest-point projections on the yield surface was constructed. This was minimized using unconstrained nonlinear optimization. This process was repeated for several levels of plastic work. The resulting anisotropy parameters are given in Table 3. A histogram of the distances of the closest-point projections of the Yld2000-2D model to the experimental data is given in Fig. 22. Also included are the results for the von Mises model. The distances were normalized with the flow stress from the uniaxial test (performed on a strip) at the same level of plastic 
work. The histogram shows that the Yld2000-2D model offers a systematically better fit to the experimental data over the von Mises model.

The second material model that was fitted to the data was the 18-parameter anisotropic Yld2004-3D yield function, which can be used with fully-3D stress-states (Barlat et al., 2005, Yoon et al., 2006). This function is also based on the non-quadratic, isotropic Hosford model. The anisotropy is again introduced through two linear, convexity-preserving transformations. However, in contrast to the Yld2000-2D case, the $3^{\text {rd }}$ principal deviatoric stress is preserved in this process. Each linear transformation now involves 9 anisotropy parameters, for a total of 18 independent parameters. In the present work, these were optimized in the same way as for the Yld2000-2D case, and at the same level of plastic work. The resulting parameters are given in Table 4 and the quality of the fit to the experimental data over the von Mises model is included in the histogram of Fig. 22. The Yld2004-3D model offers a better fit to the experimental data than the von Mises model. The quality of the fit is comparable to the Yld2000-2D model, except for the uniaxial strip experiment. This lack of agreement to the uniaxial data (strip) is because all experimental points are treated equally by this algorithm, and hence a better fit was obtained with the existing parameters, rather than by forcing the model to pass from the uniaxial point.

In conclusion, both of the anisotropic yield functions fit the experimental data much better than the von Mises model, which especially underestimates the material behavior under stress-states close to pure hoop tension. This is in agreement with the responses shown in Fig. 1 b. 


\section{Representation of the anisotropy of Al-6061-T4}

Since the $15^{\circ}, 30^{\circ}$ and $45^{\circ}$ RPST experimental data have out-of-plane shear, three different approaches for visualizing the resulting plastic work contours and the quality of fit to the experiments were pursued. The first way is the full representation of the yield functions in the true $\left(\sigma_{\mathrm{a}}-\sigma_{\mathrm{h}}-\tau_{\mathrm{ah}}\right)$ space. This is shown in Figs 23a and b. Included in this figure are the experiments with open circles and their projections on the yield surfaces with filled circles. It can be seen that these two sets of data almost overlap, indicating that the fits are very accurate. However, while accurate as plots, these 3D views do not allow for an easy quantitative assessment of the quality of the fit.

The second way to represent the anisotropic yield functions is a 2-D plot $\left(\sigma_{\mathrm{a}}-\sigma_{\mathrm{h}}\right)$ of lines of constant shear stress, which will be termed isoshear lines, i.e., lines of $\tau_{\mathrm{ah}}=$ const. Figure 24a shows the Yld2000-2D yield surface fitted to the 6 experimental data points recorded from RPST and RHTT experiments, at a plastic work level of 5 $\mathrm{MPa}$. Each ring-like contour represents an isoshear at the same level as the shear stress in the experimental data. The experimental data and the isoshear lines have been color coded to identify each pair. The shear stresses for the RPST $15^{\circ}$ (red), $30^{\circ}$ (blue) and $45^{\circ}$ (green) experimental data points and isoshears are at 18, 67 and 69 $\mathrm{MPa}$, respectively. The outermost isoshear line (brown) corresponds to zero shear stress and therefore is the in-plane $\left(\sigma_{\mathrm{a}}-\sigma_{\mathrm{h}}\right)$ yield surface. As shown in Fig. 24a, each isoshear line captures its corresponding experimental data point very accurately. Of course, due to the lack of enough in-plane stress paths, the overall shape of the yield surface in the $\left(\sigma_{\mathrm{a}}-\sigma_{\mathrm{h}}\right)$ plane must be assumed. Ideally, the proposed RPST experiments 
would be used in collaboration with another testing method, such as loading of the tubes under axial load and internal pressure (Korkolis and Kyriakides, 2008, 2009, Khan et al., 2010b, Korkolis et al., 2010, Kuwabara, 2007, 2014), to supplement the in-plane data with out-of-plane data that include shear. Alternatively, additional data points could be generated by crystal plasticity calculations for the given texture of the Al-6061-T4 tubes.

The fact that the lack of in-plane points generates an underdetermined solution for the yield function to represent the material anisotropy is illustrated in Fig. 24b, which shows the calibration of the Yld2004-3D model to the same experimental data (also at plastic work level of $5 \mathrm{MPa}$ ) as in Fig. 24a. As in the case of the Yld2000-2D, this model can very accurately capture the experimental data (see Fig. 22). However, due to the high flexibility and greater number of fitting coefficients, the overall shape of the yield surface for the 3-D model is seen to differ from the results of the 2-D model in Fig. 24a. Of course, it is possible to generate "dummy" data points for any stress combination using one yield function and then use these to supplement the experimental points and calibrate the other yield function so that their in-plane shapes match very closely. This point was not pursued here (for the results of this procedure, see Dick, 2014), precisely to highlight the multiple solutions (local minima in the optimization procedure) that these very advanced and flexible yield functions admit.

The nature of these plots makes it very difficult to visualize the evolution of the plastic work contours. Therefore, a third way of representing these results was pursued, by displaying them on the Haigh-Westergaard, or $\pi$-plane. Since each yield function calibration is valid only in the frame that it is calibrated at, the experimental data were 
transformed from the $\left(\sigma_{\mathrm{a}}-\sigma_{\mathrm{h}}-\tau_{\mathrm{ah}}\right)$ space to the principal space, fitted there using the same method as described above and then projected on the $\pi$-plane. Only the Yld2000$2 \mathrm{D}$ model was used for this process, as the lack of enough experimental data points makes it impossible to determine the shape of some parts of the yield surface (see Figs. $24 \mathrm{a}$ and $\mathrm{b})$. Hence we opted to avoid showing contradicting yield surfaces and their evolution in the regions where no data for calibration exist. The results are shown in Fig. 25. Also included in Fig. 25 are plastic work contours at multiple levels of plastic deformation, which show the differential-work-hardening (Hill \& Hutchinson, 1992, Hill et al., 1994) that this alloy exhibits (for similar results on a different 6000-series aluminum alloy see Khan et al., 2009, 2010a, Korkolis and Kyriakides 2008, 2009). Clearly, this approach offers easier visualization of the evolution of plastic anisotropy and of the performance of a material model in matching the experiments. Furthermore, it can be used to direct the design of additional RPST specimens. For example, it appears that notch orientations between the existing $0^{\circ}$ and $15^{\circ}$ would provide significant information on the shape of the work contours. Finally, for comparison purposes, the von Mises model, which on the $\pi$-plane is represented as a circle, is included in Fig. 25 at the two extreme values of the plastic work (0.1 and $10 \mathrm{MPa}$, respectively).

\section{CONCLUSIONS}

A new method for the testing of thin-walled tubes under combined tension and shear using a universal testing machine was proposed. The specimens resemble plane-strain tension specimens for sheets (e.g., Mohr and Oswald, 2008, Tardif and Kyriakides, 2012, Lou et al., 2014), but wrapped over two rigid, D-shaped mandrels. As the 
mandrels are parted in a universal testing machine, the test-section of the specimens deforms under plane-strain. A family of RPST specimens was designed, by rotating the orientation of the test-section with respect to the tube generatrix, thus enabling to generate infinite combinations of tension and shear.

The paper began by describing the design of the specimen family with the aid of numerical simulation. The numerical model was also used to compare the performance of the RPST specimens to existing specimen designs for plane-strain tension of sheets. This exercise verified that the RPST specimens behave similarly to the existing designs for sheets. Subsequently, RPST experiments on extruded Al-6061-T4 tubes were performed. Four orientations of the test-section were investigated: $0^{\circ}, 15^{\circ}, 30^{\circ}, 45^{\circ}$. With the aid of DIC, the strain fields inside the test-section were probed, which confirmed that plane-strain conditions exist for a significant part of the experiment.

The proposed RPST specimens were then used to establish the anisotropy of the Al6061-T4 tubes. The true stresses that develop in the test-section were determined from the load cell reading after undergoing two corrections: one for the orientation of the testsection to the vertical and a second for the non-uniformity of the stress distribution. While the former is specific to the RPST geometry, the latter is applicable to any planestrain tension specimen. It was shown that anisotropy had no effect on the former procedure, but did have an effect on the latter, albeit limited. Furthermore, the normal stress parallel to the test-section was determined using the von Mises assumption. Combining these stresses to the strains acquired by the DIC, the plastic work could be calculated and used to generate contours of constant plastic work. 
The plastic work contours were then fitted with two non-quadratic, anisotropic yield functions, the Yld2000-2D and the Yld2004-3D. The quality of these fits was compared to the von Mises one and found to be much closer to the experimental data. Furthermore, the evolution of the plastic work contours was investigated with the Yld2000-2D model using the $\pi$-plane representation. The parameters of this model were adjusted with plastic work so that accurate representation of the evolving work contours was achieved.

The family of RPST specimens introduced in this paper is proposed as a supplement, rather than a replacement of existing methods for probing the anisotropy of thin-walled tubes. For example, with minimal requirements for additional equipment, they can supplement the data from tube inflation under axial load and internal pressure, which generates biaxial principal stress-states, with out-of-plane data under combined tension and shear.

\section{ACKNOWLEDGEMENTS}

This work was performed under the GOALI grant CMMI 1031169 from the National Science Foundation, whose support is acknowledged with thanks. The tubes were provided by Dr. Cedric Xia of Ford Motor Company. We thank Alex Roy for help with machining of the specimens. Special thanks to Dr. Holger Aretz of Hydro Aluminium Rolled Products GmbH for helpful discussions on the representation of the anisotropy. 


\section{APPENDIX A}

In this appendix, an engineering drawing of the proposed RPST specimen $\left(0^{\circ}\right)$ is presented. The $15^{\circ}, 30^{\circ}$ and $45^{\circ}$ specimens maintain the same overall and test-section dimensions, with the later rotated accordingly. 


\section{REFERENCES}

An, Y.G., Vegter, H. and Elliott, L. (2004). A novel and simple method for the measurement of plane strain work hardening. J. Matl's Proc. Tech., 155, 16161622.

Aretz, H. and Barlat, F. (2013). New convex yield functions for orthotropic metal plasticity. Int'l J. Non-Linear Mech., 51, 97-111.

Banabic, D. (2009). Sheet metal forming processes. Springer-Verlag Berlin Heidelberg.

Barlat, F., Brem, J.C., Yoon, J.W., Chung, K., Dick, R.E., Lege, D.J., Pourboghrat, F., Choi, S.-H. and Chu, E. (2003). Plane Stress Yield Function for Aluminum Alloy Sheets-Part I: Theory, Int'l J. Plasticity 19, 1297-1319.

Barlat, F., Aretz, H., Yoon, J.W., Karabin, M.E., Brem, J.C., Dick, R.E. (2005). Linear transformation-based anisotropic yield functions. Int'l J. Plasticity 21, 1009-1039.

Dick, C.P. (2014). The Ring Hoop Tension and the Ring Plane Strain Tension tests for measuring the anisotropy of Al-6061-T4 tubes. MS Thesis, Univ. New Hampshire.

Dick, C.P. and Korkolis, Y.P. (2014a). Mechanics and Full-Field Deformation Study of the Ring Hoop Tension Test. Int'l J. Solids \& Struct. 51, 3042-3057. 
Dick, C.P. and Korkolis, Y.P. (2014b). Strength and ductility evaluation of cold-welded seams in aluminum tubes extruded through porthole dies. Mat'ls \& Design, 67, 631-636.

Dunand, M., Maertens, A. P., Luo, M. and Mohr, D. (2012). Experiments and modeling of anisotropic aluminum extrusions under multi-axial loading-Part I: Plasticity. Int'l J. Plasticity, 36, 34-49.

Giagmouris, T., Kyriakides, S., Korkolis, Y.P. and Lee, L.-H. (2010). On the localization and failure in aluminum shells due to crushing-induced bending and tension. Int'l J. Solids \& Struct. 47, 2680-2692.

Hill, R. and Hutchinson, J.W. (1992). Differential hardening in sheet metal under biaxial loading: A theoretical framework. ASME J. Applied Mechanics 59, 1-9.

Hill, R., Hecker, S.S. and Stout, M.G. (1994). An investigation of plastic flow and differential work hardening in orthotropic brass tubes under fluid pressure and axial load. Int'l J. Solids \& Structures 21, 2999-3021.

Hosford, W.F. (1972). A Generalized Isotropic Yield Criterion, ASME J. Appl. Mech. 309, pp. 607-609. 
Khan, A. S., Kazmi, R., Pandey, A. and Stoughton, T. (2009). Evolution of subsequent yield surfaces and elastic constants with finite plastic deformation. Part-I: A very low work hardening aluminum alloy (Al6061-T6511). Int'l J. Plasticity, 25(9), 16111625.

Khan, A. S., Pandey, A. and Stoughton, T. (2010a). Evolution of subsequent yield surfaces and elastic constants with finite plastic deformation. Part II: A very high work hardening aluminum alloy (annealed $1100 \mathrm{Al}$ ). Int'l J. Plasticity, 26(10), 14211431.

Khan, A. S., Pandey, A. and Stoughton, T. (2010b). Evolution of subsequent yield surfaces and elastic constants with finite plastic deformation. Part III: Yield surface in tension-tension stress space (Al 6061-T 6511 and annealed $1100 \mathrm{Al}$ ). Int'l J. Plasticity, 26(10), 1432-1441.

Korkolis, Y.P. and Kyriakides, S. (2008). Inflation and burst of anisotropic aluminum tubes for hydroforming applications. Int'l J. Plasticity 24/3, 509-543.

Korkolis, Y.P. and Kyriakides, S. (2009). Path-dependent failure of inflated aluminum tubes. Int'l J. Plasticity 25, 2059-2080. 
Korkolis, Y.P., Kyriakides, S., Giagmouris, T., Lee, L.-H. (2010). Constitutive modeling and rupture predictions of Al-6061-T6 tubes under biaxial loading paths. ASME J. Appl. Mech. 77, 64501-1 to -5 .

Korkolis, Y. P. and Kyriakides, S. (2011a). Hydroforming of anisotropic aluminum tubes. Part I: Experiments. Int. J. Mech. Sci. 53, 75-82.

Korkolis, Y.P. and Kyriakides, S. (2011b). Hydroforming of anisotropic aluminum tubes. Part II: analysis. Int'l J. Mech. Sci. 53, 83-90.

Kuwabara, T., Yoshida, K., Narihara, K. and Takahashi, S. (2005). Anisotropic plastic deformation of extruded aluminum alloy tube under axial forces and internal pressure. Int. J. Plasticity 21, 101-117.

Kuwabara, T. (2007). Advances in experiments on metal sheets and tubes in support of constitutive modeling and forming simulations. Int. J. Plasticity 23, 385-419.

Kuwabara, T., Hashimoto, K., lizuka, E. and Yoon, J. W. (2011). Effect of anisotropic yield functions on the accuracy of hole expansion simulations. J. Mat'ls Proc. Tech., 211(3), 475-481. 
Kuwabara, T. (2014). Biaxial Stress Testing Methods for Sheet Metals. In Comprehensive Materials Processing; Van Tyne, C. J., Ed.; Elsevier Ltd.; Vol. 1, pp 95-111.

Link, T.M., Koss, D.A. and Motta, A.T. (1998). Failure of Zircalloy cladding under transverse plane-strain deformation. Nucl. Eng. \& Des. 186, 379-394.

Lou, Y., Yoon, J. W. and Huh, H. (2014). Modeling of shear ductile fracture considering a changeable cut-off value for stress triaxiality. Int. J. Plasticity, 54, 56-80.

Luo, M., Dunand, M. and Mohr, D. (2012). Experiments and modeling of anisotropic aluminum extrusions under multi-axial loading-Part II: Ductile fracture. Int. J. Plasticity, 32, 36-58.

Mohr, D. and Oswald, M. (2008). A new experimental technique for the multi-axial testing of advanced high strength steel sheets. Exp. Mech. 48, 65-77.

Mohr, D., Dunand, M. and Kim, K. H. (2010). Evaluation of associated and nonassociated quadratic plasticity models for advanced high strength steel sheets under multi-axial loading. Int. J. Plasticity, 26(7), 939-956. 
Soare, S., Yoon, J. W. and Cazacu, O. (2008). On the use of homogeneous polynomials to develop anisotropic yield functions with applications to sheet forming. Int. J. Plasticity, 24(6), 915-944.

Stoughton, T. B. and Yoon, J. W. (2011). A new approach for failure criterion for sheet metals. Int. J. Plasticity, 27(3), 440-459.

Tardif, N. and Kyriakides, S. (2012). Determination of anisotropy and material hardening for aluminum sheet metal. Int'l J. Solids \& Struct. 49(25), 3496-3506.

Yoon, J. W., Barlat, F., Dick, R. E. and Karabin, M. E. (2006). Prediction of six or eight ears in a drawn cup based on a new anisotropic yield function. Int. J. Plasticity, 22(1), 174-193.

Yoshida, F., Hamasaki, H. and Uemori, T. (2013). A user-friendly 3D yield function to describe anisotropy of steel sheets. Int. J. Plasticity, 45, 119-139. 
TABLES

Table 1: Correction factors for the normal and shear stress perpendicular to the testsection.

\begin{tabular}{|c|c|c|c|c|c|c|c|c|}
\hline \multirow{2}{*}{$W^{p}=5 \mathrm{MPa}$} & \multicolumn{2}{|c|}{ RPST $0^{\circ}$} & \multicolumn{2}{c|}{ RPST $15^{\circ}$} & \multicolumn{2}{c|}{ RPST $30^{\circ}$} & \multicolumn{2}{c|}{ RPST $45^{\circ}$} \\
\cline { 2 - 9 } & $\mathrm{vM}$ & Hill & $\mathrm{vM}$ & Hill & $\mathrm{vM}$ & Hill & $\mathrm{vM}$ & Hill \\
\hline Normal & 0.89 & 0.90 & 0.83 & 0.81 & 0.84 & 0.76 & 0.81 & 0.62 \\
\hline Shear & - & - & 1.27 & 1.39 & 1.11 & 1.26 & 1.08 & 1.19 \\
\hline
\end{tabular}

Table 2: Stresses on experimental work contours of Al-6061-T4.

\begin{tabular}{|c|c|c|c|c|}
\hline & $W^{p}(\mathrm{MPa})$ & $\sigma_{\mathrm{a}}(\mathrm{MPa})$ & $\sigma_{\mathrm{h}}(\mathrm{MPa})$ & $\tau_{\mathrm{ah}}(\mathrm{MPa})$ \\
\hline \hline & 0.1 & 139.28 & 0.00 & 0.00 \\
\cline { 2 - 5 } & 0.5 & 154.90 & 0.00 & 0.00 \\
\cline { 2 - 5 } & 1 & 162.74 & 0.00 & 0.00 \\
\cline { 2 - 5 } $\begin{array}{c}\text { Uniaxial (Dick \& } \\
\text { Korkolis, 2014) }\end{array}$ & 3 & 185.24 & 0.00 & 0.00 \\
\cline { 2 - 5 } & 5 & 202.65 & 0.00 & 0.00 \\
\cline { 2 - 5 } & 7 & 217.63 & 0.00 & 0.00 \\
\cline { 2 - 5 } & 10 & 235.06 & 0.00 & 0.00 \\
\hline \multirow{3}{*}{$\begin{array}{c}\text { Ring Hoop Tension } \\
\text { Test (Dick \& } \\
\text { Korkolis, 2014) }\end{array}$} & 0.1 & 0.00 & 109.57 & 0.00 \\
\cline { 2 - 5 } & 1 & 0.00 & 129.51 & 0.00 \\
\cline { 2 - 5 } & 3 & 0.00 & 136.46 & 0.00 \\
\hline
\end{tabular}




\begin{tabular}{|c|c|c|c|c|}
\hline & 5 & 0.00 & 173.70 & 0.00 \\
\hline & 7 & 0.00 & 187.03 & 0.00 \\
\hline & 10 & 0.00 & 203.11 & 0.00 \\
\hline \multirow{7}{*}{ RPST $-0^{\circ}$} & 0.1 & 136.32 & 0.00 & 68.16 \\
\hline & 0.5 & 164.52 & 0.00 & 82.26 \\
\hline & 1 & 177.73 & 0.00 & 88.87 \\
\hline & 3 & 204.35 & 0.00 & 102.18 \\
\hline & 5 & 220.22 & 0.00 & 110.11 \\
\hline & 7 & 231.85 & 0.00 & 115.93 \\
\hline & 10 & 244.59 & 0.00 & 122.29 \\
\hline \multirow{7}{*}{ RPST $-15^{\circ}$} & 0.1 & 75.20 & 83.94 & -45.48 \\
\hline & 0.5 & 95.69 & 106.81 & -57.87 \\
\hline & 1 & 104.32 & 116.44 & -63.09 \\
\hline & 3 & 122.59 & 136.84 & -74.14 \\
\hline & 5 & 134.17 & 149.76 & -81.14 \\
\hline & 7 & 142.99 & 159.61 & -86.48 \\
\hline & 10 & 151.44 & 169.04 & -91.59 \\
\hline \multirow{7}{*}{ RPST $-30^{\circ}$} & 0.1 & 101.42 & 19.86 & -46.88 \\
\hline & 0.5 & 114.08 & 22.34 & -52.74 \\
\hline & 1 & 122.20 & 23.92 & -56.49 \\
\hline & 3 & 144.77 & 28.34 & -66.93 \\
\hline & 5 & 160.33 & 31.39 & -74.12 \\
\hline & 7 & 171.44 & 33.56 & -79.25 \\
\hline & 10 & 183.42 & 35.91 & -84.79 \\
\hline
\end{tabular}




\begin{tabular}{|c||c||c|c|c|}
\hline \hline & 0.1 & 93.35 & -20.29 & -12.18 \\
\cline { 2 - 5 } & 0.5 & 108.58 & -23.60 & -14.16 \\
\cline { 2 - 5 } & 1 & 117.17 & -25.47 & -15.28 \\
\cline { 2 - 5 } RPST - 45 & 3 & 138.89 & -30.19 & -18.12 \\
\cline { 2 - 5 } & 5 & 153.90 & -33.46 & -20.07 \\
\cline { 2 - 5 } & 7 & 166.45 & -36.18 & -21.71 \\
\cline { 2 - 5 } & 10 & 182.34 & -39.64 & -23.78 \\
\hline \hline
\end{tabular}

Table 3: Anisotropy parameters of Yld2000-2D ( $k=8)$ for Al-6061-T4.

\begin{tabular}{|c||c|c|c|c|c|c|c|}
\hline $\begin{array}{c}W^{p} \\
(\mathrm{MPa})\end{array}$ & 0.1 & 0.5 & 1 & 3 & 5 & 7 & 10 \\
\hline \hline$\alpha_{1}$ & 0.724 & 0.848 & 0.839 & 0.859 & 0.880 & 0.888 & 0.914 \\
\hline$\alpha_{2}$ & 1.687 & 1.501 & 1.521 & 1.478 & 1.440 & 1.423 & 1.380 \\
\hline$\alpha_{3}$ & 1.233 & 1.061 & 1.050 & 1.063 & 1.076 & 1.081 & 1.057 \\
\hline$\alpha_{4}$ & 1.098 & 1.016 & 0.982 & 0.975 & 0.994 & 1.016 & 1.048 \\
\hline$\alpha_{5}$ & 0.943 & 0.903 & 0.890 & 0.890 & 0.887 & 0.887 & 0.886 \\
\hline$\alpha_{6}$ & 0.969 & 1.023 & 0.948 & 0.938 & 0.947 & 0.941 & 0.949 \\
\hline$\alpha_{7}$ & 1.257 & 1.317 & 1.293 & 1.196 & 1.164 & 1.172 & 1.184 \\
\hline$\alpha_{8}$ & 1.414 & 0.899 & 0.780 & 0.840 & 0.868 & 0.874 & 0.922 \\
\hline
\end{tabular}


Table 4: Anisotropy parameters of Yld2004-3D $(\mathrm{k}=8)$ for Al-6061-T4.

\begin{tabular}{|c||c|c|c|c|c|c|c|}
\hline $\begin{array}{c}W^{p} \\
(\mathrm{MPa})\end{array}$ & 0.1 & 0.5 & 1 & 3 & 5 & 7 & 10 \\
\hline \hline$c_{12}^{\prime}$ & 1.413 & 1.170 & 1.248 & 1.339 & 1.301 & 1.256 & 1.290 \\
\hline$c_{13}^{\prime}$ & 0.744 & 0.797 & 0.757 & 0.786 & 0.827 & 0.868 & 0.826 \\
\hline$c_{21}^{\prime}$ & 1.110 & 1.235 & 1.154 & 1.141 & 1.168 & 1.198 & 1.154 \\
\hline$c_{23}^{\prime}$ & 1.065 & 0.989 & 0.976 & 0.960 & 0.987 & 1.014 & 1.015 \\
\hline$c_{31}^{\prime}$ & 0.975 & 0.754 & 0.743 & 0.942 & 0.921 & 0.891 & 0.965 \\
\hline$c_{32}^{\prime}$ & 1.157 & 1.010 & 0.990 & 0.919 & 0.952 & 0.981 & 0.996 \\
\hline$c_{44}^{\prime}$ & 1.000 & 1.000 & 1.000 & 1.000 & 1.000 & 1.000 & 1.000 \\
\hline$c_{55}^{\prime}$ & 1.000 & 1.000 & 1.000 & 1.000 & 1.000 & 1.000 & 1.000 \\
\hline$c_{66}^{\prime}$ & 1.164 & 1.320 & 1.242 & 1.071 & 1.124 & 1.125 & 1.085 \\
\hline$c_{12}^{\prime \prime}$ & 0.668 & 1.063 & 0.967 & 0.931 & 0.959 & 0.997 & 0.935 \\
\hline$c_{13}^{\prime \prime}$ & 1.074 & 0.705 & 0.701 & 0.926 & 0.898 & 0.876 & 0.960 \\
\hline$c_{21}^{\prime \prime}$ & 1.160 & 1.148 & 1.185 & 1.104 & 1.123 & 1.141 & 1.073 \\
\hline$c_{23}^{\prime \prime}$ & 1.072 & 1.014 & 0.961 & 1.045 & 1.017 & 1.012 & 1.100 \\
\hline$c_{31}^{\prime \prime}$ & 0.728 & 0.671 & 0.663 & 0.870 & 0.853 & 0.842 & 0.895 \\
\hline$c_{32}^{\prime \prime}$ & 1.102 & 1.073 & 1.034 & 0.992 & 1.031 & 1.063 & 1.053 \\
\hline$c_{44}^{\prime \prime}$ & 1.000 & 1.000 & 1.000 & 1.000 & 1.000 & 1.000 & 1.000 \\
\hline$c_{55}^{\prime \prime}$ & 1.000 & 1.000 & 1.000 & 1.000 & 1.000 & 1.000 & 1.000 \\
\hline$c_{66}^{\prime \prime}$ & 1.432 & 1.278 & 1.281 & 1.073 & 1.082 & 1.080 & 1.092 \\
\hline \hline & & & & & & & \\
\hline
\end{tabular}




\section{FIGURE CAPTIONS}

Figure 1 - a) Ring Hoop Tension Test specimen and D-shaped mandrel assembly. b) Nominal stress-strain responses in the axial and hoop directions for the Al-6061-T4 material.

Figure 2 - Ring Plane Strain Tension specimen design.

Figure 3 - Plane-strain tension specimen designed for sheets (adapted from Tardif and Kyriakides, 2012). Dimensions are in inches $(1 \mathrm{inch}=25.4 \mathrm{~mm})$.

Figure $4-$ a) Finite element model of the Ring Plane Strain Tension (RPST) $0^{\circ}$ specimen. b) Comparison of the true strain distributions of the RPST and sheet plane-strain specimen designs. c) Comparison of the true stress distributions of the RPST and sheet plane-strain specimen designs.

Figure 5 - Additional RPST specimen designs: a) $15^{\circ}$ b) $30^{\circ}$ c) $45^{\circ}$

Figure 6 - Validation of plane-strain conditions for all RPST specimens.

Figure 7 - Effect of friction on the plane-strain condition for the $30^{\circ}$ RPST specimen. 
Figure 8 - Ring Plane Strain Tension $45^{\circ}$ specimen with D-shaped mandrel and grip assembly.

Figure 9 - Load-displacement responses from the RPST experimental testing.

Figure 10 - Detailed investigation of the $30^{\circ}$ RPST load-displacement response.

Figure 11 - a-f) Subsequent images of the $30^{\circ}$ RPST specimen deforming under load, including the failure initiation and the specimen rupture.

Figure 12 - Full parallel nominal strain contours of the RPST specimens from DIC images: a) $0^{\circ}$ b) $15^{\circ}$ c) $30^{\circ}$ d) $45^{\circ}$

Figure 13 - Evolution of the perpendicular and parallel extensional true strains for the $0^{\circ}$ RPST specimen to experimentally validate the plane-strain condition.

Figure $14-a)$ Evolution of the perpendicular and parallel extensional true strains for the $45^{\circ}$ RPST specimen to experimentally validate the plane-strain condition. b) Shear strain distribution and evolution for the $45^{\circ}$ RPST specimen.

Figure 15 - Fractured RPST $0^{\circ}$ specimen with the DIC paint removed to show the deformation-induced roughening. 
Figure 16 - Flowchart of the post-processing procedure for the RPST experiments.

Figure 17 - Relative orientation of the load cell force and the force perpendicular to the test-section: a) isometric view. b) r- $\theta$ plane. c) r-z plane.

Figure 18 - Evolution of: a) the perpendicular and b) the parallel force corrections during loading.

Figure 19 - Non-uniformity correction of the normal true stress that is perpendicular to the test-section for: a) $0^{\circ}$ b) $45^{\circ}$ specimens.

Figure 20 - Non-uniformity correction for true shear stress for: a) $15^{\circ}$ b) $30^{\circ}$ c) $45^{\circ}$ specimens.

Figure 21 - Coordinate system rotation from the parallel-perpendicular system of the test-section to the tube's axial-hoop system.

Figure 22 - Histogram of the distances (closest-point projections) of the fitted yield functions to the experimental data points.

Figure 23 - Three-dimensional views of: a) the Yld2000-2D and b) the Yld2004-3D yield functions fitted to the experimental RPST data. Note that the negative of the shear that the experiments actually have is shown in these plots. 
Figure 24 - Projections of the three-dimensional views of Fig. 23 to the axial-hoop coordinate system, showing the lines of constant shear stress. a) Yld2000-2D and b) Yld2004-3D yield functions.

Figure 25 - Projection of the Yld2000-2D yield function on the $\pi$-plane for increasing levels of plastic deformation. Also shown are the von Mises projections at the two extreme levels of plastic work. Each axis in this plane is the projection of principal stress on the $\pi$-plane, i.e., $\sigma_{i}{ }^{\prime}=\sqrt{2 / 3} \sigma_{i}, i=1,3$.

Figure A1 - Engineering drawing of the $0^{\circ}$ RPST specimen. 


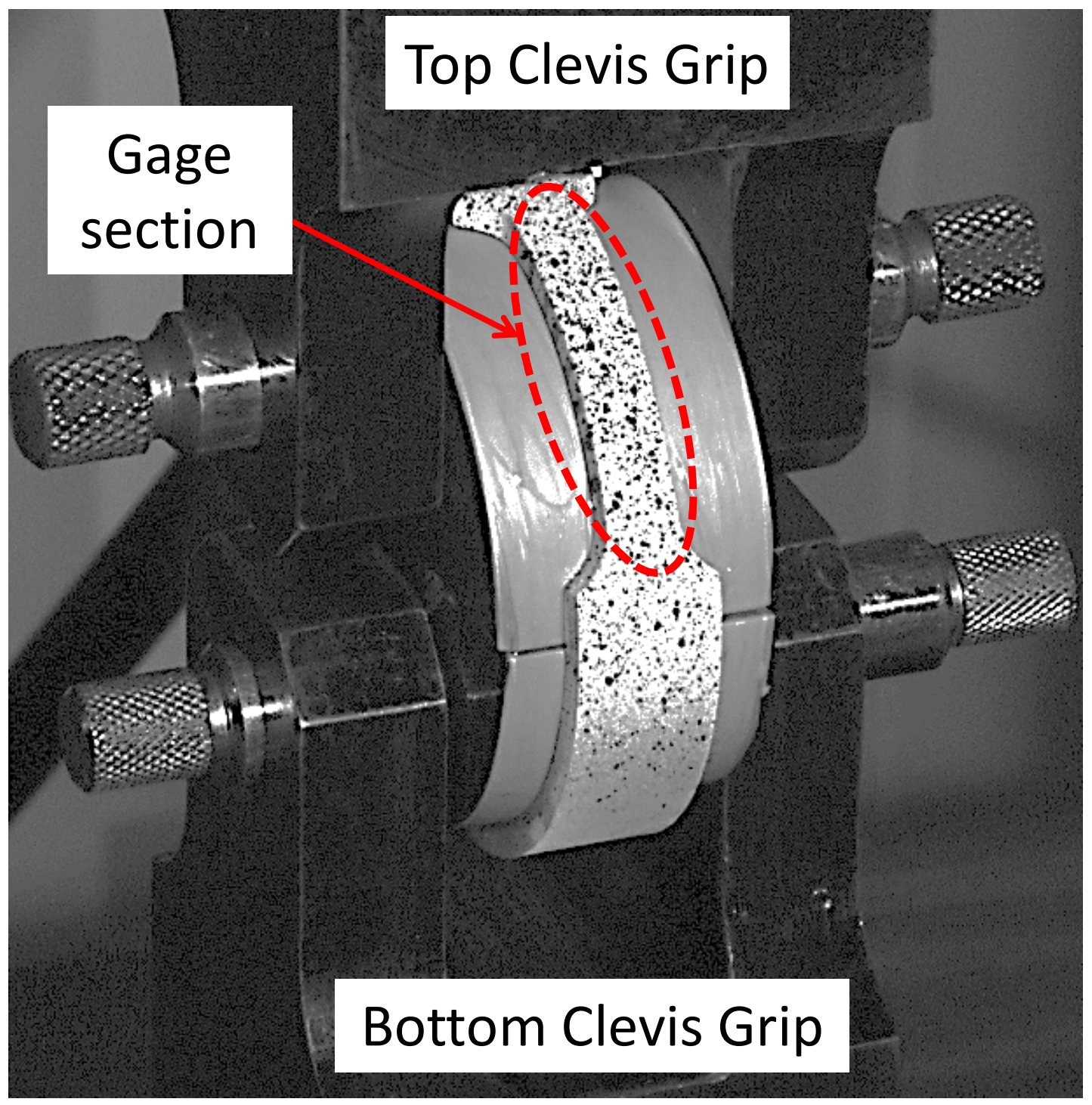

F1a 


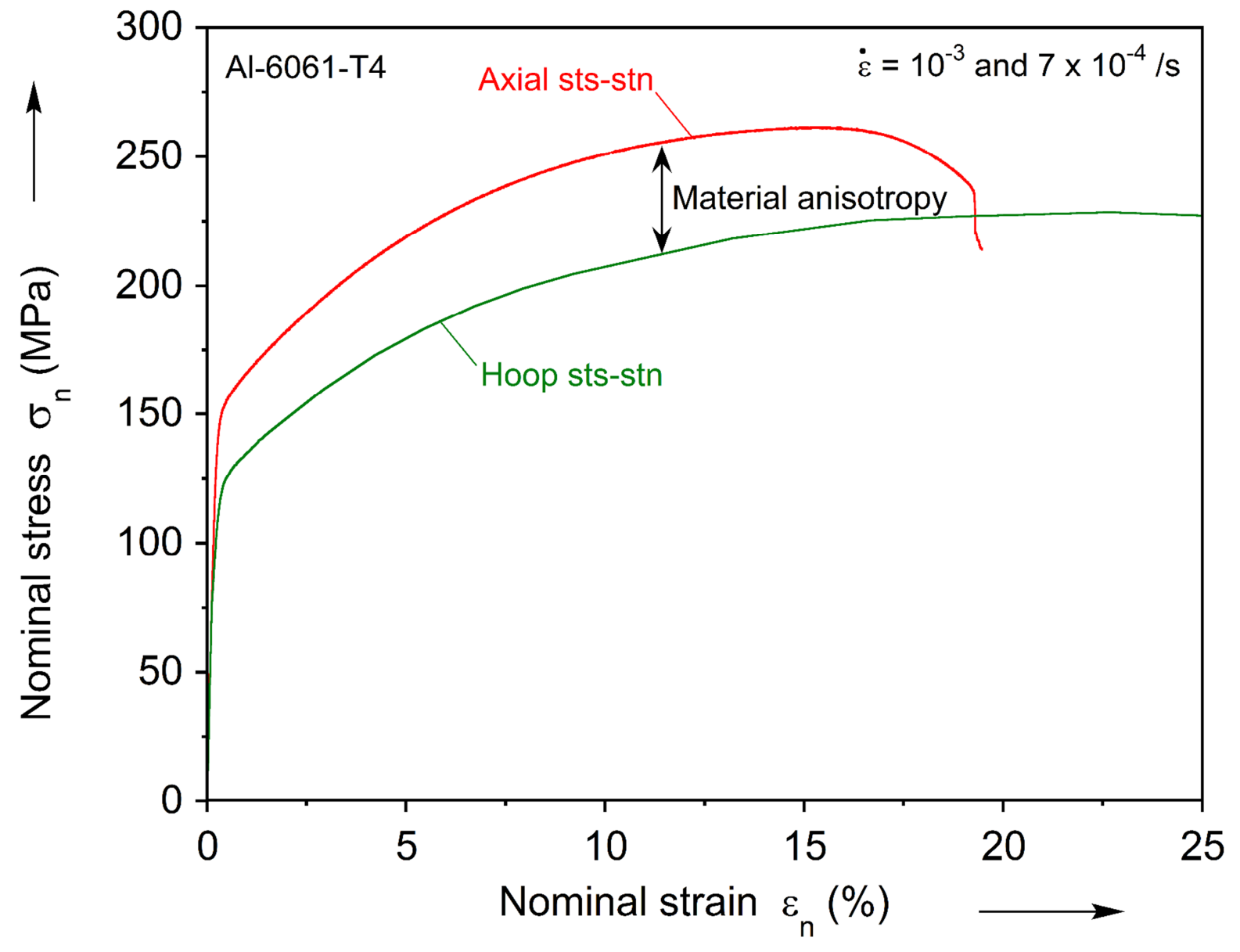

F1b 


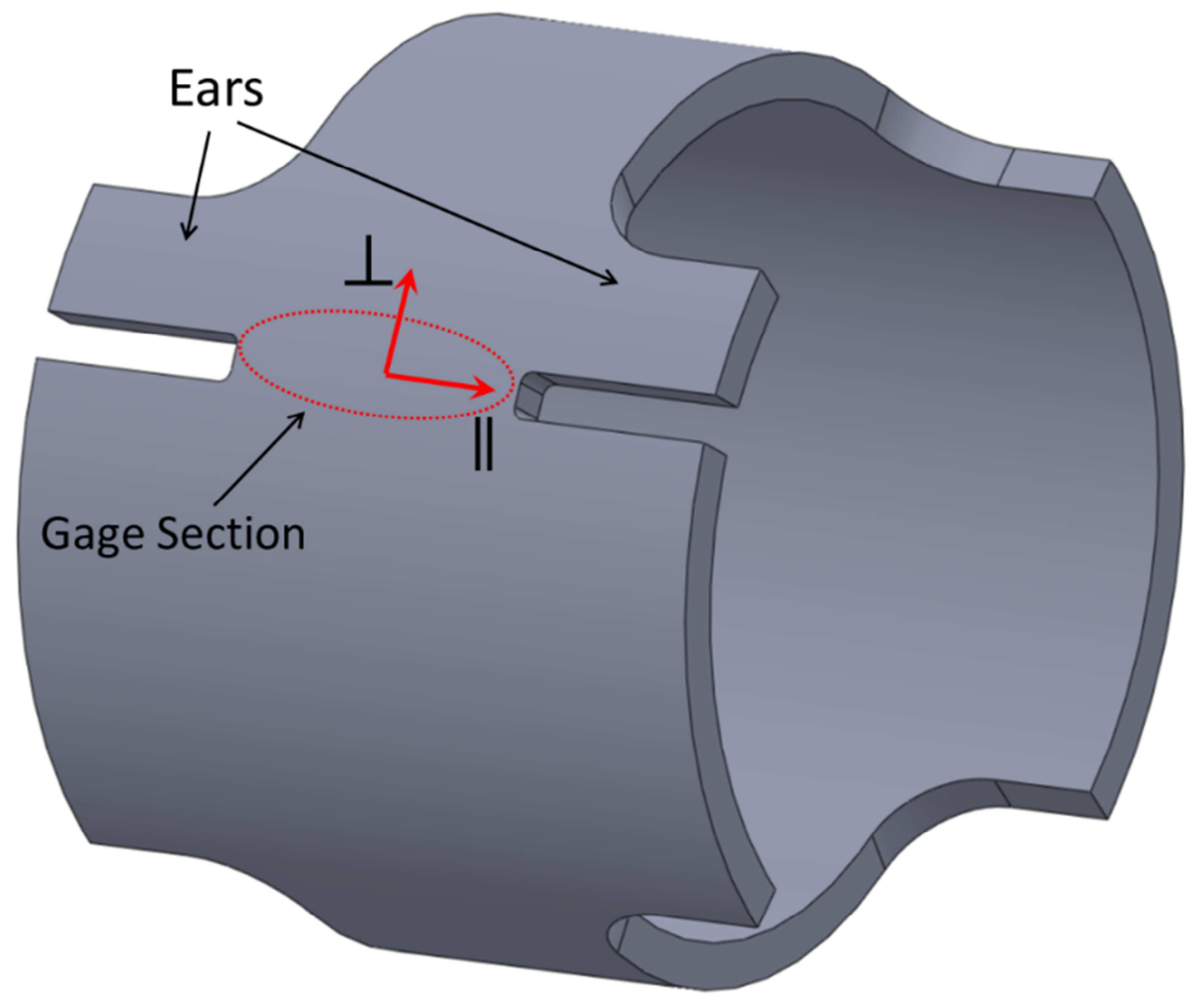

F2 


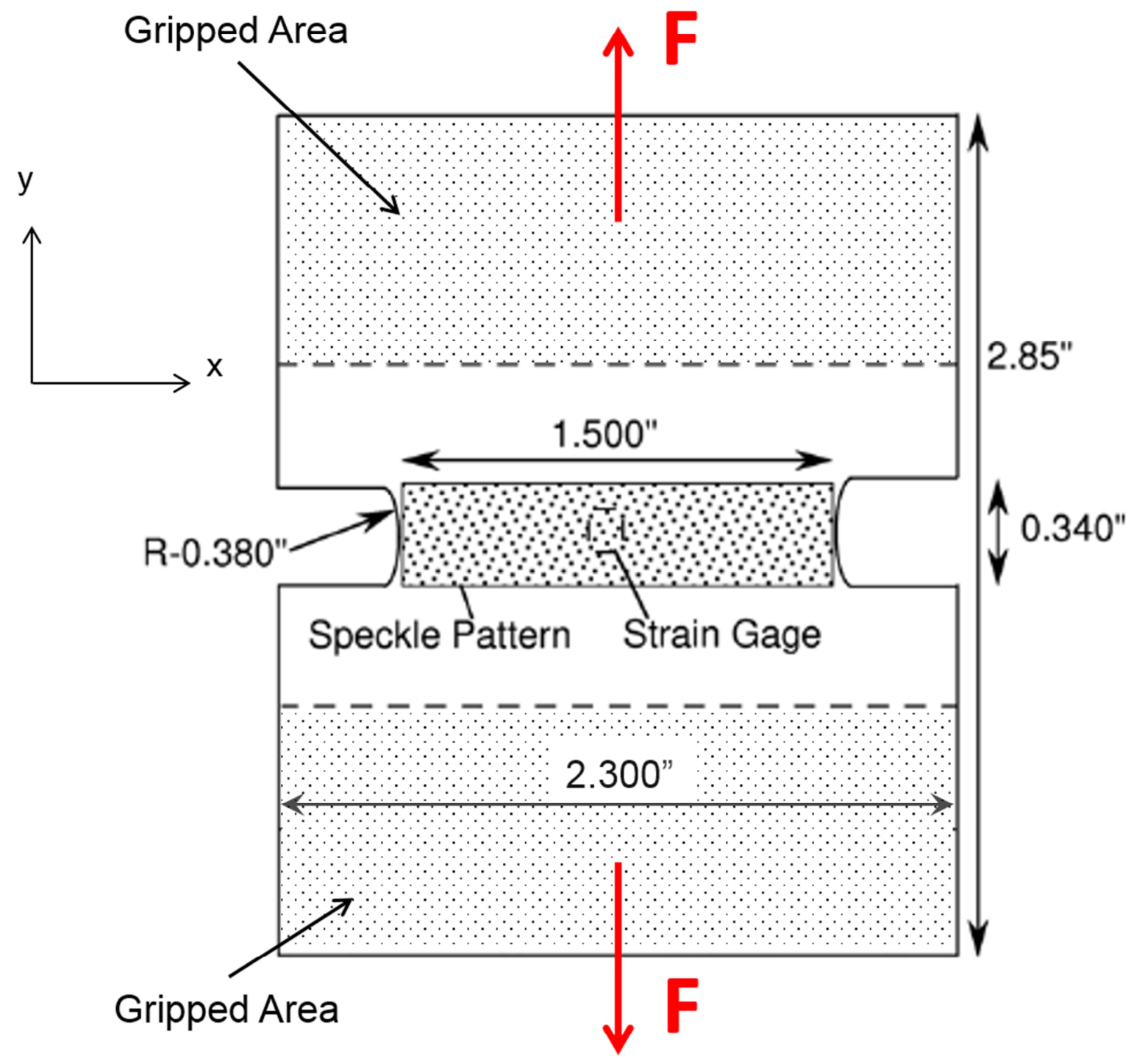

F3 


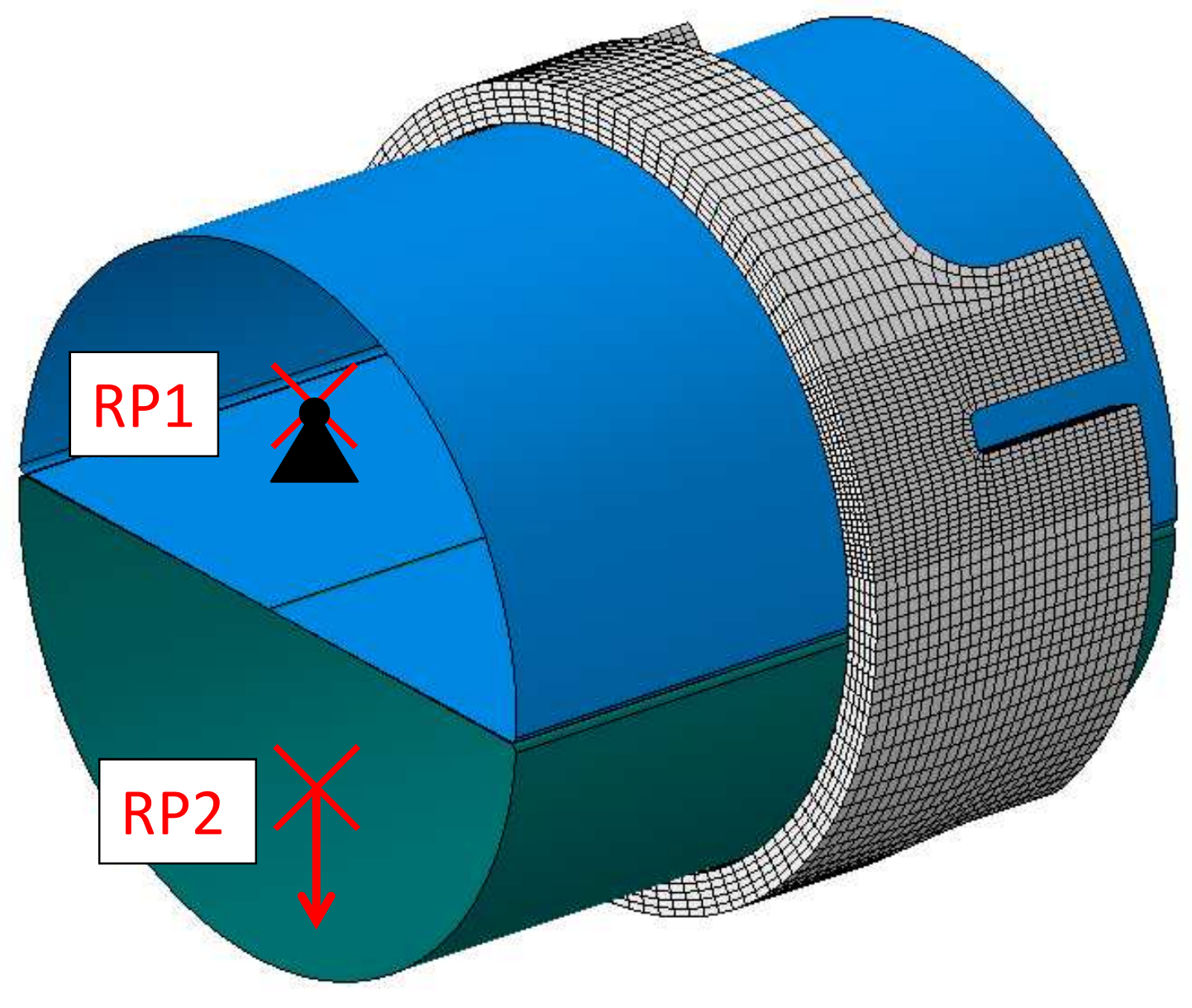

F4a 


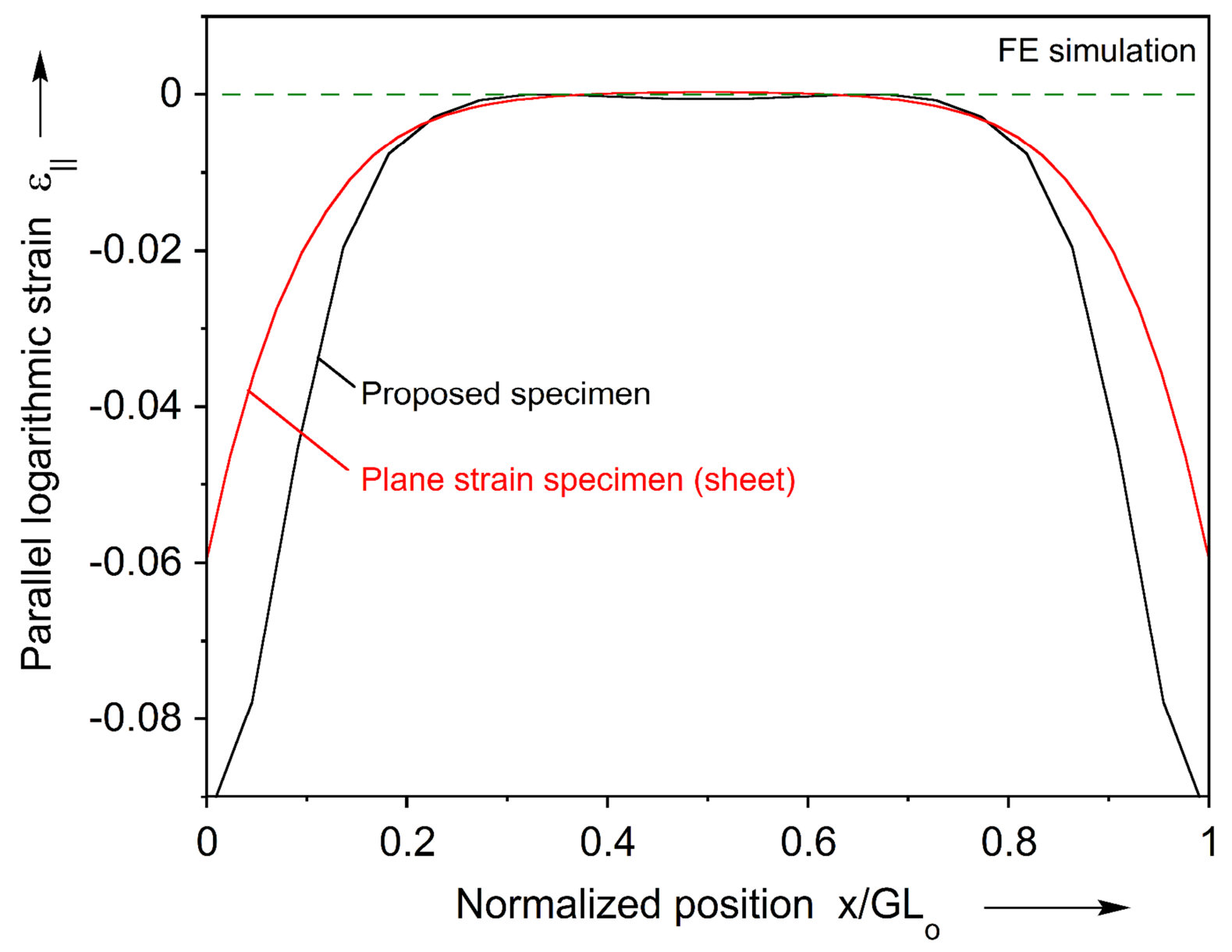

F4b 


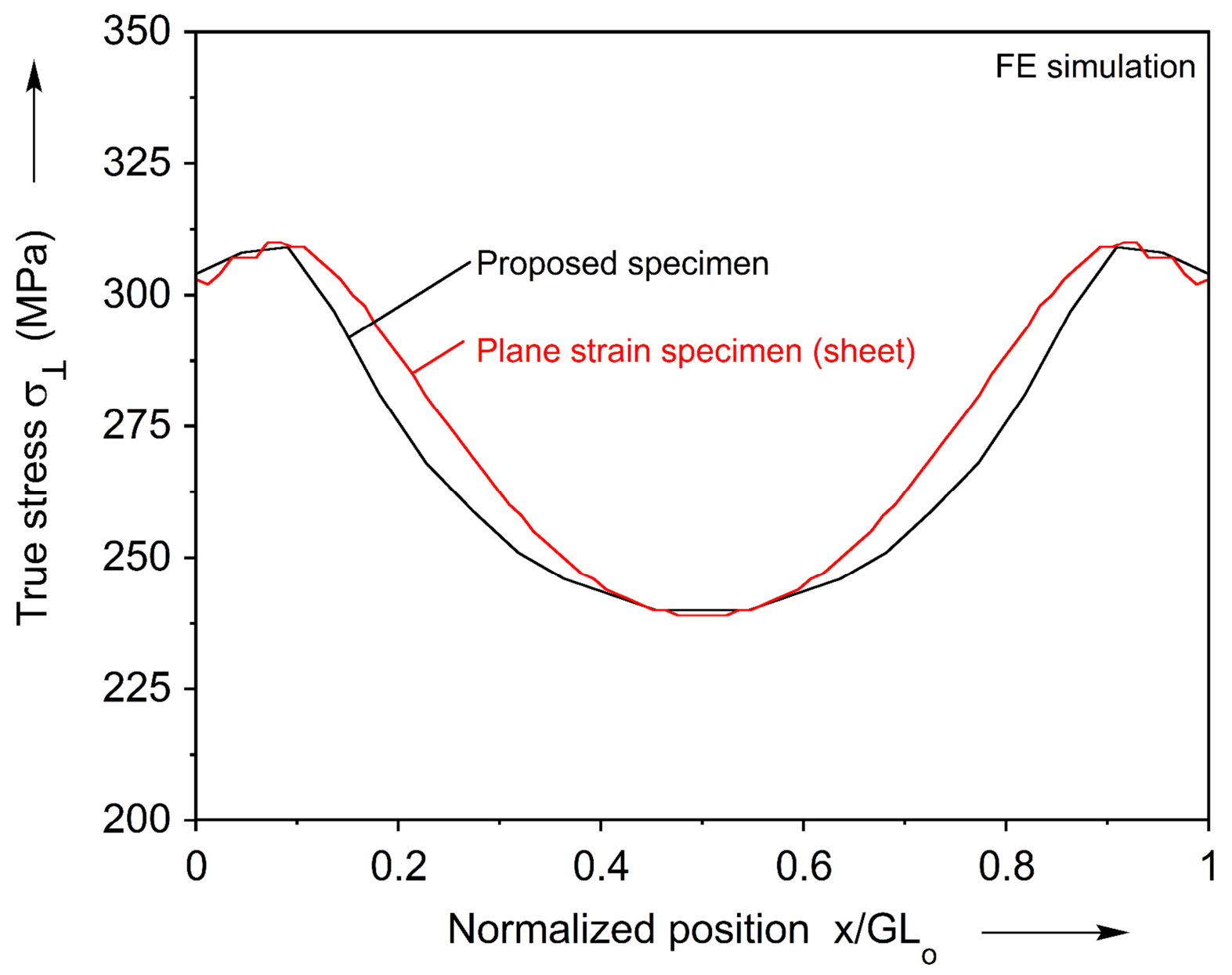

F4c 


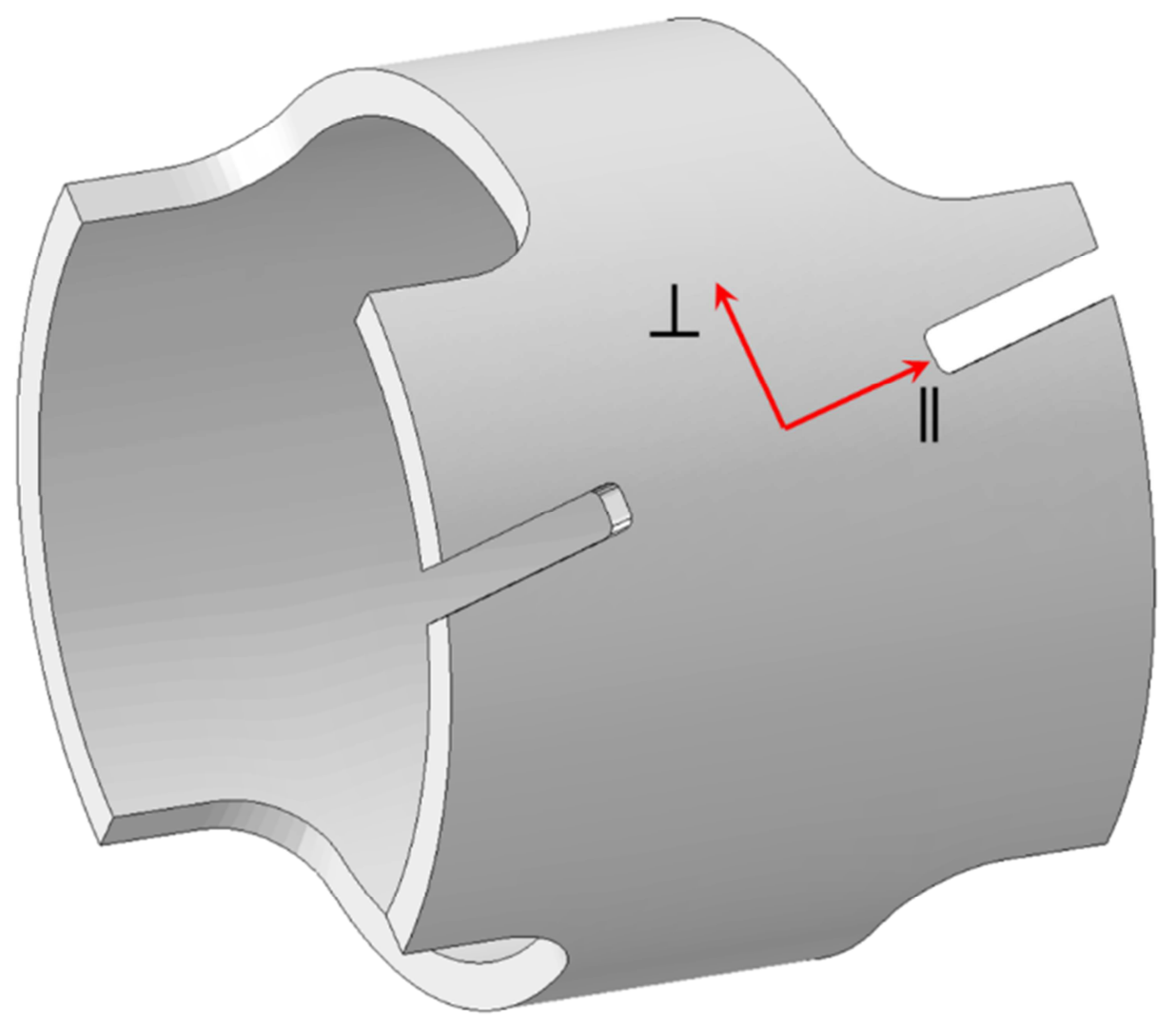

F5a 


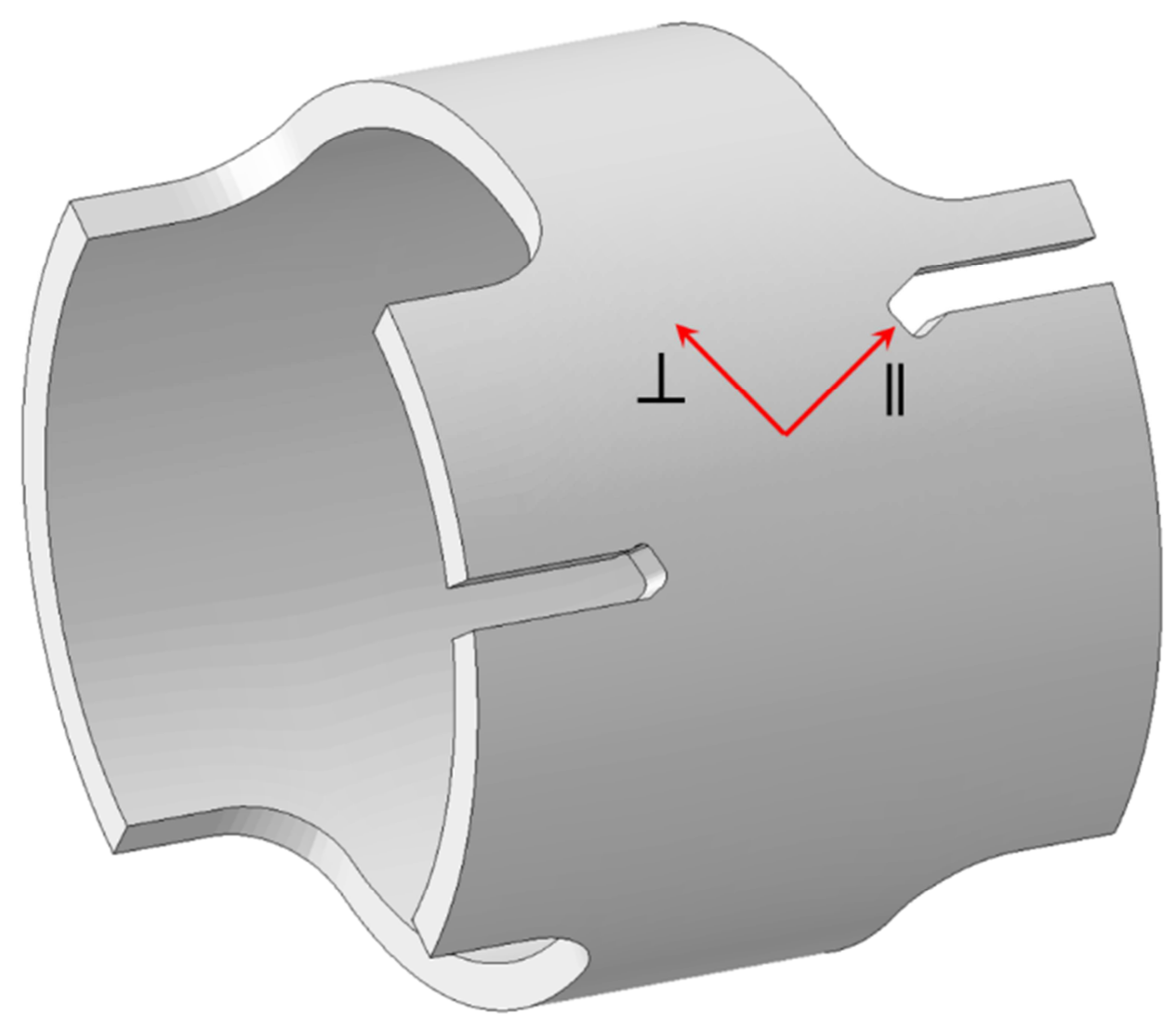

F5b 


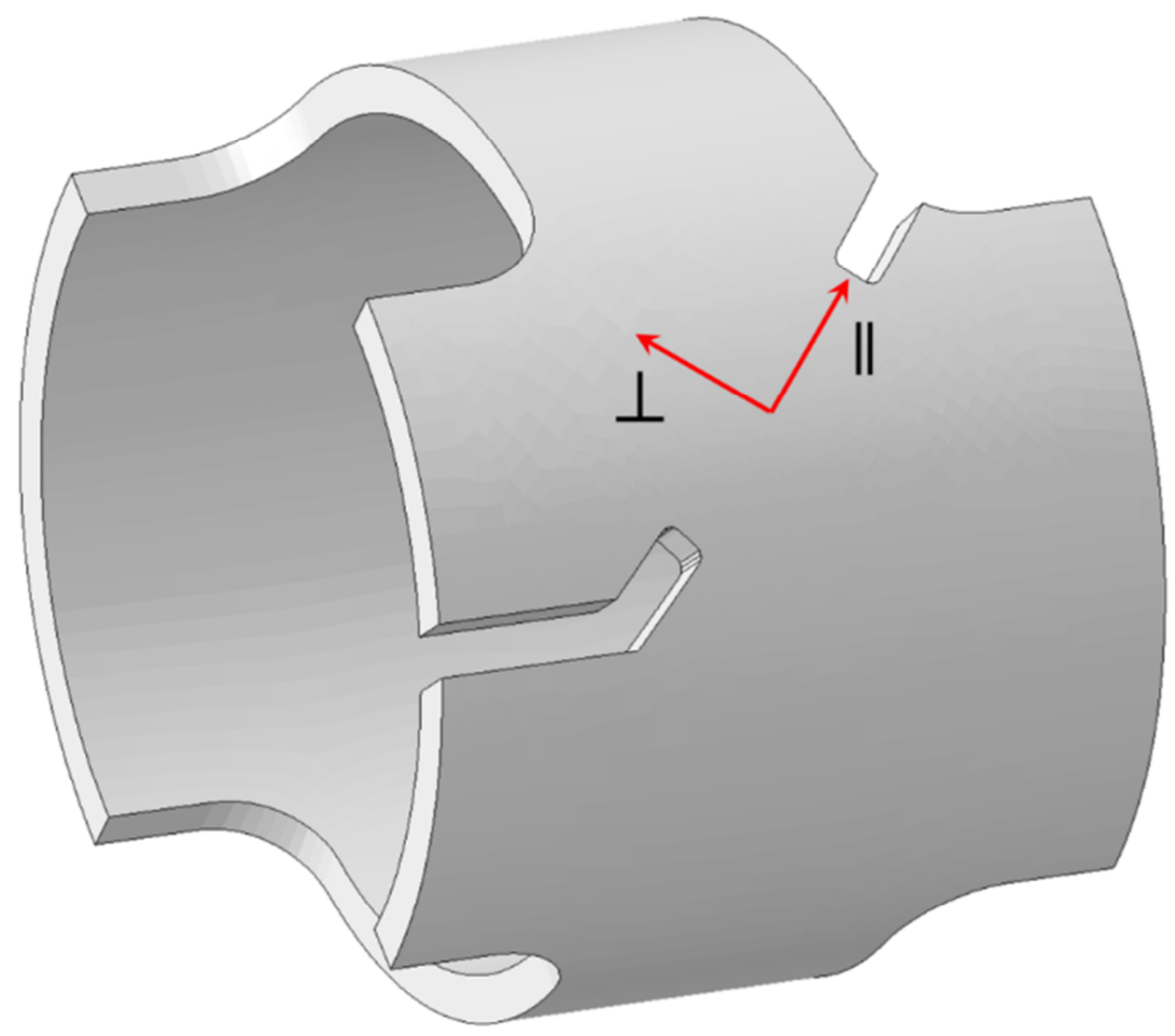

F5c 


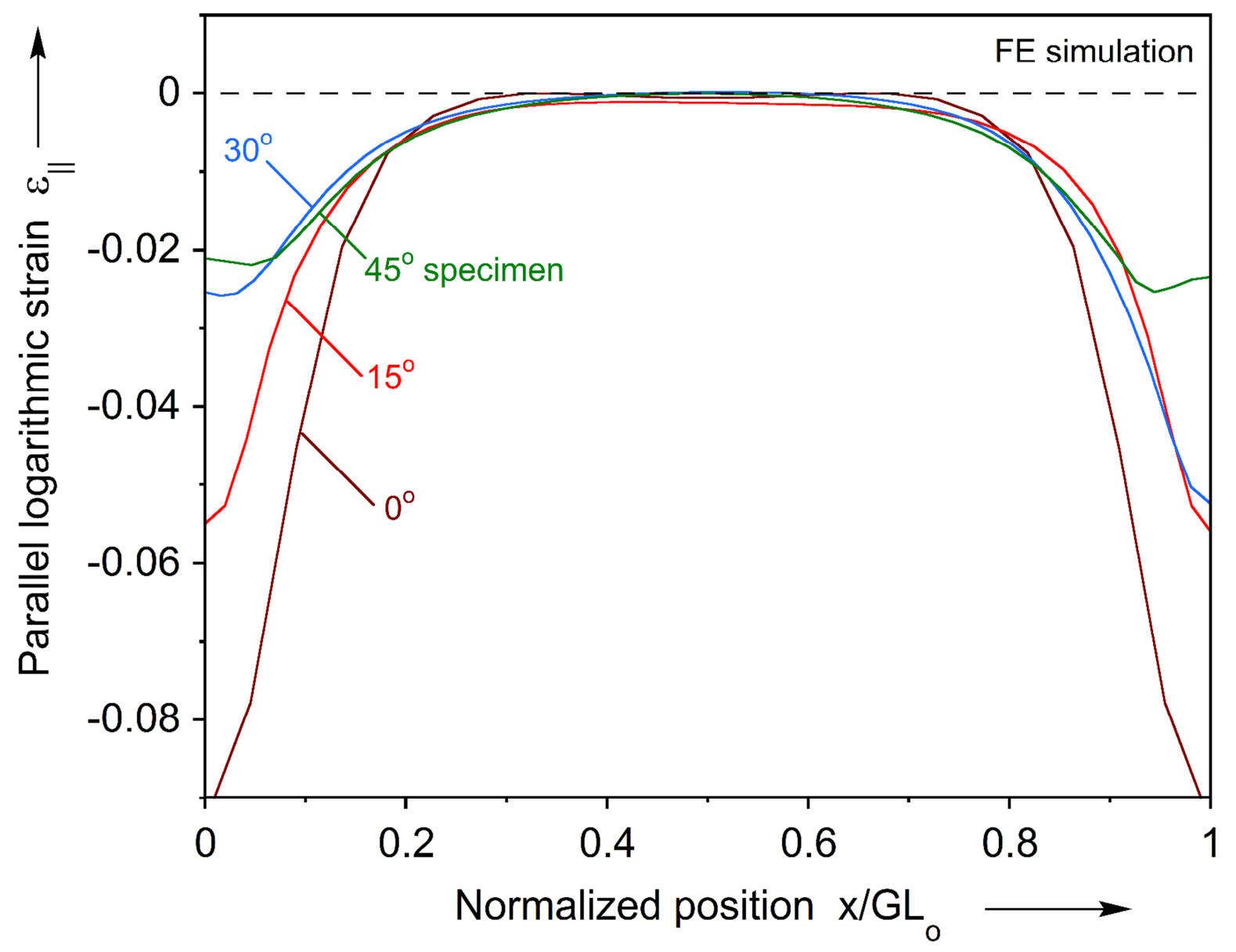

F6 


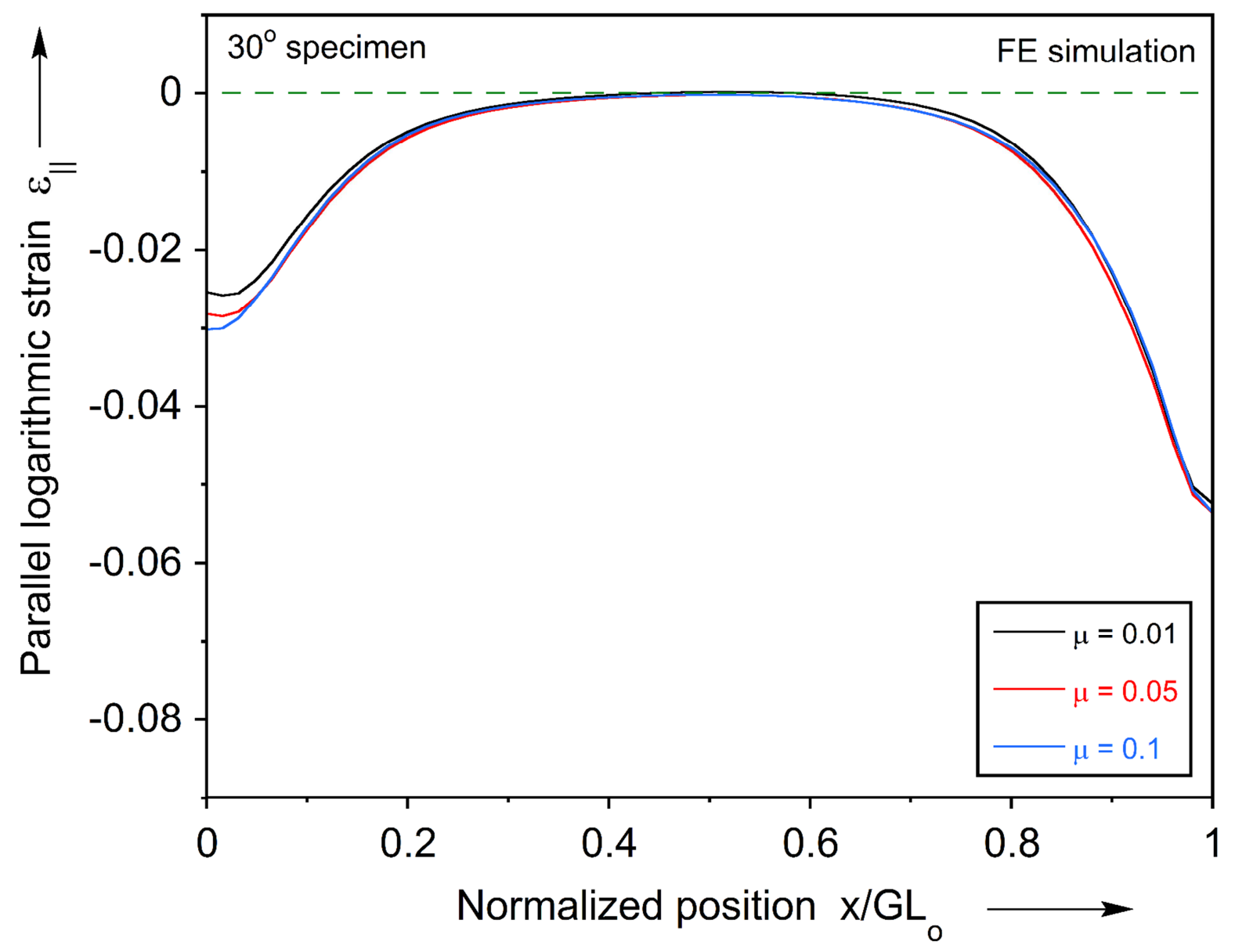

F7 


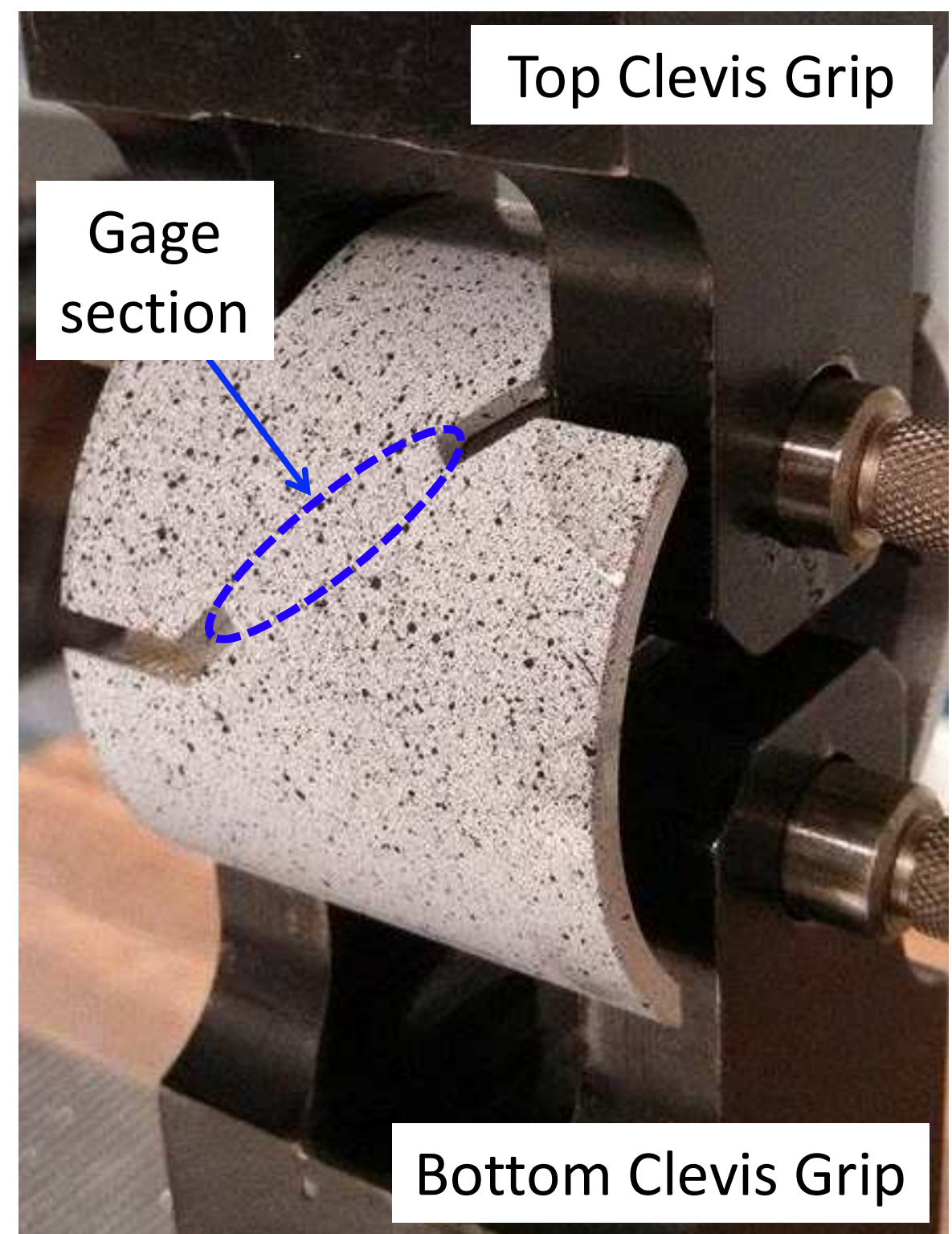

F8 


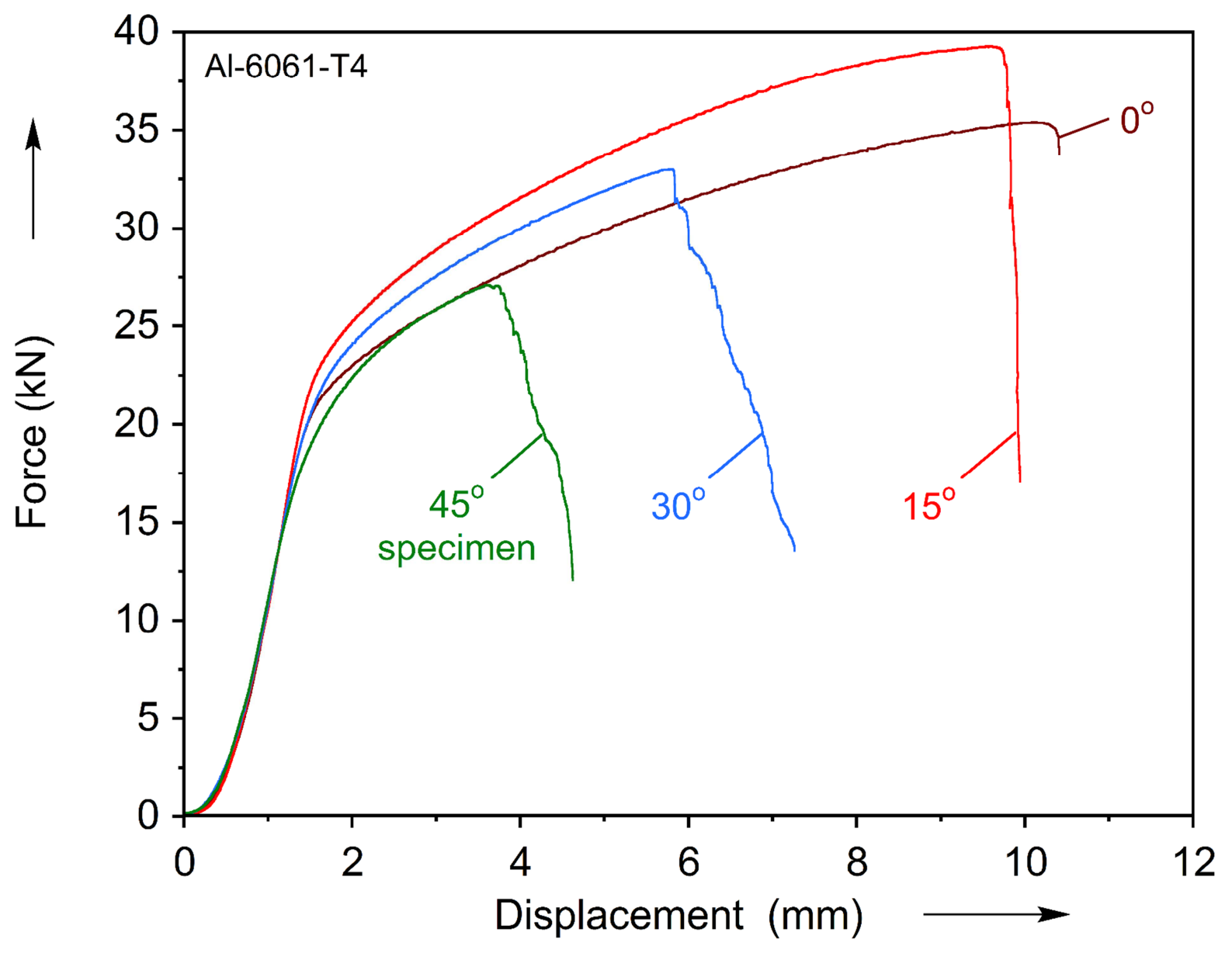

F9 


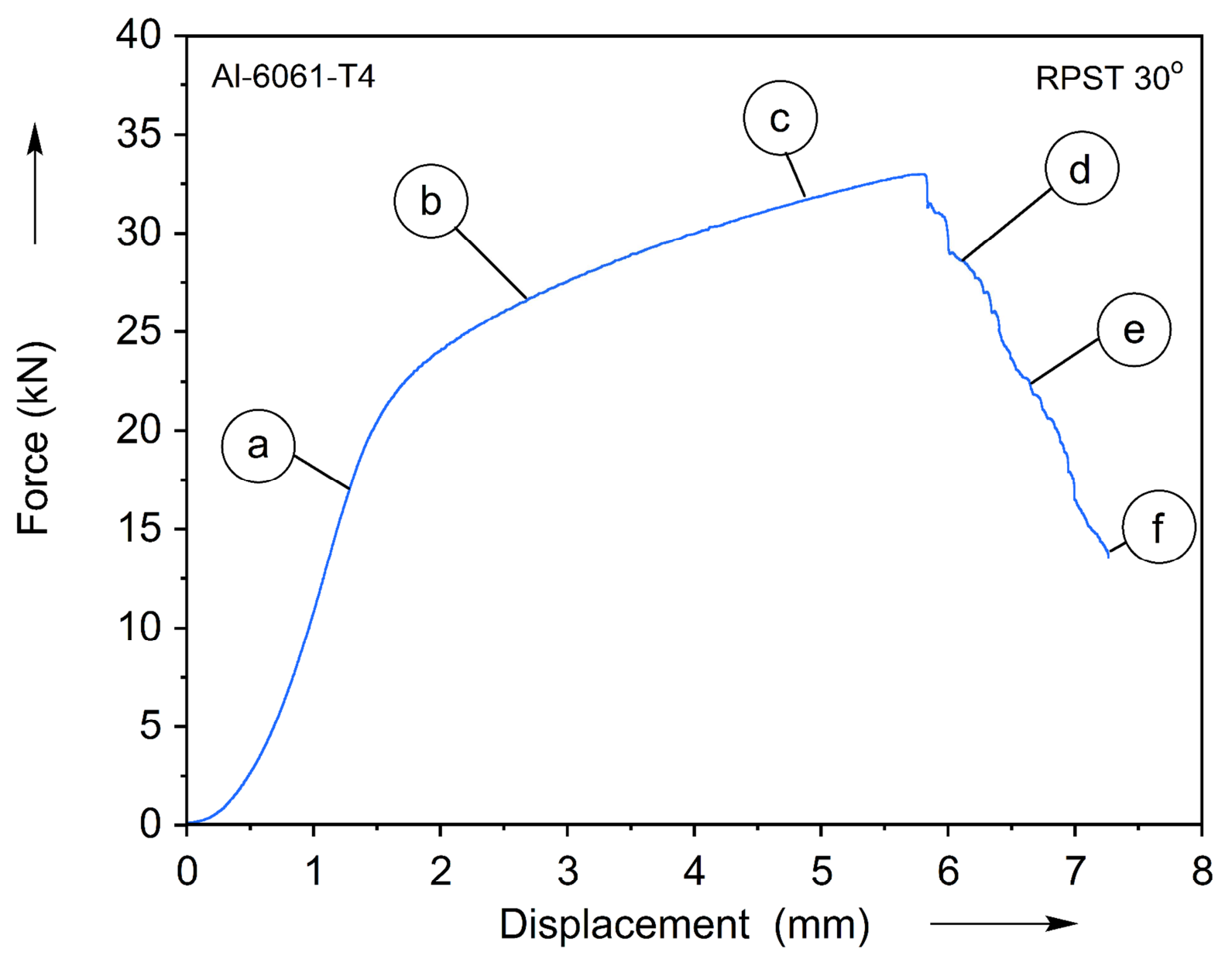

F10 


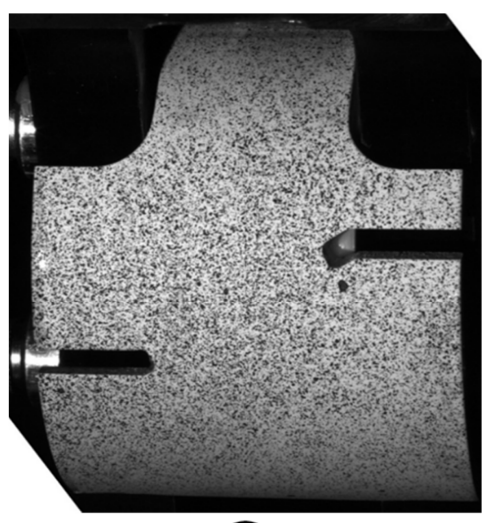

(a)

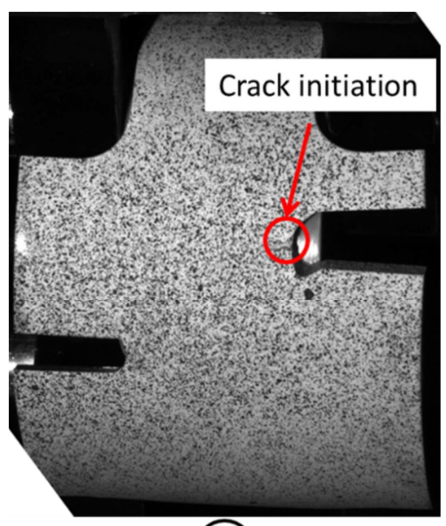

(a)

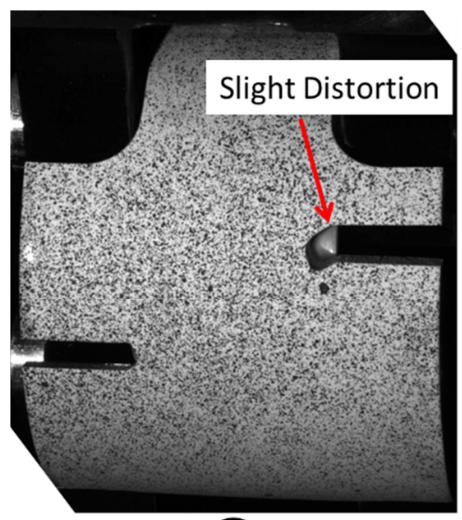

(b)

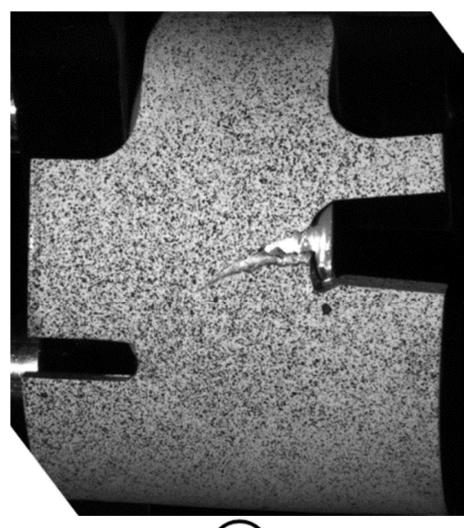

(e)

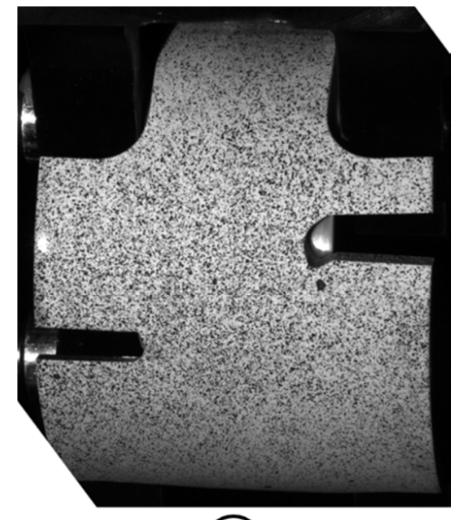

(c)

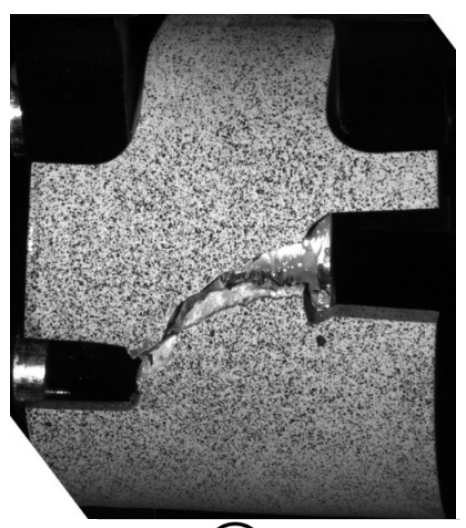

†

F11a-f 


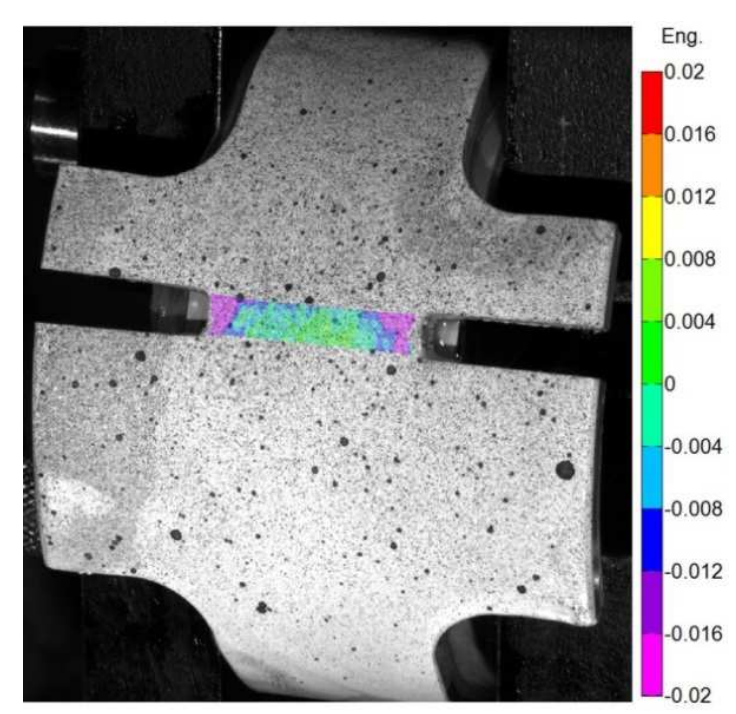

(a)

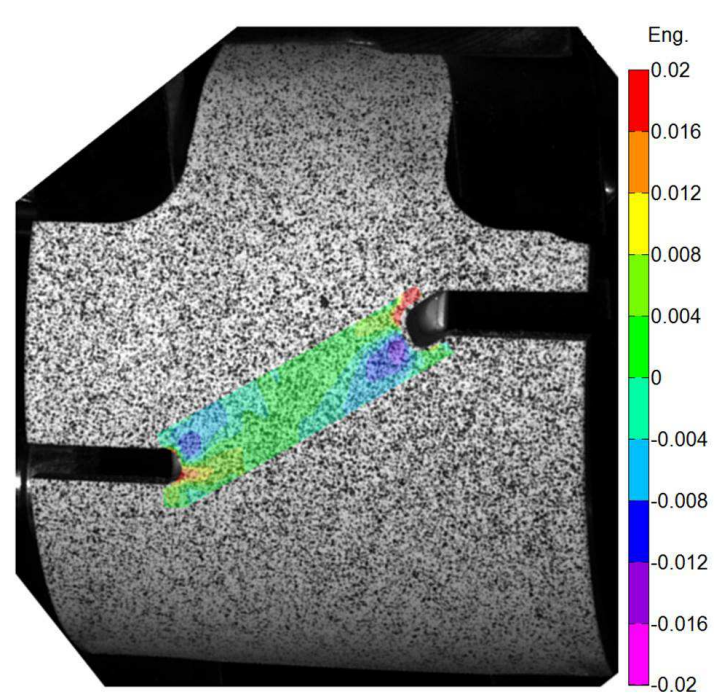

(c)

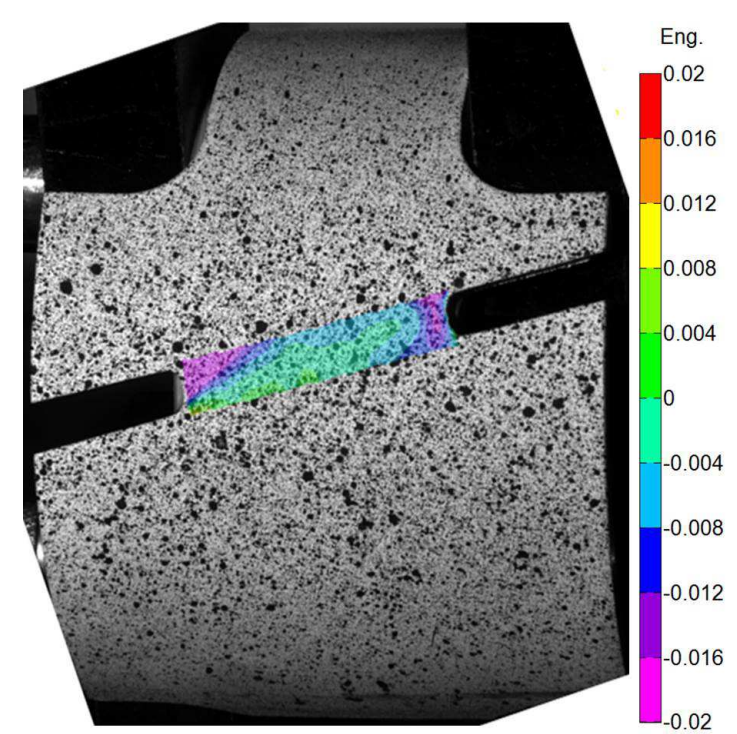

(b)

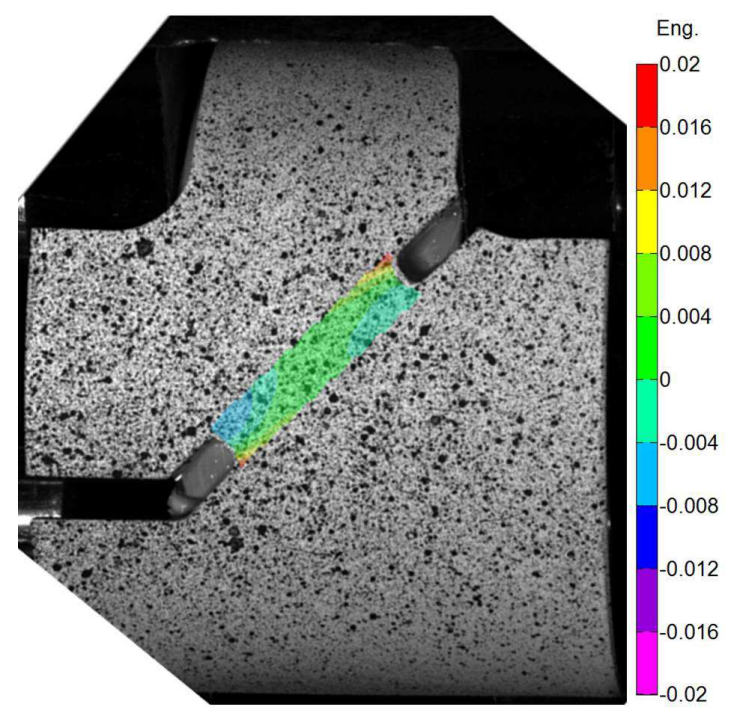

(d)

\section{F12a-d}




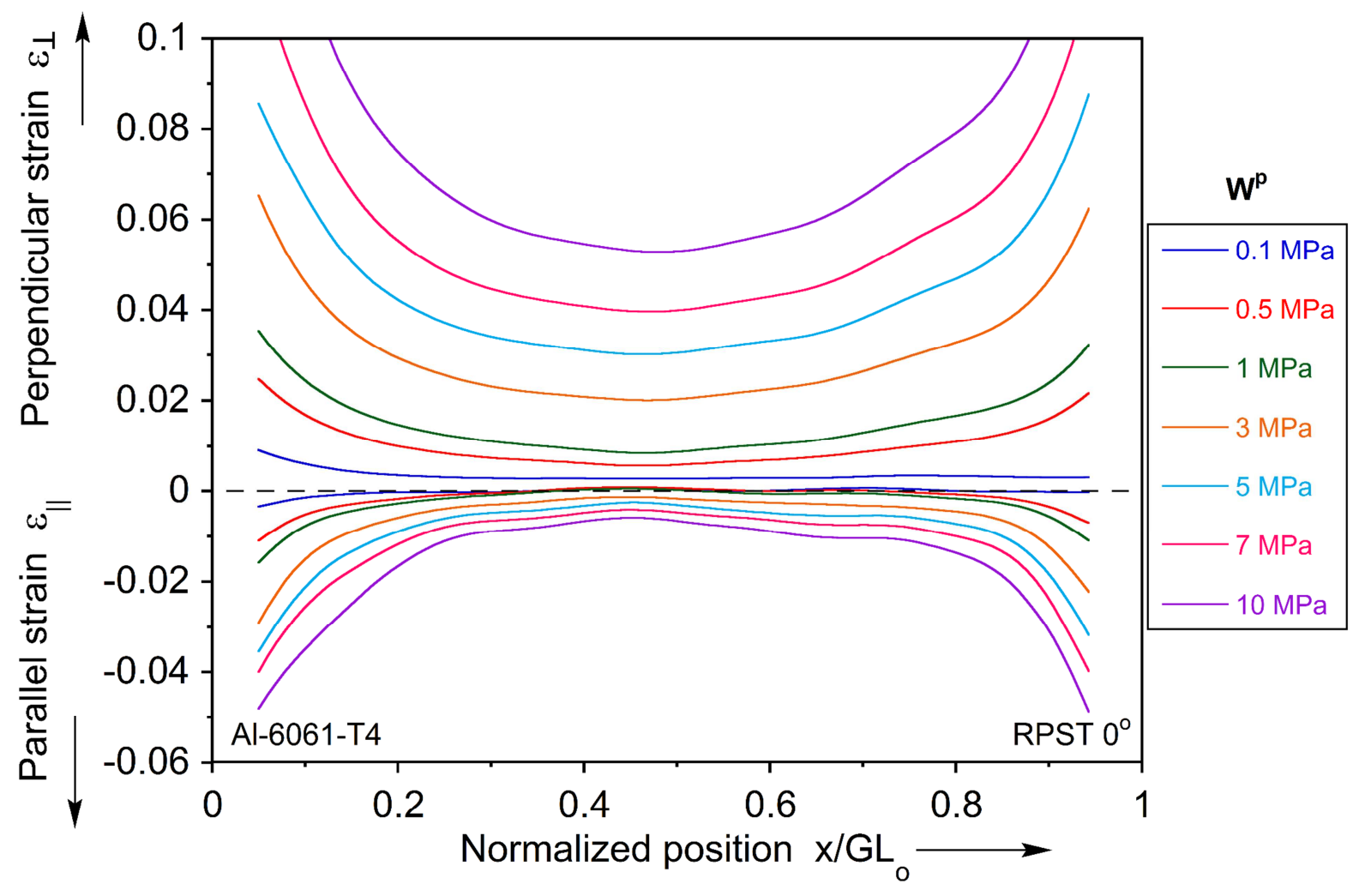

F13 


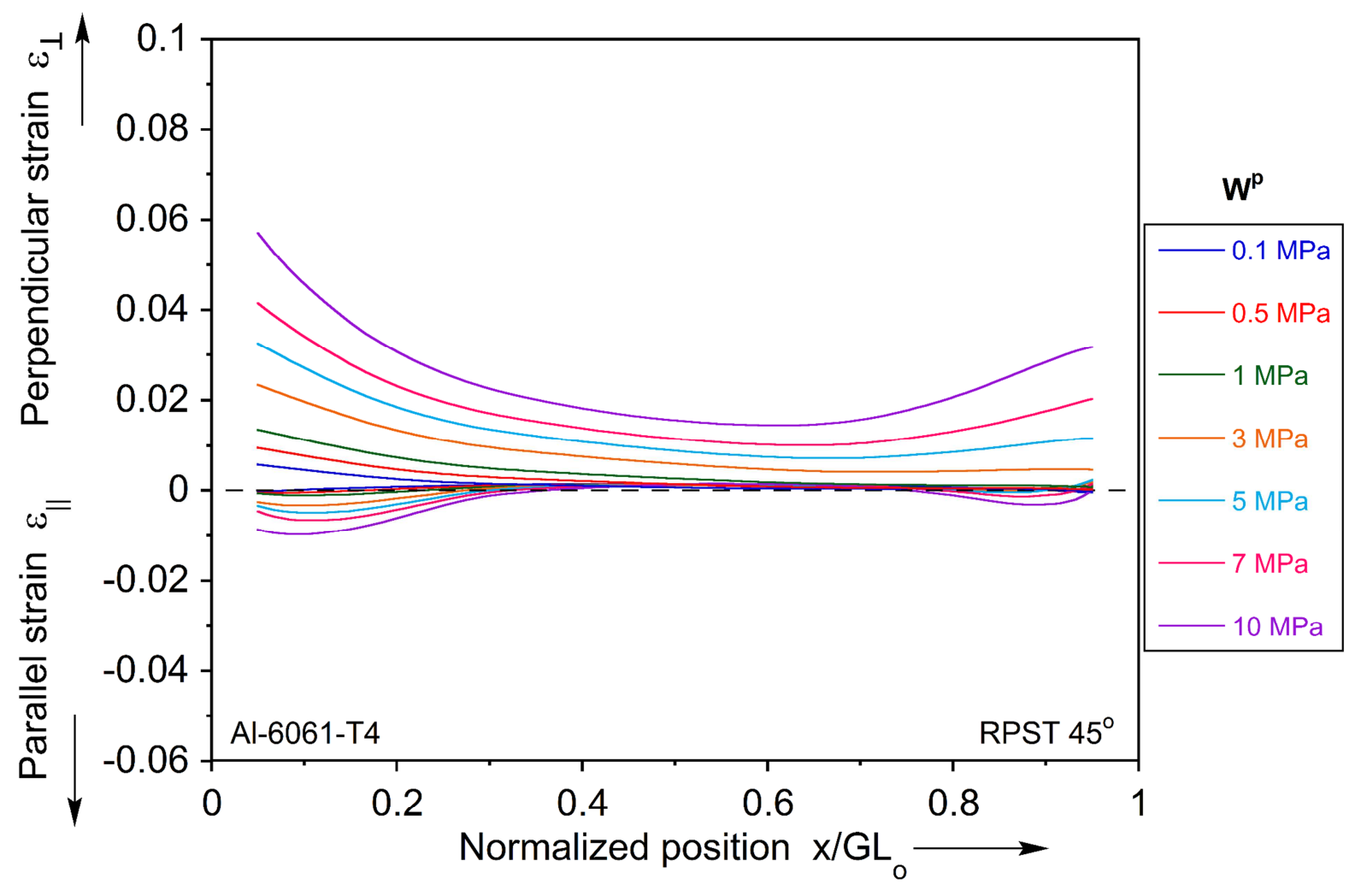

F14a 


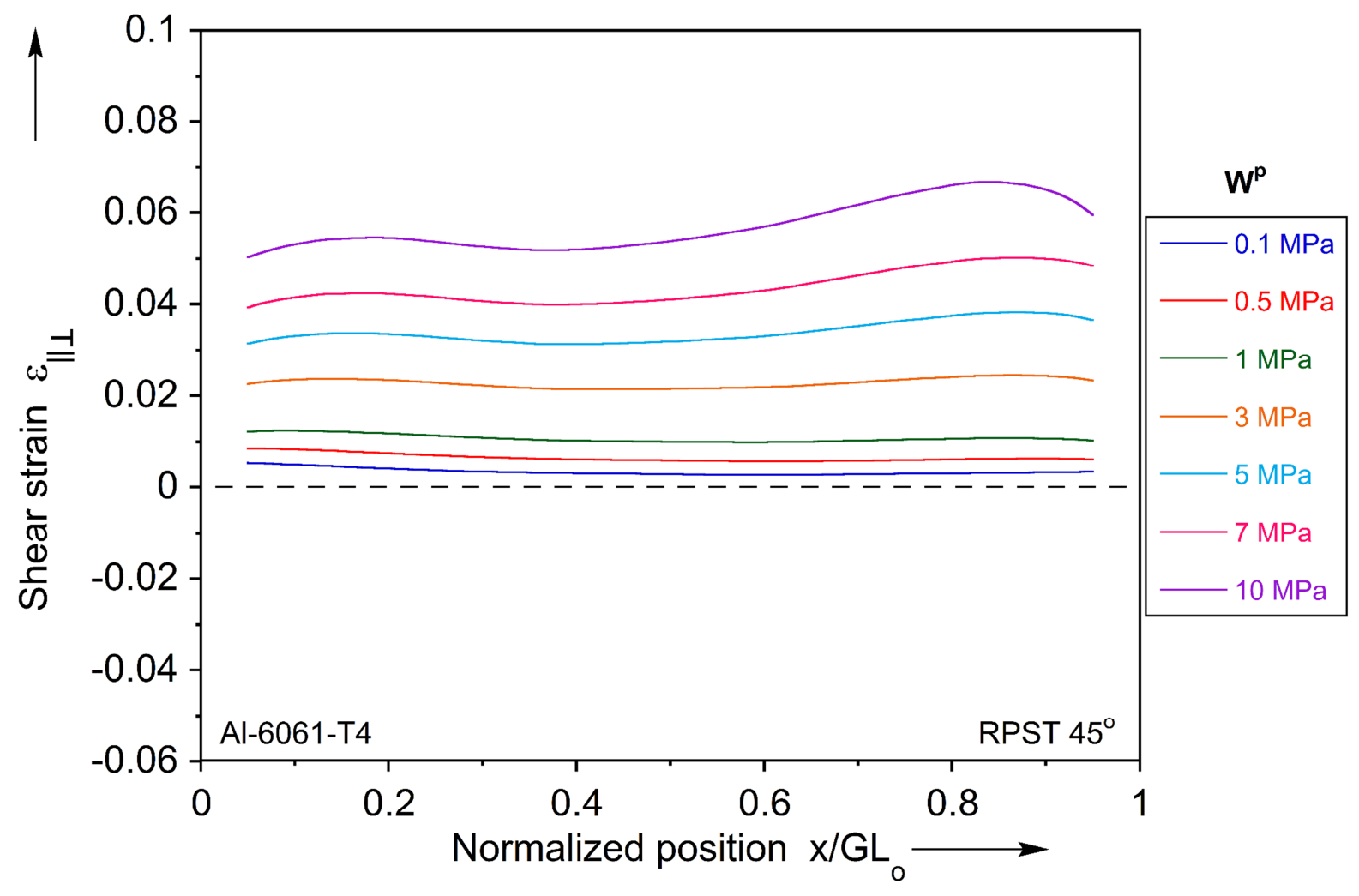

F14b 


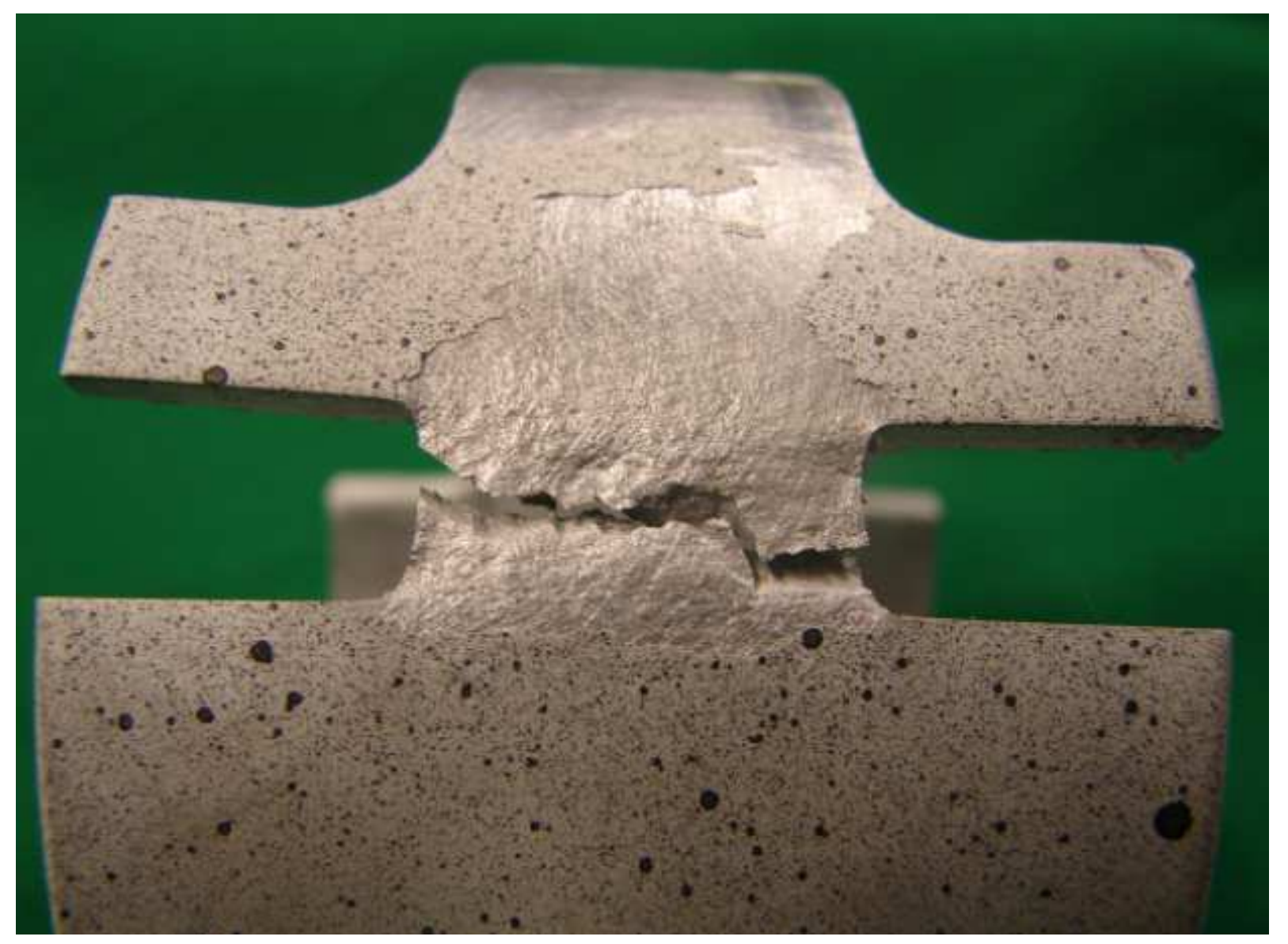

F15 

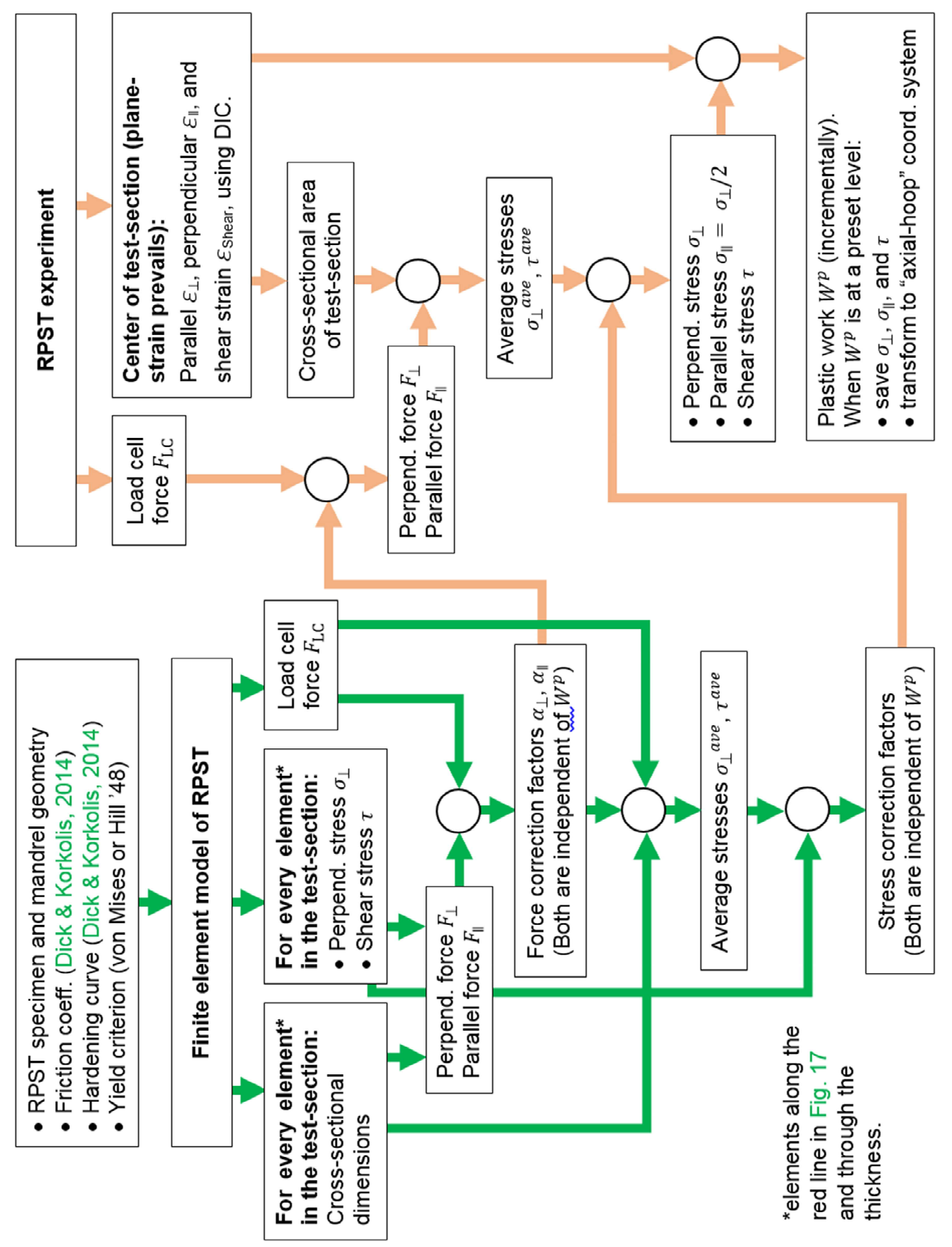

F16 


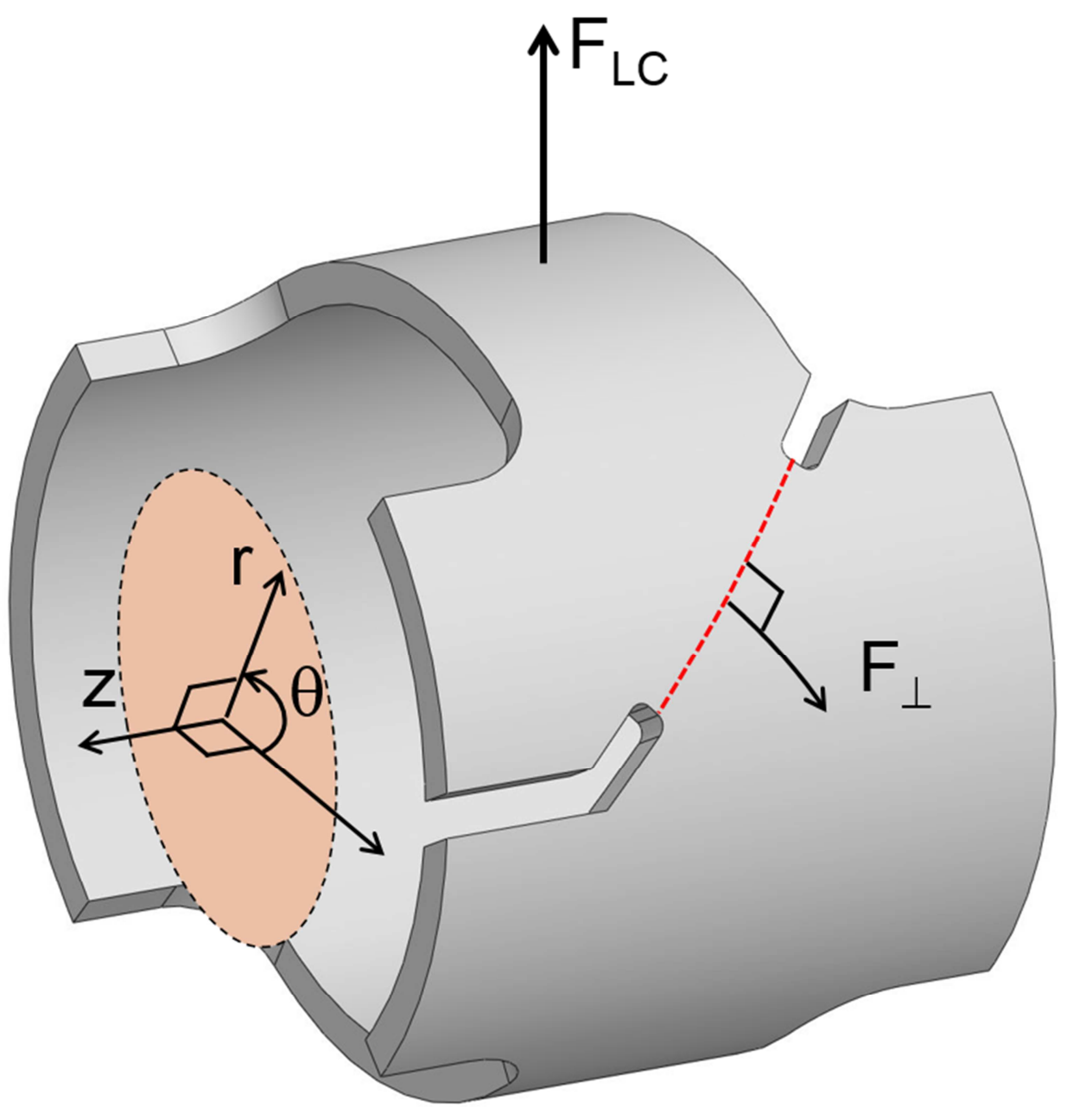

F17a 


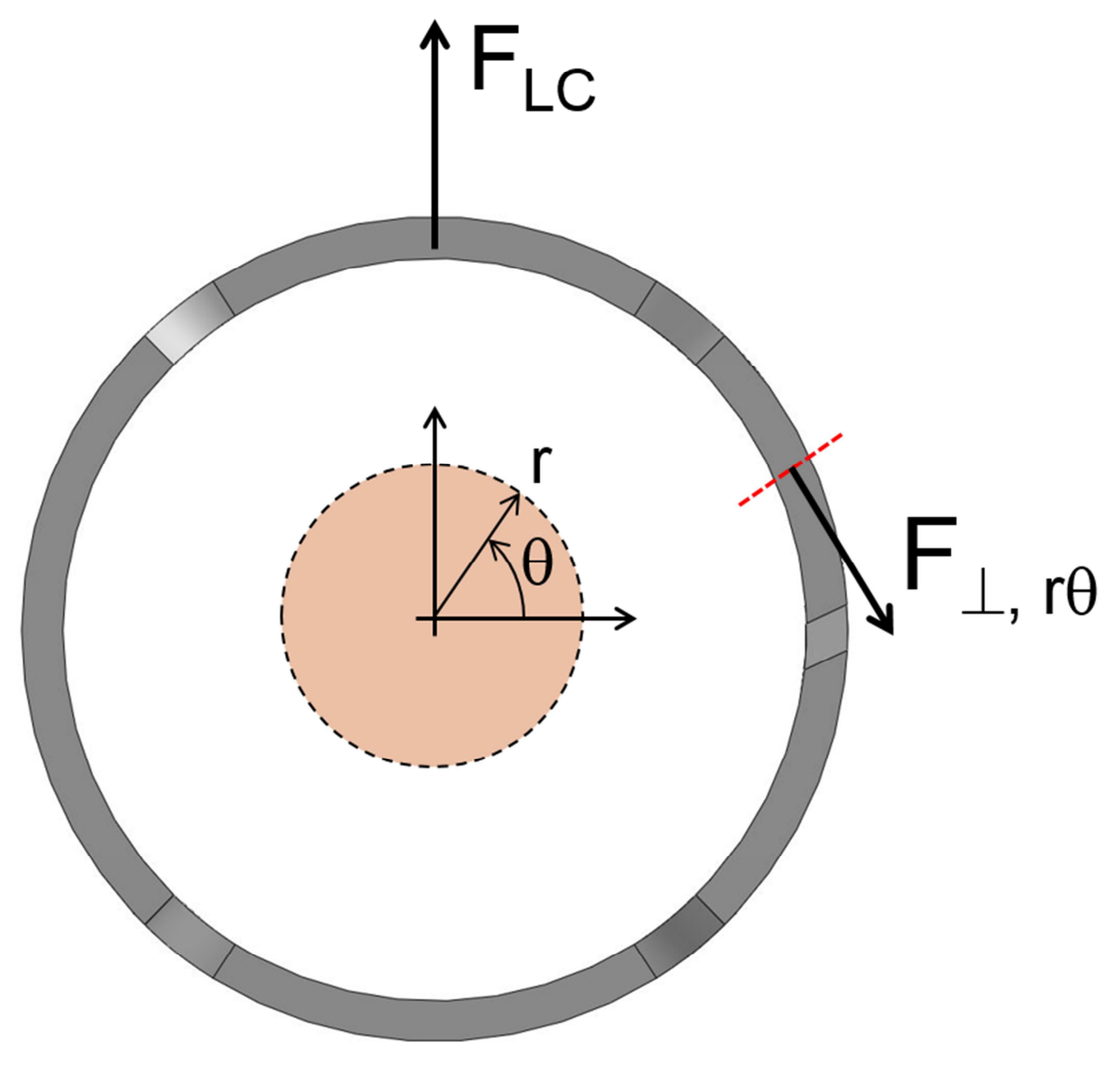

F17b 


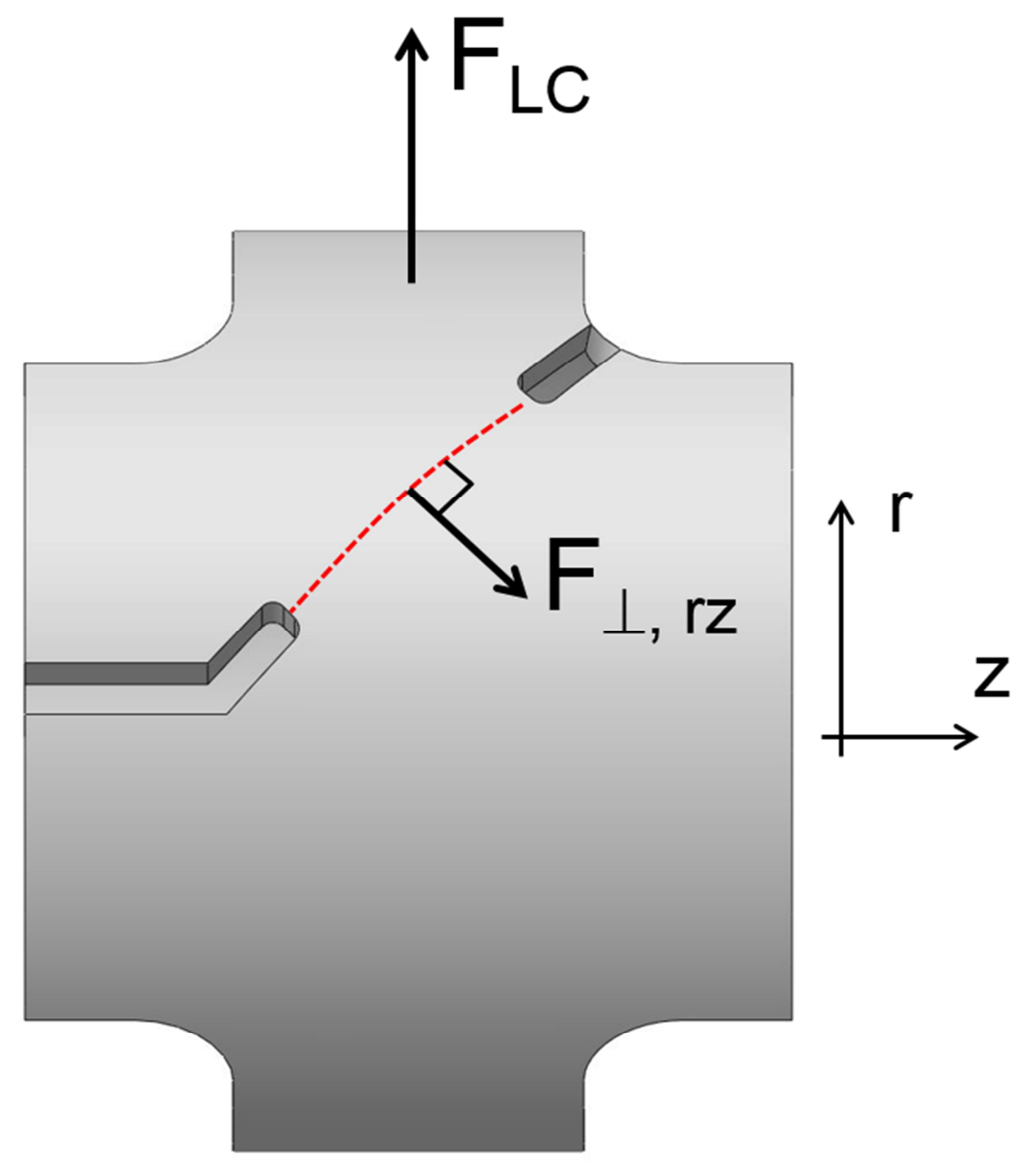

F17c 


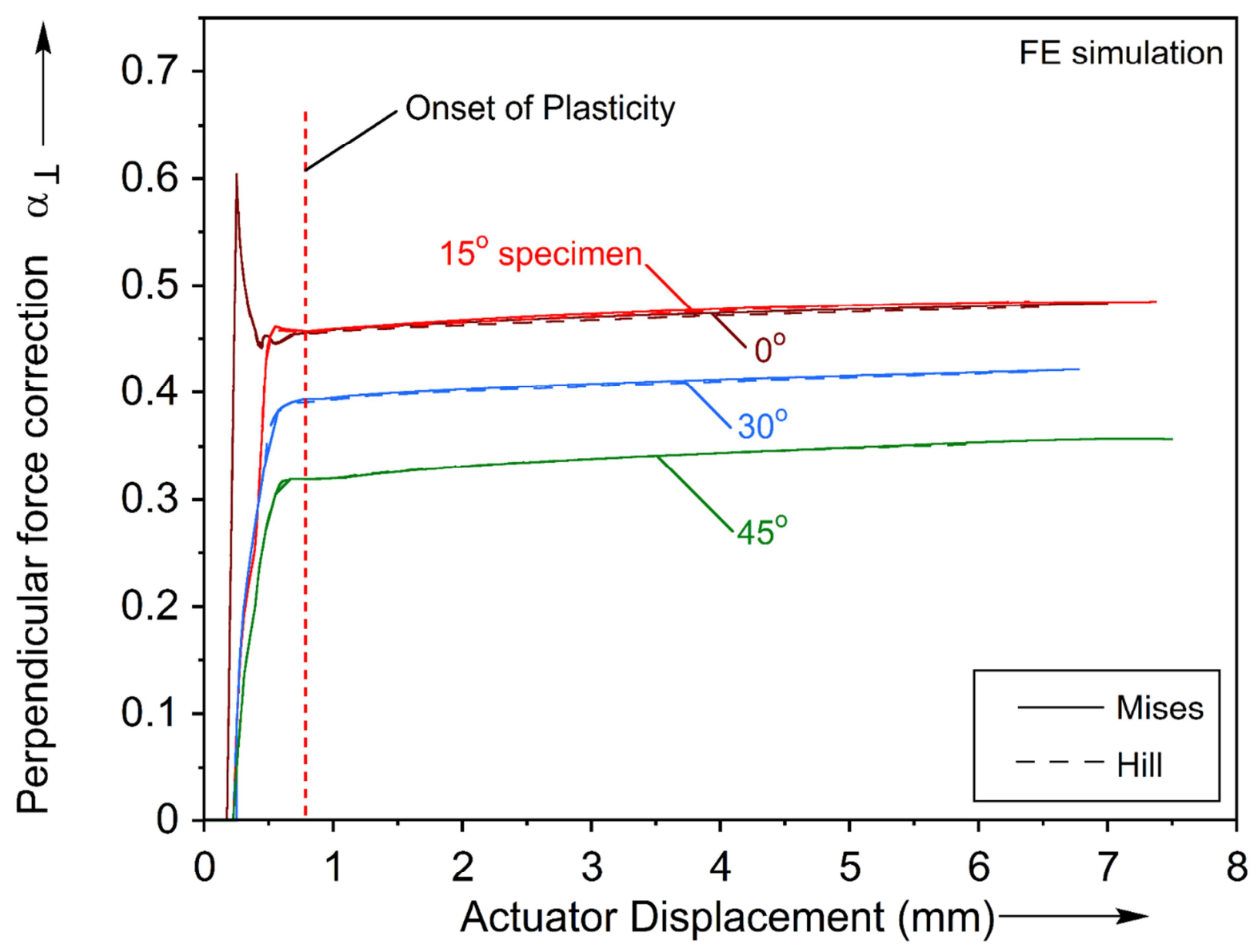

F18a 


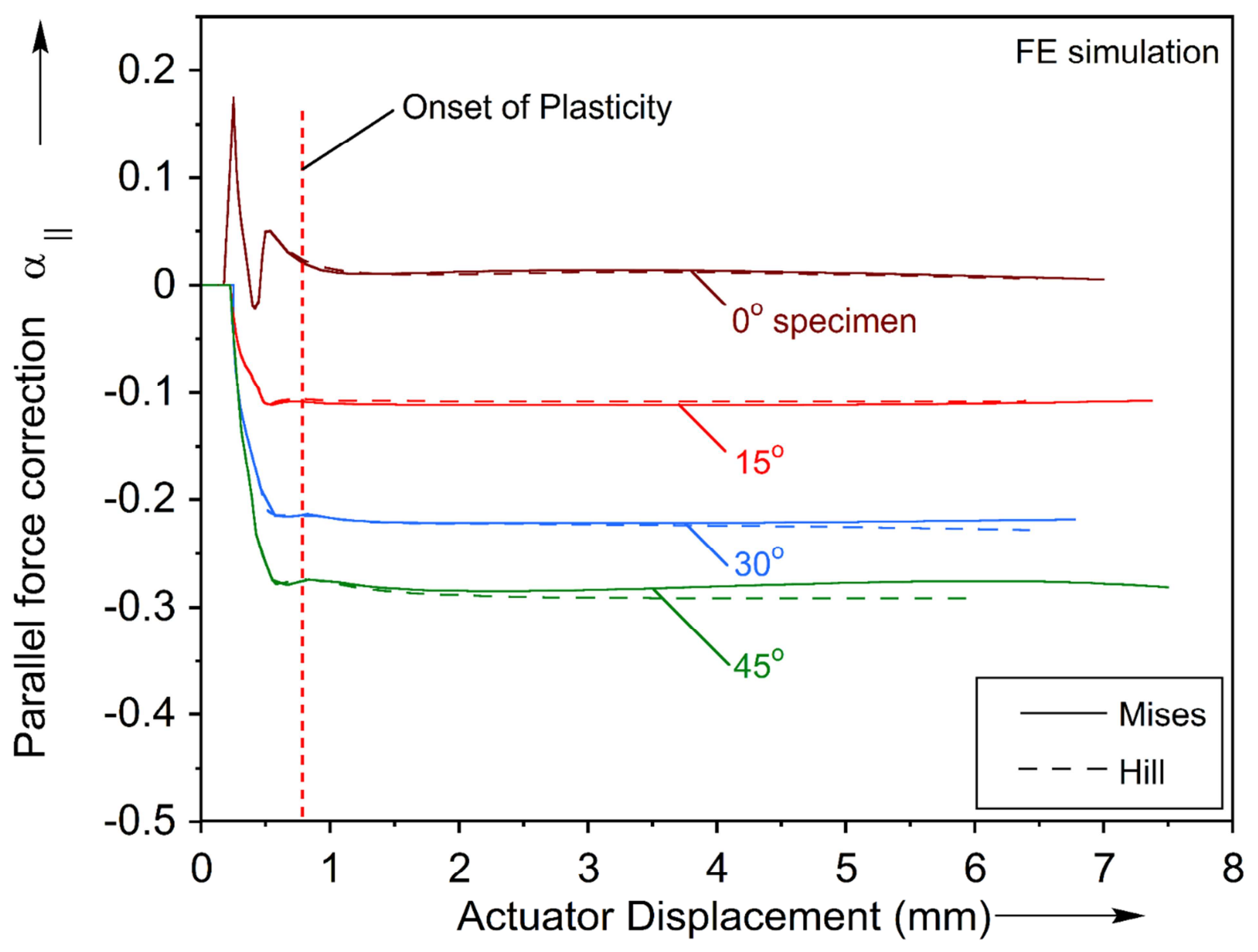

F18b 


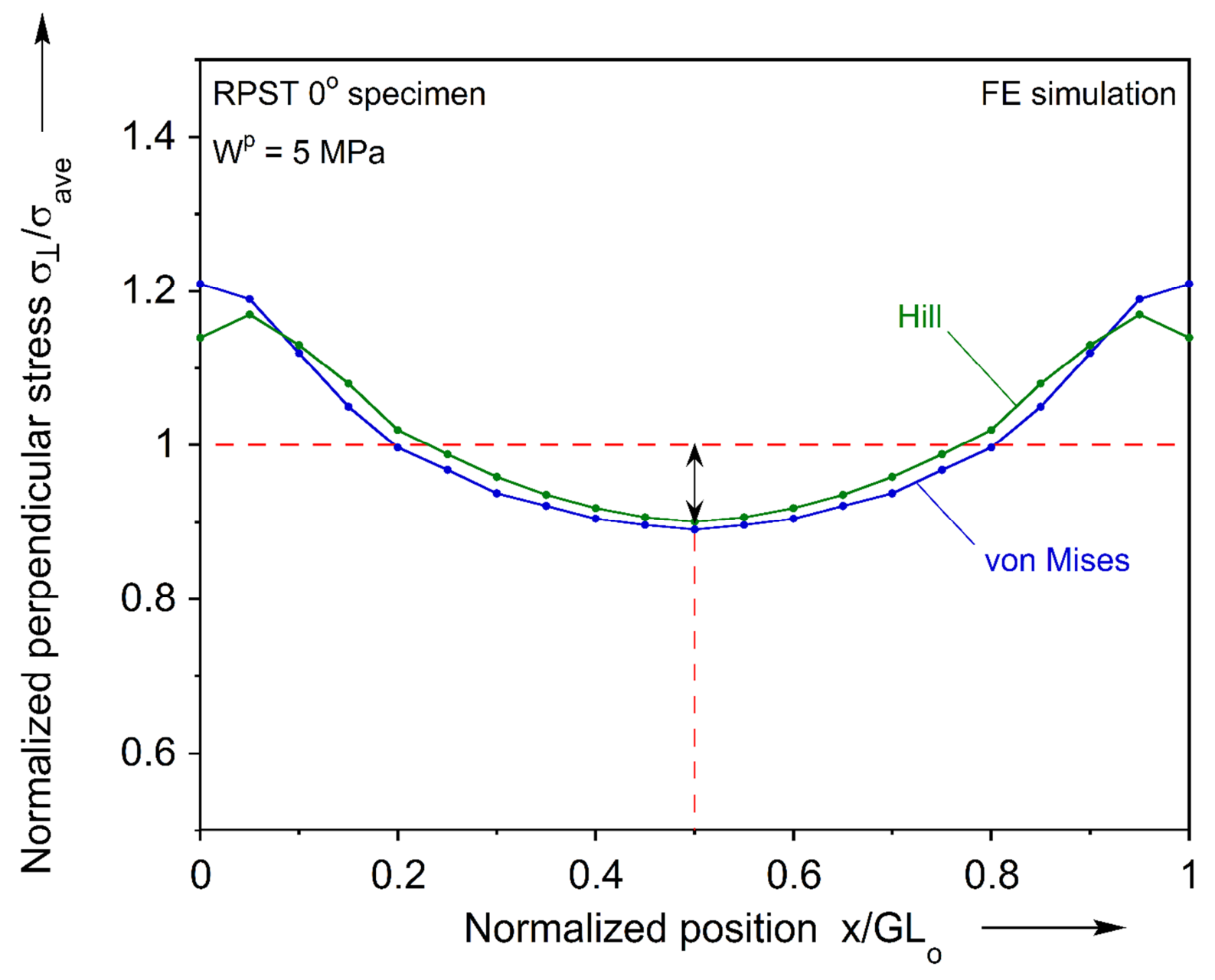

F19a 


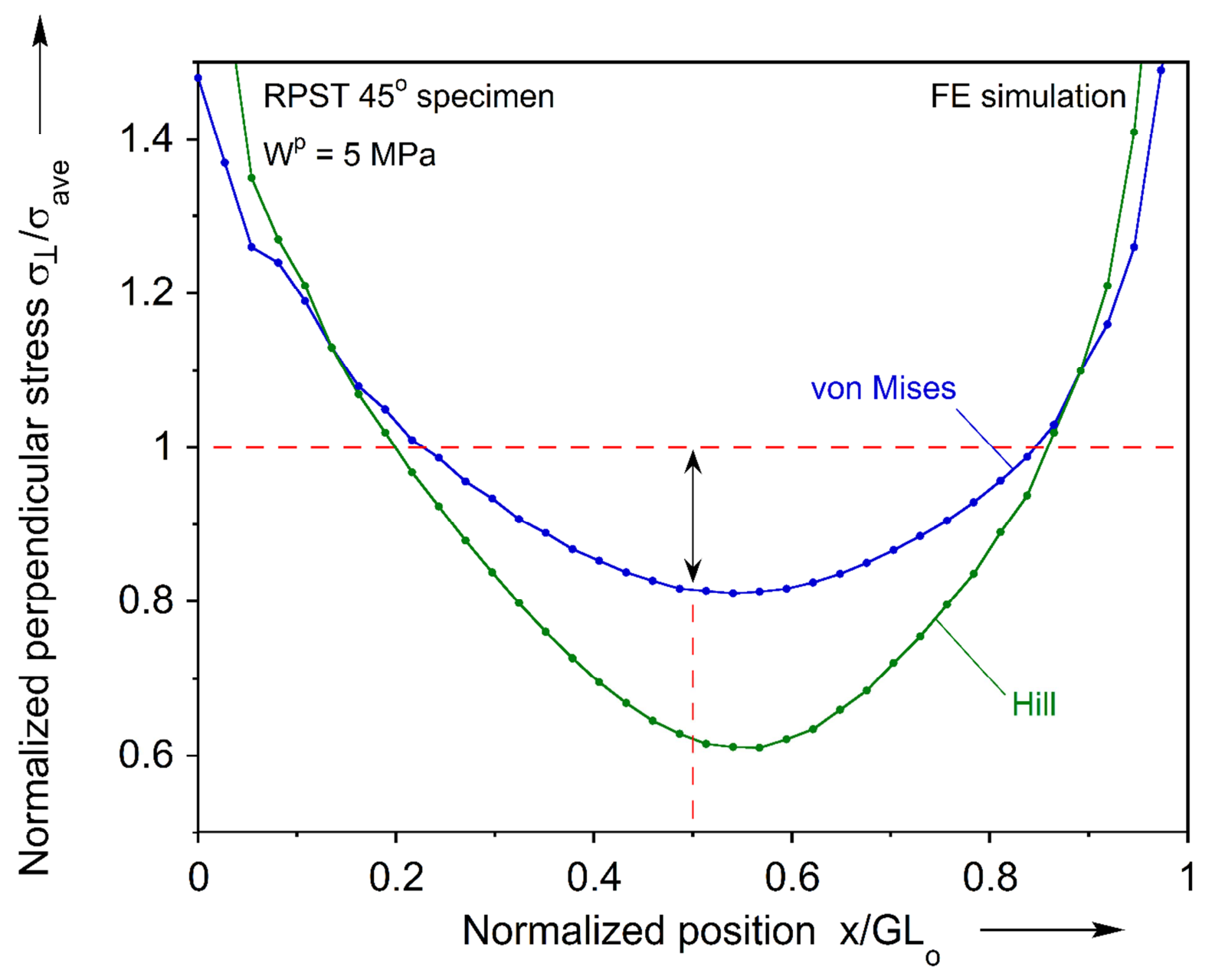

F19b 


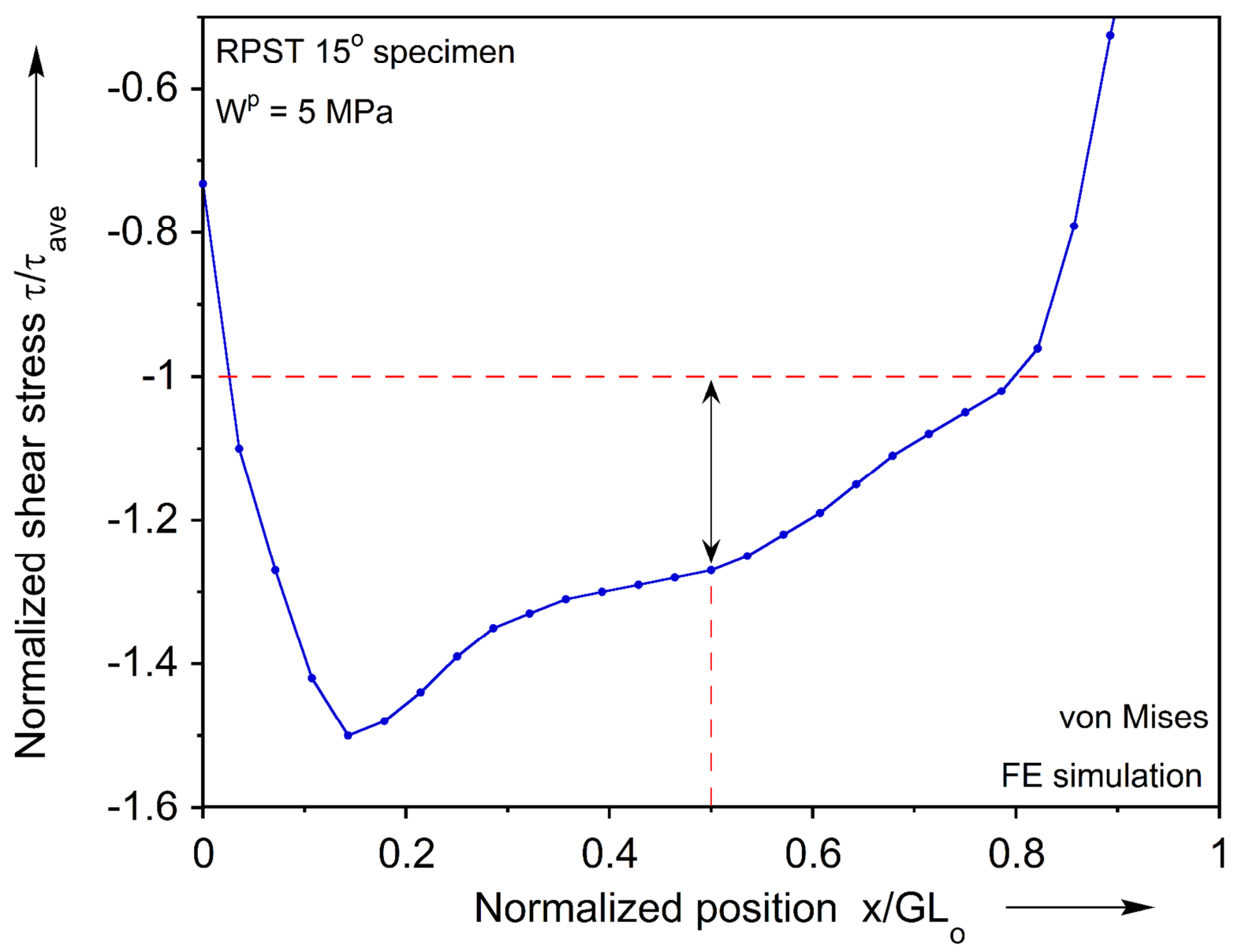

F20a 


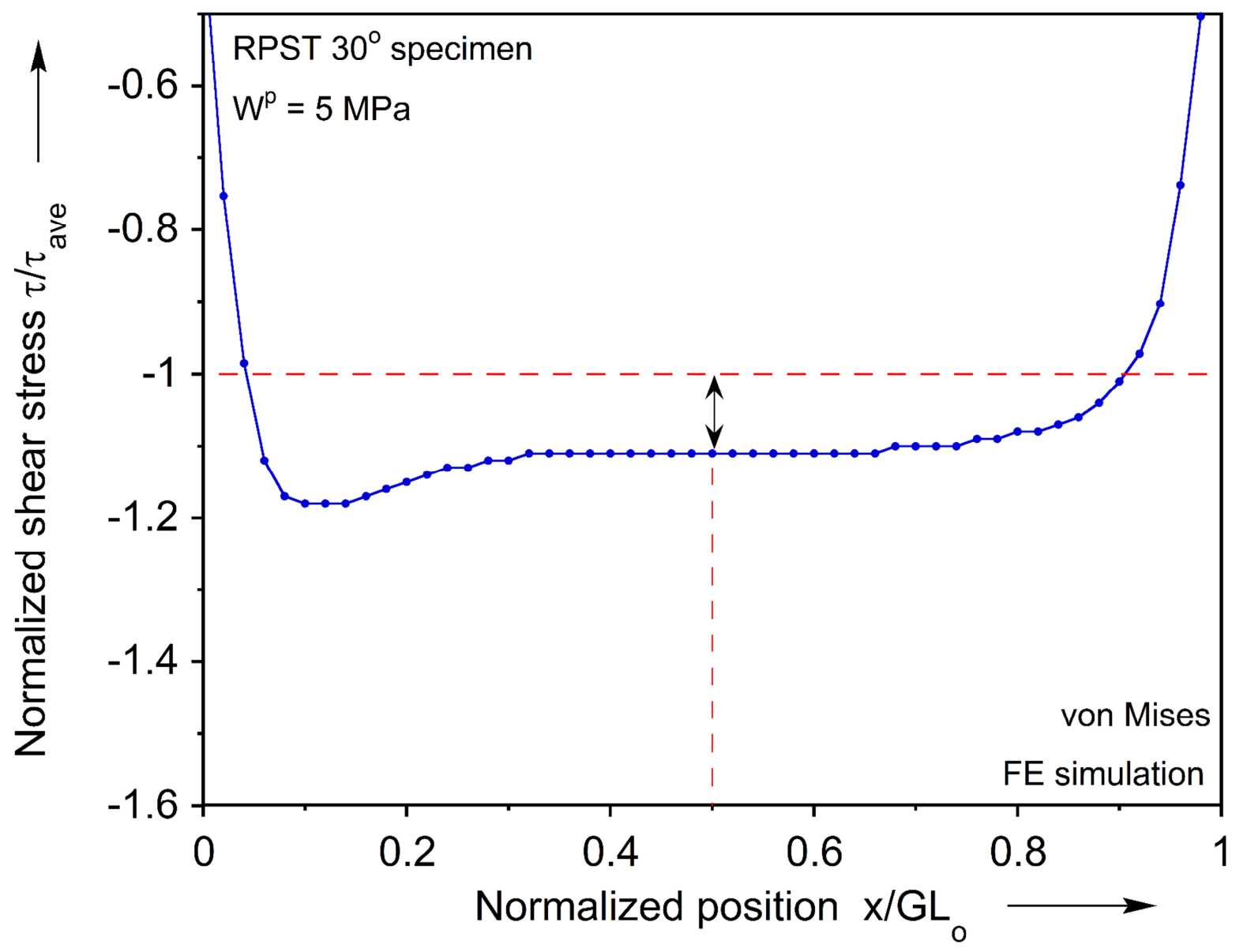

F20b 


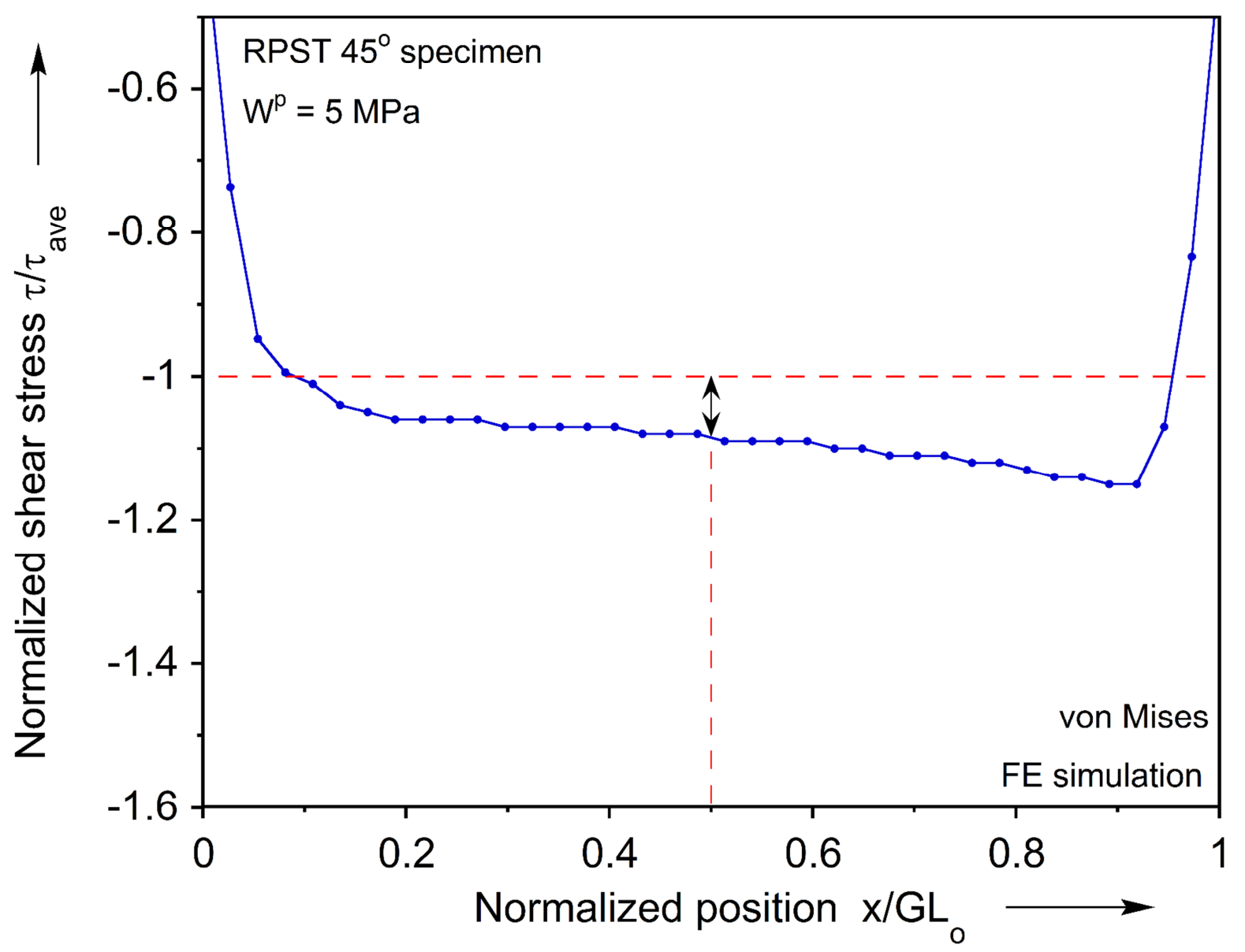

F20c 


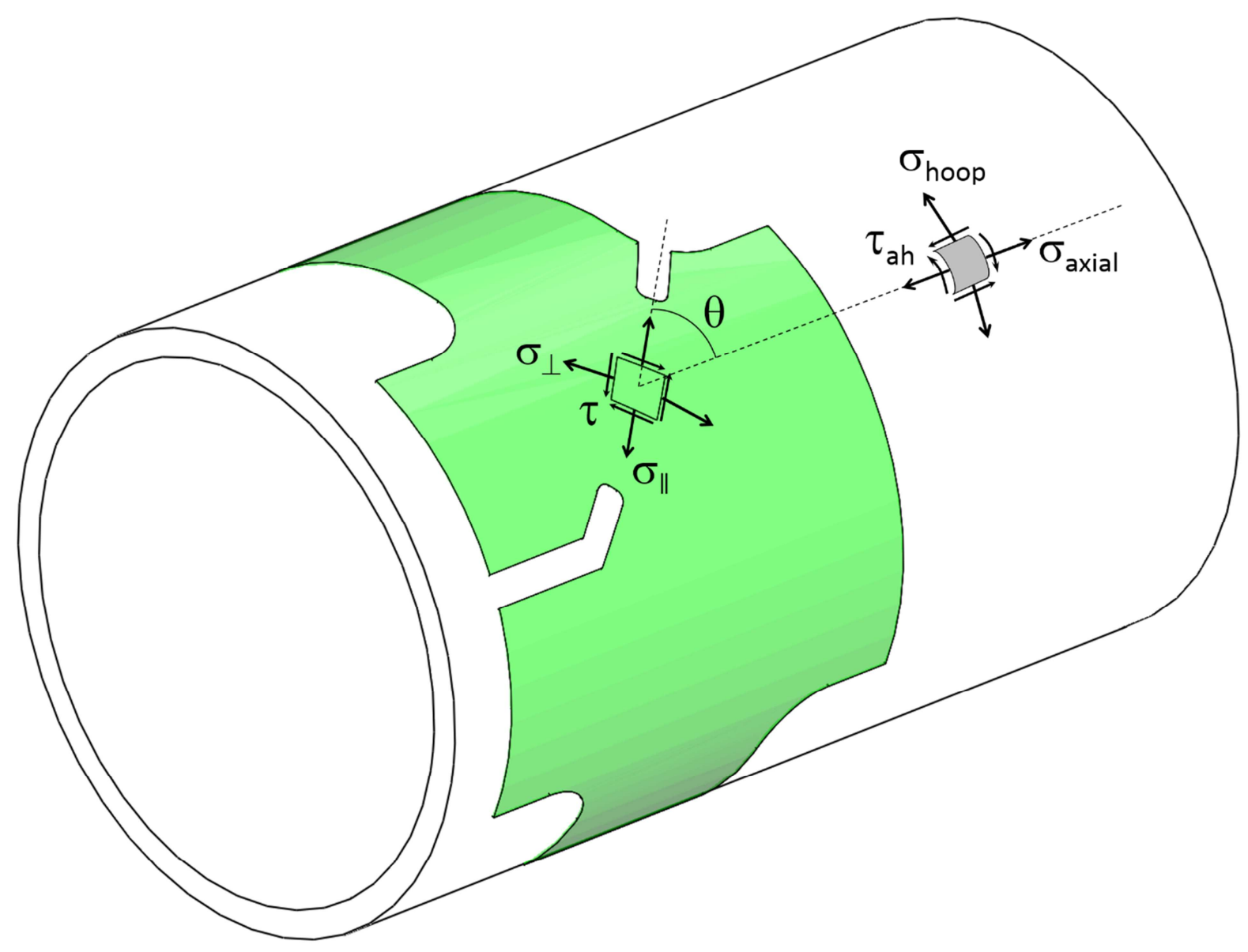

F21 


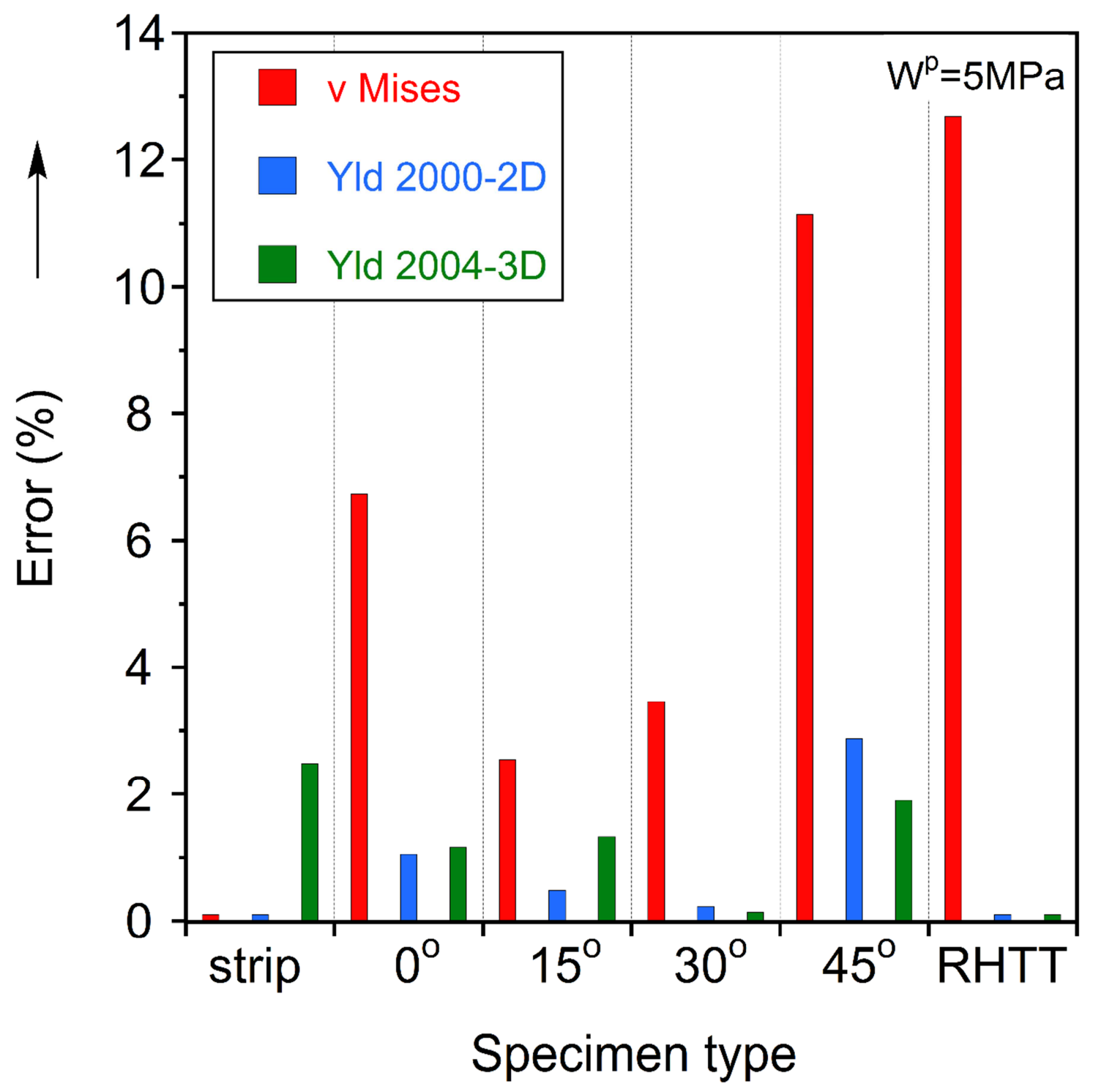

F22 


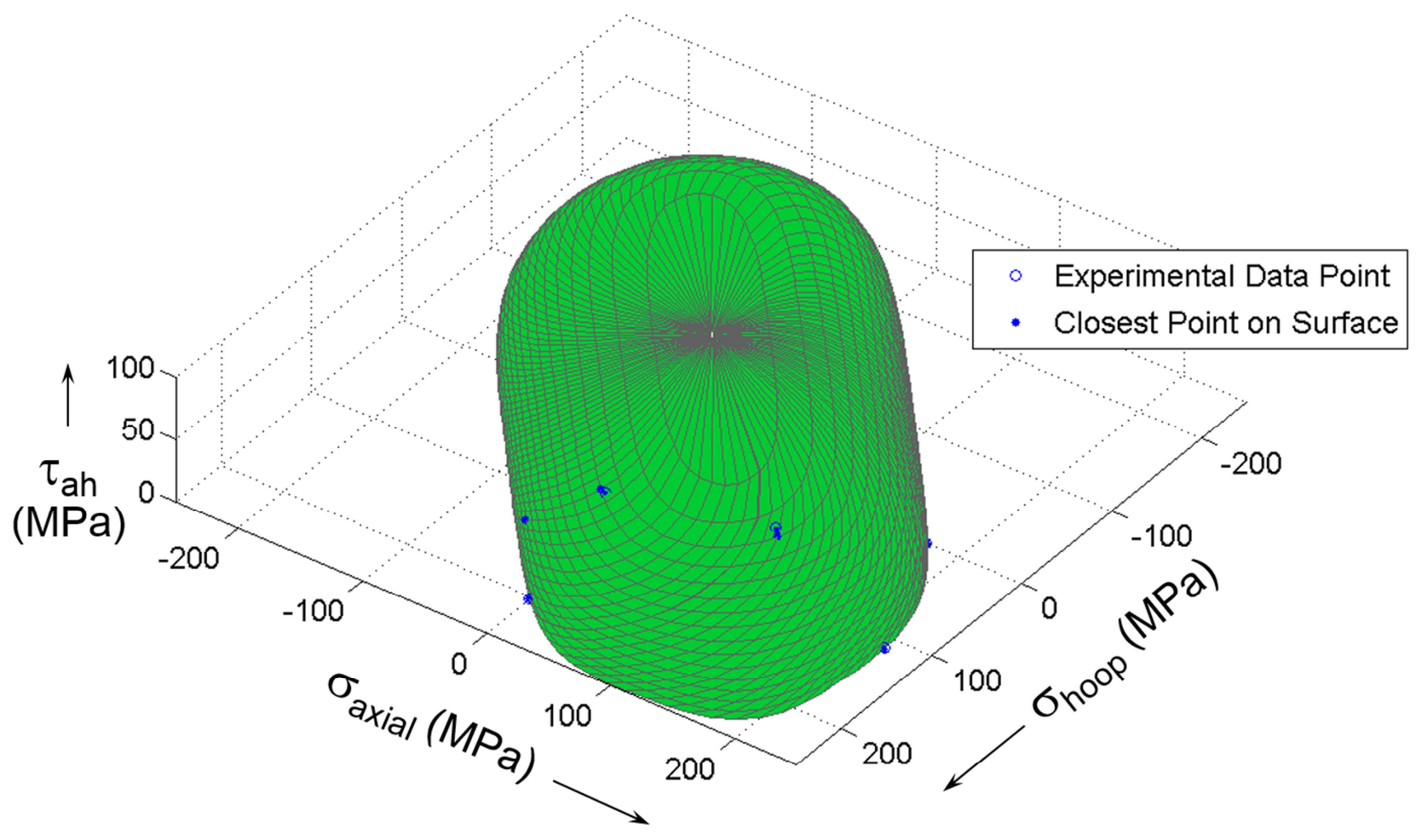

F23a 


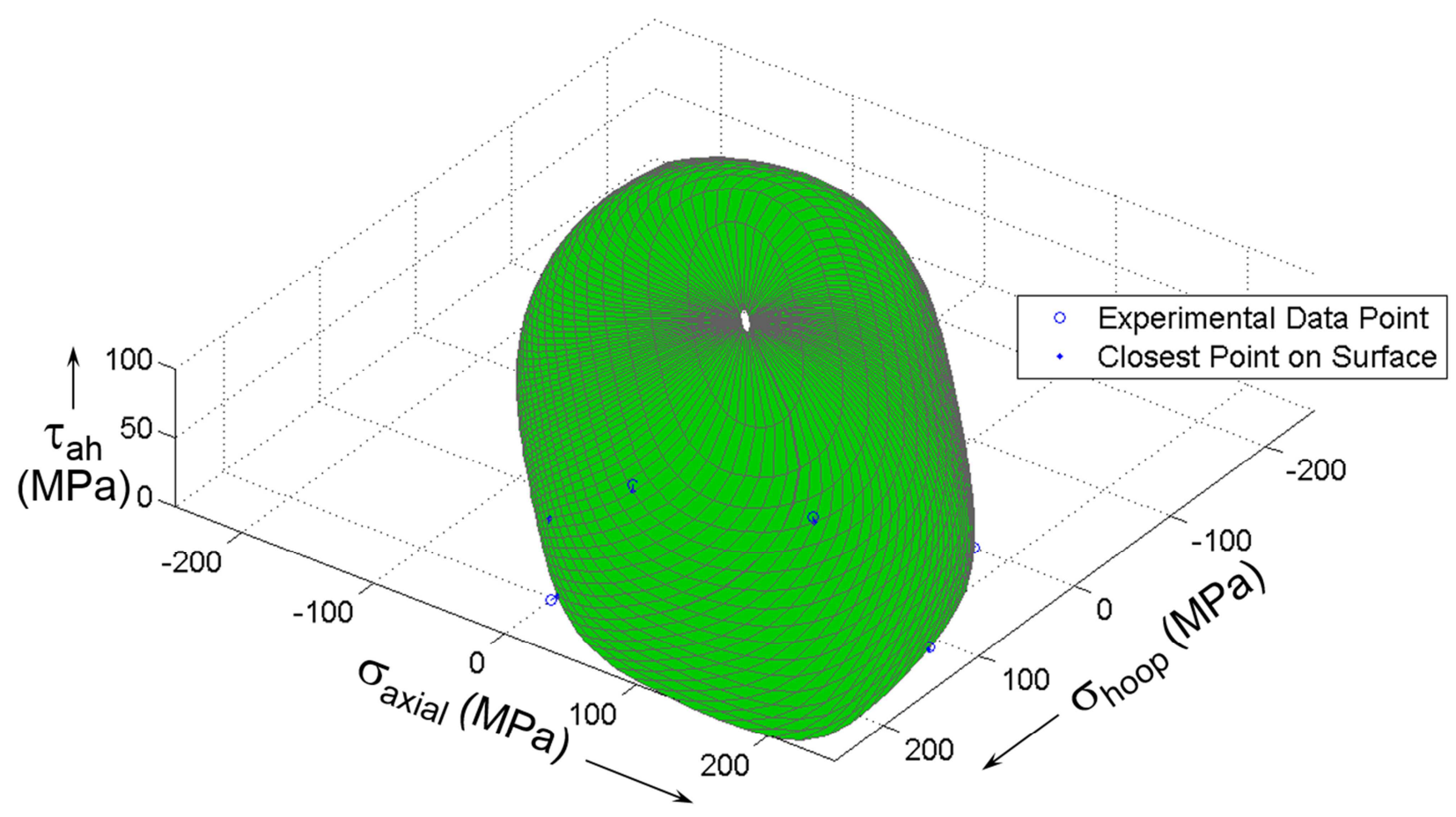

F23b 


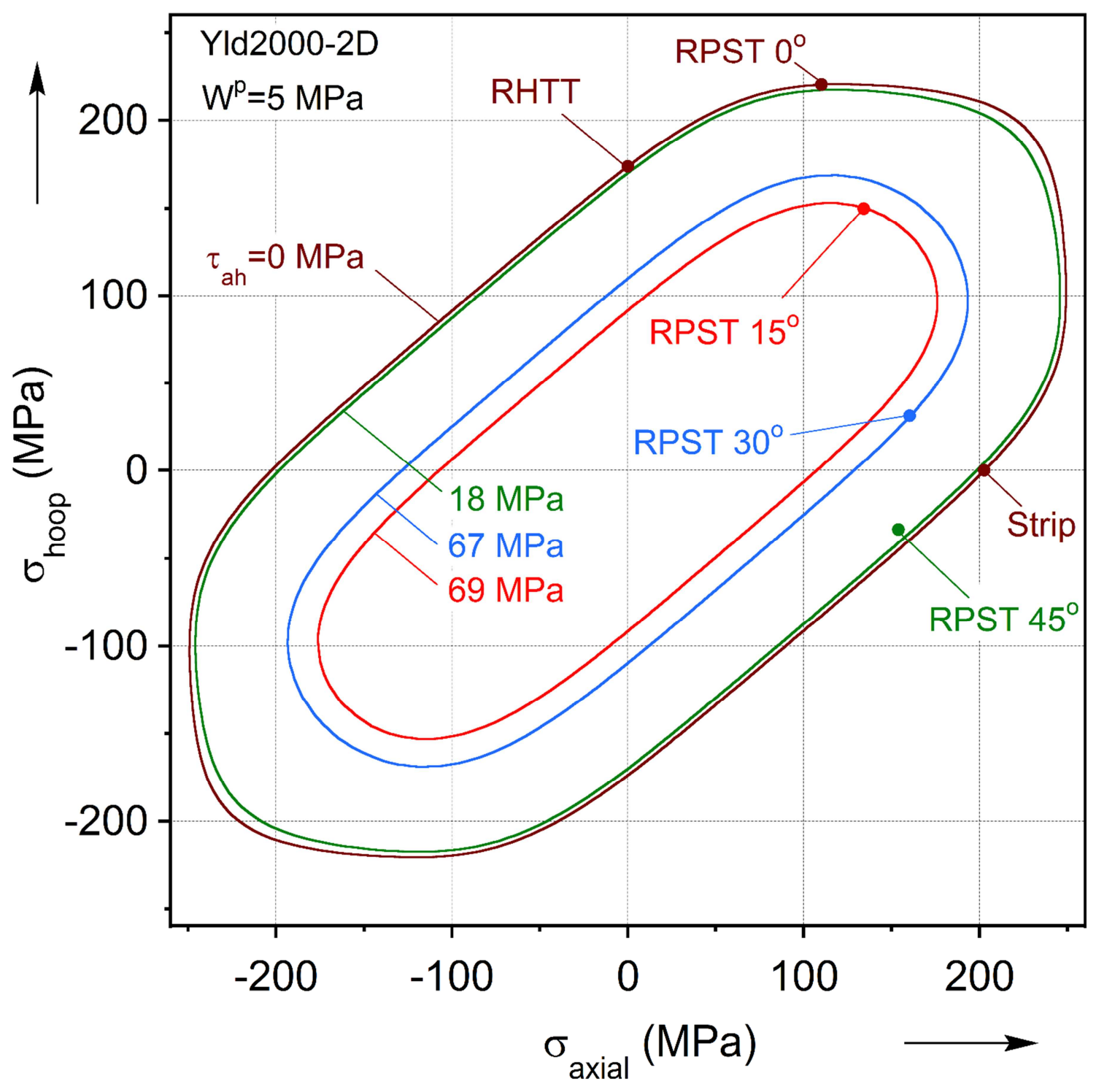

F24a 


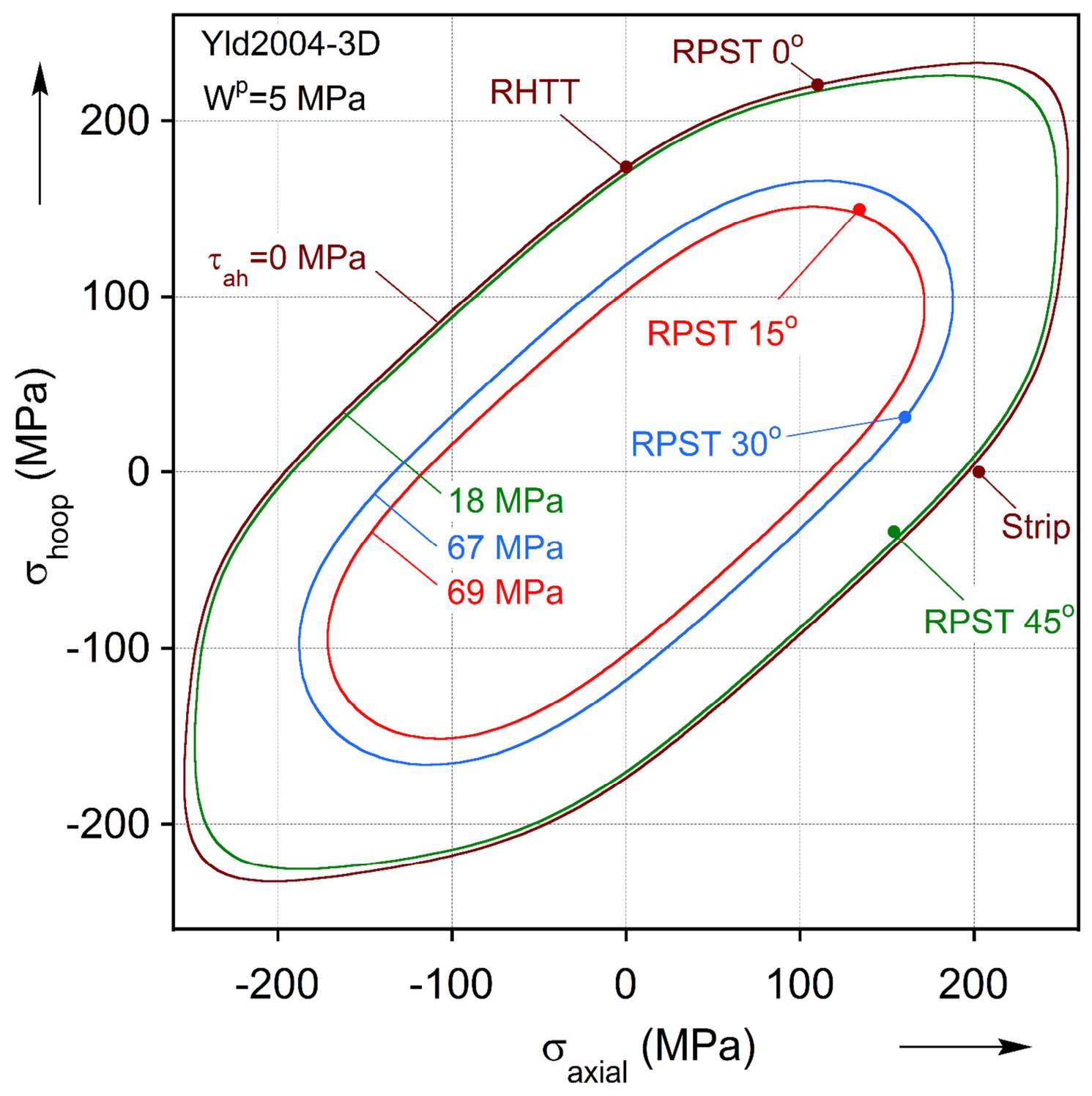

F24b 


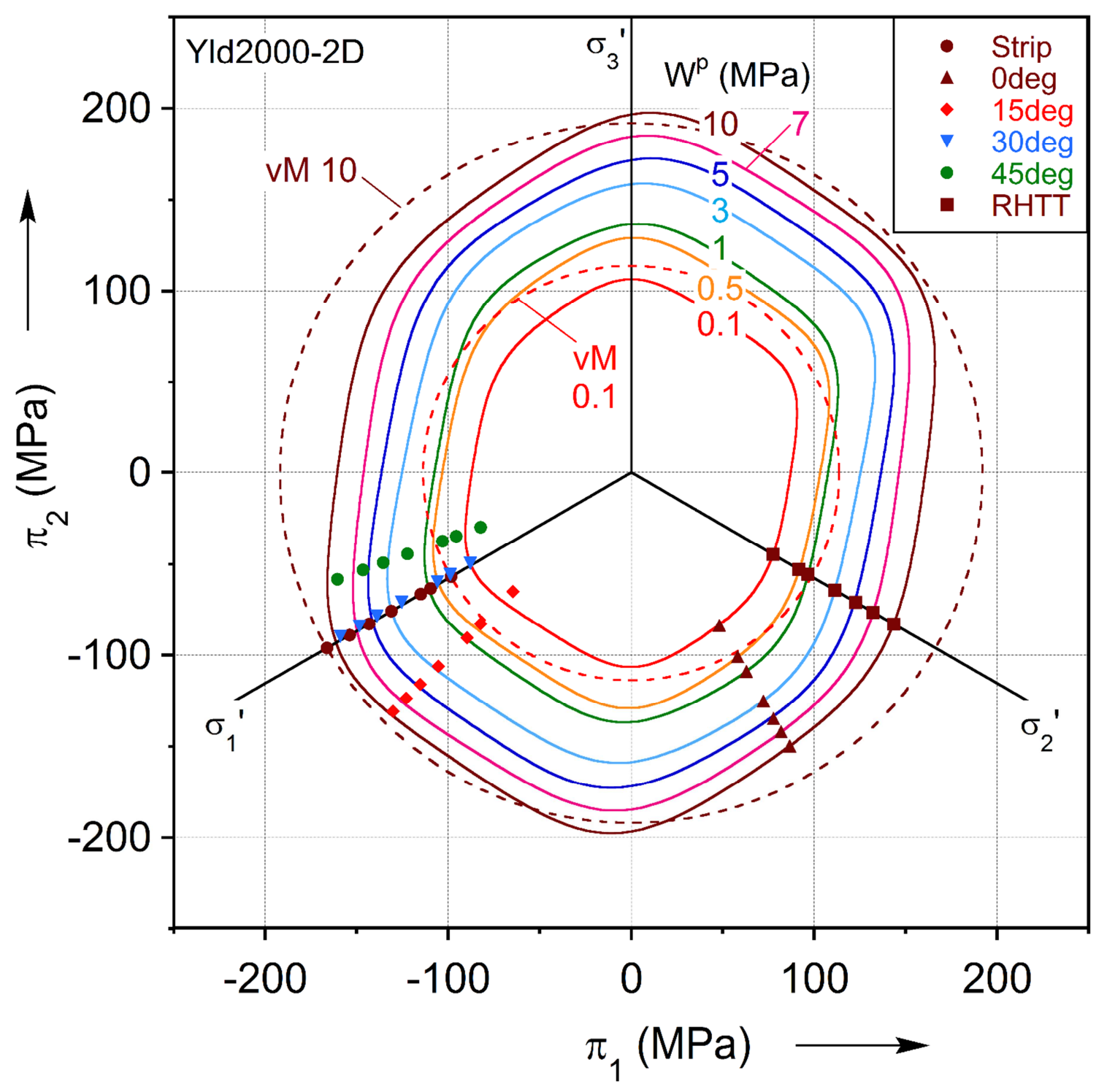

F25 

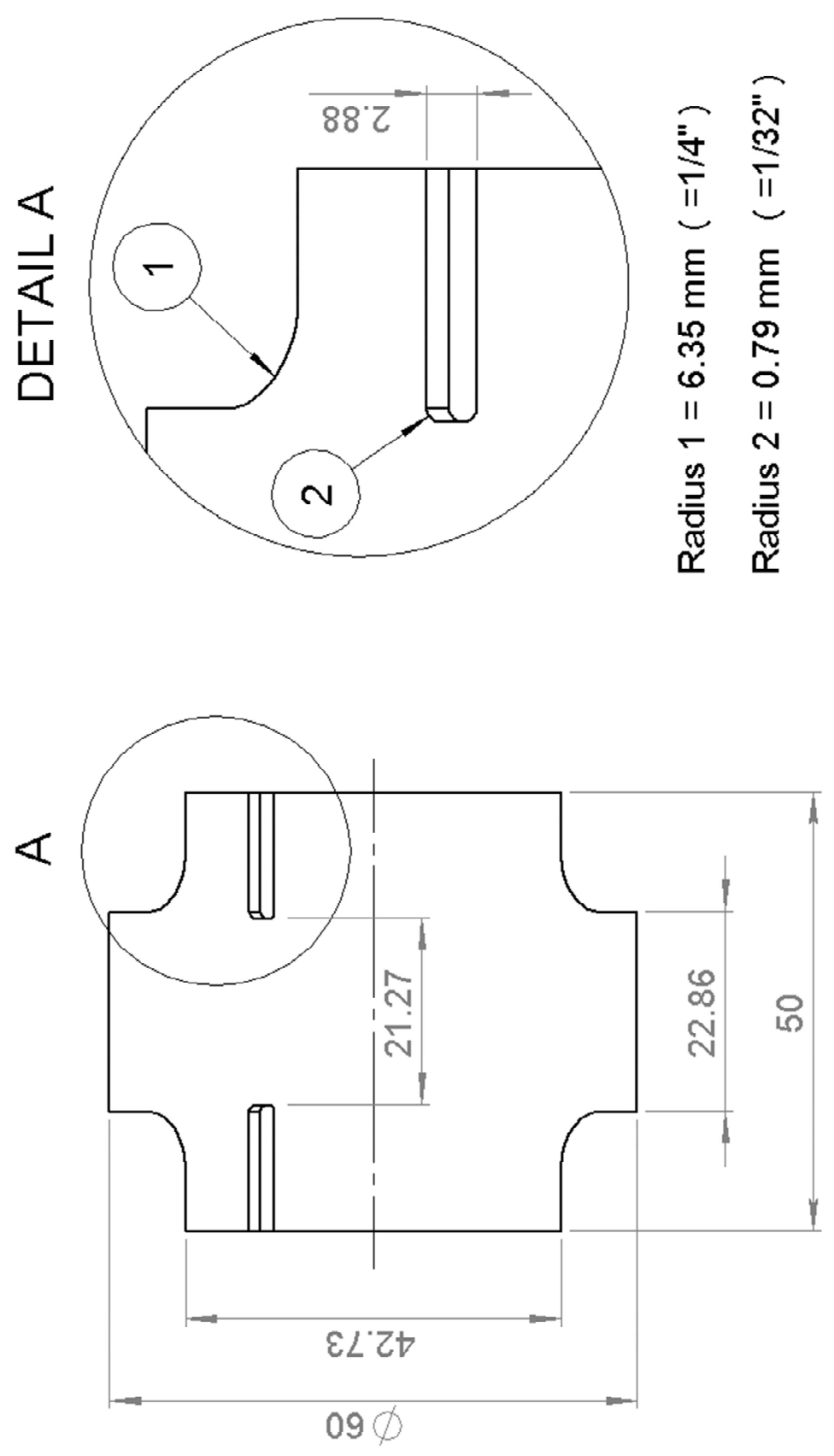

FA1 